\title{
Modeling and Simulation of Surfactant-Polymer Flooding using a New Hybrid Method
}

\author{
Prabir Daripa* and Sourav Dutta ${ }^{\dagger}$ \\ Department of Mathematics, Texas A\&M University \\ College Station, TX 77843-3368. \\ Dedicated to James Glimm \\ January 15, 2017
}

\begin{abstract}
Chemical enhanced oil recovery by surfactant-polymer (SP) flooding has been studied in two space dimensions. A new global pressure for incompressible, immiscible, multicomponent two-phase porous media flow has been derived in the context of SP flooding. This has been used to formulate a system of flow equations that incorporates the effect of capillary pressure and also the effect of polymer and surfactant on viscosity, interfacial tension and relative permeabilities of the two phases. The coupled system of equations for pressure, water saturation, polymer concentration and surfactant concentration has been solved using a new hybrid method in which the elliptic global pressure equation is solved using a discontinuous finite element method and the transport equations for water saturation and concentrations of the components are solved by a Modified Method $\underline{\text { Of }}$ Characteristics (MMOC) in the multicomponent setting. Numerical simulations have been performed to validate the method, both qualitatively and quantitatively, and to evaluate the relative performance of the various flooding schemes for several different heterogeneous reservoirs.
\end{abstract}

Keywords: surfactant-polymer flooding, global pressure, capillary pressure, finite element method, method of characteristics, numerical simulations, heterogeneous permeability

\section{Introduction}

One of the effective methods of tertiary oil recovery is Surfactant-Polymer (SP) flooding which involves injection of polymer and surfactant laden aqueous phase in oil reservoirs. Polymer in the displacing fluid improves oil recovery by inhibiting the growth rate of the fingering instability and by increasing the water saturation level behind the displacing front. See Daripa et al. [1] for a study of these phenomena during polymer flooding which in general is a less effective method of tertiary oil recovery than the SP flooding, a subject of present study. In the SP flooding, the use of surfactant further improves oil recovery by reducing the capillary pressure between the aqueous and the oil phases and by reducing the residual saturation limits of the rock matrix. ASP flooding in which Alkali is also used in the SP-laden aqueous phase, is supposed to be even more effective since the alkali produces surfactant in-situ thereby replenishing surfactant lost due to adsorption by the porous matrix during the transport of these chemical components by the flow through the porous matrix. In recent years, surfactant-polymer flooding has been shown to be preferable to even ASP flooding in highly heterogeneous reservoirs as the elimination of alkali removes undesirable inorganic scale

\footnotetext{
*Author for correspondence (email:daripa@math.tamu.edu)

†email:sdutta@math.tamu.edu
} 
formation, incompatibility with some polymers and demulsification difficulties $[2,3]$. Therefore, SP flooding is of significant current interest and is drawing a lot of attention $[4,5,6,7,8]$.

Multiphase flow in porous media is modeled mainly using two different approaches. The first approach is based on mass conservation laws for each of the fluids [9]. In the second approach, known as the fractional flow approach, these conservation laws are combined to obtain a new set of equations with the introduction of the total velocity, the global pressure and other ancillary functions. The global pressure was introduced by Chavent and Jaffré [10] for immiscible, incompressible two-phase flow without any components and was later revisited by several others (see for instance [11]). The fractional flow approach which is computationally more efficient than the two-pressure approach, has been adopted for numerical simulations in hydrology and petroleum reservoir problems. Recently the concept of global pressure has been extended to immiscible, compressible two-phase flows under the assumption of no mass transfer between the phases and taking into account the effects of gravity, capillary pressure and heterogeneity $[12,13]$. Some existence results for this new formulation have been presented [14] and its effectiveness in numerical simulations has been demonstrated [15]. This has been further extended to a global pressure formulation for the case of compositional, compressible two-phase flows with mass exchange between the phases [16]. A fully equivalent global pressure formulation for three-phase compressible flows has been established [17] and numerically validated [18]. In this paper, a global pressure formulation has been formally derived for immiscible, incompressible two-phase flows in the presence of dissolved components (polymer and surfactant). Unlike the two-phase flow models discussed above, the fractional flow formulation for multicomponent two-phase flow has a system of transport equations for the non-wetting phase saturation and the component concentrations which are coupled to the global pressure equation.

Classical finite difference and finite element methods often give rise to nonphysical oscillations in the numerical solution while classical upwind methods tend to generate excessive numerical diffusion that smears out the fronts. To avoid these issues, various high-order accurate finite difference methods $[1,19,20,21,22]$ and locally mass conservative finite volume methods $[23,24]$ have been developed for constructing numerical solutions of these multiphase multicomponent flows through heterogeneous porous media. Different types of finite element methods(FE) such as control volume FE (CVFE -[25, 26]), discontinuous Galerkin (DG -[27, 28, 29, 30]) and mixed FE [31, 32] methods have also become methods of choice for the reservoir simulation because these methods do not suffer from much grid orientation effects, allow local grid refinement and have high order of accuracy. These methods are also versatile and have been applied to other problems such as simulation of faults and fractures [33, 34, 35]. In the recent past, several multiscale FE and FV methods have also been proposed to better account for the variation in the reservoir properties over a large range of computational scales $[36,37,38]$. In problems where advection dominates diffusion, finite element methods have been found to exhibit excessive nonphysical oscillations for non-smooth solutions [39]. When standard upstream weighting approaches are applied to these FE methods to eliminate the nonphysical oscillations, they tend to smear out sharp fronts and suffer from grid orientation problems. While extremely fine mesh refinement can alleviate some of these problems, it has prohibitively high computational expense.

An alternative approach to solve time dependent advection-diffusion problems is given by the class of Eulerian-Lagrangian methods. In these methods, the advection part is treated in Lagrangian coordinates by propagating the solution along the characteristics for the advection part, while a Eulerian fixed mesh is used for the diffusion part [40,41]. In such a Lagrangian formulation, only the effect of the diffusion and the source terms are observed while the effect of the advection and/or the moving steep fronts are not seen. Hence, the solutions of the advection diffusion PDEs are much smoother along the characteristics than they are in the time direction and hence these methods usually allow larger time steps to be used in a numerical simulation while still maintaining its stability and accuracy. Also, these methods significantly reduce numerical diffusion and grid-orientation effects observed in upwind schemes, and are very competitive in terms of accuracy and efficiency [42, 43, 44].

The Modified Method $\underline{\text { Of }}$ Characteristics (MMOC) is one of the earliest methods (see [45]) in the class of Eulerian-Lagrangian methods to be widely accepted for numerical simulation of miscible and immiscible displacement problems [46]. Some of the significant subsequent improvements on this method include the modified method of characteristics with adjusted advection (MMOCAA, [42, 47]), the Eulerian-Lagrangian 
localized adjoint method (ELLAM, [48]), the characteristic mixed finite element method (CMFEM, [49]), the locally conservative Eulerian-Lagrangian method (LCELM, [50]), the characteristic finite volume element method (CFVEM, [51]) and the Eulerian-Lagrangian discontinuous Galerkin method (ELDG, [52]). The MMOCAA is a mass-conserving time discretization procedure which inherits the competitiveness of the MMOC. The conservative modified method of characteristics (CMMOC, [53]) is a variant of the MMOCAA which adjusts the convection implicitly and minimizes the error of the mass balance problem. The ELLAM and CMFEM are some of the other variants of the characteristics based method which also conserve mass. So far, the MMOC and its variants have only been studied in the context of miscible and immiscible, purely two-phase flow problems that lead to a system of coupled equations for solving one of the phase saturations and one of the phase pressures. In this paper, the MMOC approach has been applied to solve the system of transport equations arising in the context of multicomponent two-phase flows with one phase saturation and two component concentrations as the primary transport variables. Towards this end, it is worth mentioning here that the front tracking method has been applied to solve a similar system as ours but of less complexity when the surfactant is not present and the effect of capillary pressure is neglected. We direct the readers to Daripa et al. [20] and the papers cited therein.

In this paper, we propose a model that describes the displacement processes during the SP flooding in the form of a new global pressure formulation. The model is numerically solved using a hybrid methodology that uses a non-traditional discontinuous finite element method for the global pressure, in combination with the modified method of characteristics (MMOC) and time implicit methods to solve the system of the advectiondiffusion equations for the phase saturations and the concentrations of various chemical components. To validate and demonstrate the accuracy and performance of the method, we use numerical simulations of waterflooding and polymer flooding in different heterogeneous permeability fields and flow geometries. Then, results from numerical simulation studies comparing various flooding schemes for the SP flooding in heterogeneous permeability fields are presented. In a sequel which is in progress, we prove convergence of this method.

This paper is laid out as follows. In section 2, the global pressure formulation of the governing equations for SP flooding is derived and presented along with the various models of relative permeability and their dependence on the water saturation and the concentrations of the polymer and the surfactant. In section 3 , we present the numerical method: the computational grids, the non-traditional discontinuous finite element method for the pressure equation, the MMOC for the transport equations of the SP flooding, the construction of multiscale heterogeneity, and the computational algorithm along with a pseudocode and a flowchart. We present and discuss the numerical results in section 4 . Finally we conclude in section 5.

\section{Mathematical model: Surfactant-polymer flood}

SP flooding is the process where the reservoir is flooded with the SP laden aqueous solution. This new aqueous phase displaces the oil during this flooding process. This can achieve a higher level of oil recovery than the polymer flood and become more economical if an appropriate combination of surfactant and polymer is used. Polymer is used to increase the viscosity of the displacing aqueous phase, which in turn stabilizes the oil-water interface and partially inhibits the growth of unwanted finger patterns. Surfactants have at least one hydrophobic group (non-polar tail) and one hydrophilic group (polar head) in the same molecule, as shown in Fig. 1(a). This dual character allows them to sufficiently lower the interfacial tension and also remarkably alter the wetting properties. These factors contribute towards lowering the capillary pressure barrier and increasing the relative permeability of the rock, thereby improving oil recovery. However, the dual character of the surfactant also causes the spontaneous formation of clusters known as micelles. These micelles may interact with the polymer chains to form more complicated aggregates. Depending on the $\mathrm{pH}$ level of the system, the polymer can sometimes be lost due to adsorption on the rock surface. Moreover, the thin capillary structure of the rock pores causes the polymer chains to stretch or coil, thus changing the nature of the SP laden aqueous phase. Some of these effects are illustrated in Fig. 1(b). For the purpose of the current work, some simplifying assumptions have been made that enable us to study the impact of the 


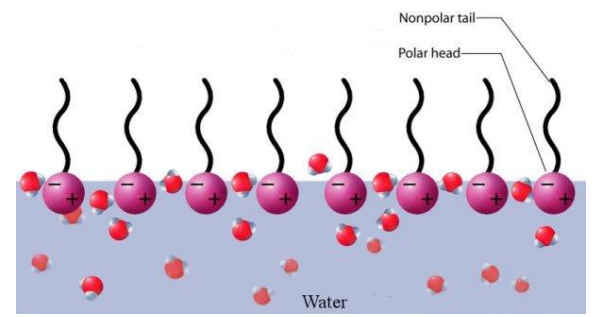

(a) Surfactant molecules arranged on an interface

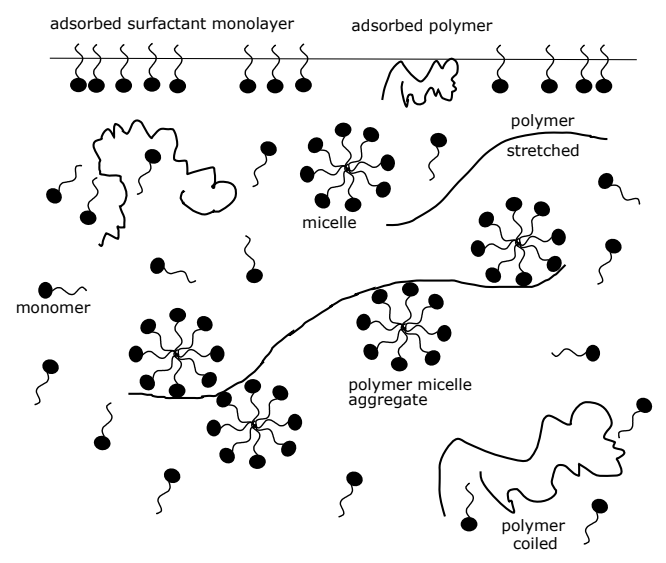

(b) Dynamics of a surfactant polymer solution

Figure 1: Pictorial representation of the rheology and behavior of surfactant molecules (a) near an interface and (b) in a solution containing dissolved polymer. The left diagram has been taken from the Wikipedia link - https://commons.wikimedia.org/wiki/File:Surfactant.jpg

components on the aqueous phase viscosity, interfacial tension and capillary pressure.

SP flooding is modeled here as an incompressible, immiscible, multicomponent two-phase flow through porous media. Let $\Omega$ be a finite domain representing the porous reservoir with boundary $\partial \Omega$. Let the non-wetting phase (oil) and the wetting phase (water or an aqueous solution of polymer and surfactant) be denoted by the subscripts " $o$ " and " $a$ " respectively. Let $s_{j}$ denote the saturation (volume fraction), $\mathbf{v}_{j}$ the velocity, $p_{j}$ the pressure, $q_{j}$ the appropriate source/sink term, $k_{r j}$ the relative permeability, $\mu_{j}$ the viscosity and $\lambda_{j}=k_{r j} / \mu_{j}$ denote the mobility of phase $j$ where $j=o$ or $a$. Also, let $\mathbf{K}(\mathbf{x})$ be the absolute permeability tensor, $\phi$ be the porosity of the medium, $\lambda=\lambda_{a}+\lambda_{o}$ be the total mobility and $\mathbf{v}=\mathbf{v}_{a}+\mathbf{v}_{o}$ be the total velocity. Then considering the hypothesis of capillary pressure and relative permeability we can write the following equations arising from the conservation of mass of each phase

$$
\begin{array}{ll}
\phi \frac{\partial s_{a}}{\partial t}+\nabla \cdot \mathbf{v}_{a}=q_{a}, & (\mathbf{x}, t) \in \Omega \times(0, T], \\
\phi \frac{\partial s_{o}}{\partial t}+\nabla \cdot \mathbf{v}_{o}=q_{o}, & (\mathbf{x}, t) \in \Omega \times(0, T] .
\end{array}
$$

The equations for conservation of mass of the polymer and the surfactant dissolved in the aqueous phase are given by

$$
\begin{array}{cc}
\phi \frac{\partial\left(c s_{a}\right)}{\partial t}+\nabla \cdot\left(c \mathbf{v}_{a}\right)=c^{i} q_{a} \delta\left(\mathbf{x}-\mathbf{x}^{i}\right)+c q_{a} \delta\left(\mathbf{x}-\mathbf{x}^{p}\right), & (\mathbf{x}, t) \in \Omega \times(0, T], \\
\phi \frac{\partial\left(\Gamma s_{a}\right)}{\partial t}+\nabla \cdot\left(\Gamma \mathbf{v}_{a}\right)=\Gamma^{i} q_{a} \delta\left(\mathbf{x}-\mathbf{x}^{i}\right)+\Gamma q_{a} \delta\left(\mathbf{x}-\mathbf{x}^{p}\right), & (\mathbf{x}, t) \in \Omega \times(0, T],
\end{array}
$$

where $c$ is the concentration (volume fraction in the aqueous phase) of the dissolved polymer and $\Gamma$ is the concentration of the surfactant while $c^{i}$ and $\Gamma^{i}$ are the concentrations of polymer and surfactant in the injected fluid, respectively. It is assumed that both the components are getting only passively advected with negligible diffusion and no adsorption losses. Also, $\mathbf{x}^{i}$ is the location of the injection well and $\mathbf{x}^{p}$ is the location of the production well, both of which are treated as point source/sink terms and $\delta$ is the Dirac-delta 
function. The conservation of momentum is given by the Darcy-Muskat law for each phase

$$
\begin{array}{ll}
\mathbf{v}_{a}=-\mathbf{K}(\mathbf{x}) \lambda_{a} \nabla p_{a}, & \mathbf{x} \in \Omega, \\
\mathbf{v}_{o}=-\mathbf{K}(\mathbf{x}) \lambda_{o} \nabla p_{o}, & \mathbf{x} \in \Omega .
\end{array}
$$

We consider the porous medium to be initially saturated with the two phases and hence we have

$$
s_{o}+s_{a}=1 \text {. }
$$

Henceforth we use the notation $s$ for $s_{a}$. The capillary pressure, $p_{c}$, is given by

$$
p_{c}(s)=p_{o}-p_{a} .
$$

The following initial and boundary conditions are prescribed.

$$
\begin{array}{rllll}
s(\mathbf{x})=s_{0}(\mathbf{x}), & c(\mathbf{x})=c_{0}(\mathbf{x}), & \Gamma(\mathbf{x})=\Gamma_{0}(\mathbf{x}) ; & \mathbf{x} \in \Omega, & t=0, \\
\boldsymbol{\nabla} s \cdot \hat{\mathbf{n}}=0, & \boldsymbol{\nabla} c \cdot \hat{\mathbf{n}}=0, & \boldsymbol{\nabla} \Gamma \cdot \hat{\mathbf{n}}=0 \quad \& \quad \mathbf{v}_{j} \cdot \hat{\mathbf{n}}=0 ; & \mathbf{x} \in \partial \Omega, & t>0, \quad(j=a, o),
\end{array}
$$

where $\hat{\mathbf{n}}$ is a unit vector normal to $\partial \Omega$. The source/sink terms in eqs. (1a) and (1b) are given by

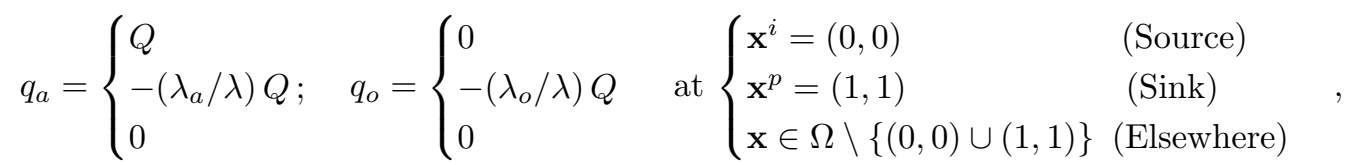

where $Q$ is the volumetric injection/production rate. In the above, we have made the physically relevant assumption that oil is never injected and the fluid mixture obtained at the production well is proportional to the resident fluid at that point.

Above equations describe the flow and transport problem in the phase pressure formulation, which is a system of strongly coupled non-linear equations. This system is also degenerate because only eq. (4b) (eq. (4a)) will survive at $s=0(1)$ where the phase pressure $p_{a}\left(p_{o}\right)$ has no meaning. For these and many other reasons, mathematical and numerical studies of this problem including numerical simulations are very challenging. In order to avoid this difficulty, we propose a new global pressure formulation for incompressible, immiscible two-phase flows with dissolved components. A formal derivation is given below in brief.

We observe the following identity

$$
\mathbf{v}_{a}=\frac{\lambda_{o}}{\lambda} \mathbf{v}_{a}-\frac{\lambda_{a}}{\lambda} \mathbf{v}_{o}+\frac{\lambda_{a}}{\lambda} \mathbf{v}
$$

Substituting eq. (9) in eq. (1a) and then using eq. (4a) and eq. (4b), we obtain

$$
\phi \frac{\partial s}{\partial t}-\boldsymbol{\nabla} \cdot\left\{\mathbf{K}(\mathbf{x}) \frac{\lambda_{o}}{\lambda} \lambda_{a} \boldsymbol{\nabla} p_{a}-\mathbf{K}(\mathbf{x}) \frac{\lambda_{a}}{\lambda} \lambda_{o} \nabla p_{o}\right\}+\nabla \cdot\left\{\frac{\lambda_{a}}{\lambda} \mathbf{v}\right\}=q_{a},
$$

or equivalently,

$$
\phi \frac{\partial s}{\partial t}+\boldsymbol{\nabla} \cdot\left\{\mathbf{K}(\mathbf{x}) f_{a}(s, c, \Gamma) \lambda_{o} \boldsymbol{\nabla}\left(p_{c}(s, \Gamma)\right)\right\}+\boldsymbol{\nabla} \cdot\left(f_{a}(s, c, \Gamma) \mathbf{v}\right)=q_{a} .
$$

Here $f_{j}(s, c, \Gamma)=\lambda_{j}(s, c, \Gamma) / \lambda(s, c, \Gamma)$ is the fractional flow function of the phase $j$. Equation (10) is called the saturation equation. For purposes below, we define

$$
\begin{aligned}
\eta^{c}(s, c, \Gamma) & =\int_{s_{c}}^{s} \frac{\partial}{\partial c}\left(f_{o}-f_{a}\right)(\zeta, c, \Gamma) \frac{d p_{c}}{d \zeta}(\zeta, \Gamma) d \zeta+\int_{\Gamma_{c}}^{\Gamma} \frac{\partial}{\partial c}\left(f_{o}-f_{a}\right)(s, c, \xi) \frac{d p_{c}}{d \xi}(s, \xi) d \xi, \\
\eta^{s}(s, c, \Gamma) & =\int_{\Gamma_{c}}^{\Gamma} \frac{\partial}{\partial s}\left(f_{o}-f_{a}\right)(s, c, \xi) \frac{d p_{c}}{d \xi}(s, \xi) d \xi \\
\eta^{\Gamma}(s, c, \Gamma) & =\int_{s_{c}}^{s} \frac{\partial}{\partial \Gamma}\left(f_{o}-f_{a}\right)(\zeta, c, \Gamma) \frac{d p_{c}}{d \zeta}(\zeta, \Gamma) d \zeta,
\end{aligned}
$$


where $s_{c}$ is the value of the aqueous phase saturation at which $p_{c}\left(s_{c}, \Gamma\right)=0$ and $\Gamma_{c}$ is the surfactant concentration value defined similarly by $p_{c}\left(s, \Gamma_{c}\right)=0$. Using the above relations, we define a function $p$, called the global pressure, as follows.

$$
\begin{aligned}
p=\frac{1}{2}\left(p_{o}+p_{a}\right) & +\frac{1}{2} \int_{s_{c}}^{s}\left(f_{o}-f_{a}\right)(\zeta, c, \Gamma) \frac{d p_{c}}{d \zeta}(\zeta, \Gamma) d \zeta+\frac{1}{2} \int_{\Gamma_{c}}^{\Gamma}\left(f_{o}-f_{a}\right)(s, c, \xi) \frac{d p_{c}}{d \xi}(s, \xi) d \xi \\
& -\frac{1}{2} \int \eta^{c}(s, c, \Gamma)\left(\frac{\partial c}{\partial x} d x+\frac{\partial c}{\partial y} d y\right)-\frac{1}{2} \int \eta^{s}(s, c, \Gamma)\left(\frac{\partial s}{\partial x} d x+\frac{\partial s}{\partial y} d y\right) \\
& -\frac{1}{2} \int \eta^{\Gamma}(s, c, \Gamma)\left(\frac{\partial \Gamma}{\partial x} d x+\frac{\partial \Gamma}{\partial y} d y\right)+C,
\end{aligned}
$$

where $C$ is a reference pressure which takes the place of an integration constant in the calculations. Taking gradients on both sides of eq. (12) and simplifying we obtain

$$
\boldsymbol{\nabla} p=\frac{1}{2} \boldsymbol{\nabla}\left(p_{o}+p_{a}\right)+\frac{1}{2}\left(f_{o}-f_{a}\right)(s, c, \Gamma) \nabla p_{c}(s, \Gamma) .
$$

In the absence of the polymer and the surfactant, eq. (12) reduces to the global pressure function for incompressible, immiscible two-phase flows (see [10]). Multiplying both sides of eq. (13) with $\mathbf{K}(\mathbf{x}) \lambda$ and simplifying we get

$$
-\mathbf{K}(\mathbf{x}) \lambda \boldsymbol{\nabla} p=\mathbf{v} .
$$

Due to the incompressibility of the phases, we have

$$
\boldsymbol{\nabla} \cdot \mathbf{v}=q_{a}+q_{o} .
$$

Using eq. (14) in eq. (15) we finally get

$$
-\boldsymbol{\nabla} \cdot(\mathbf{K}(\mathbf{x}) \lambda(s, c, \Gamma) \nabla p)=q_{a}+q_{o} .
$$

Equation (16) is the global pressure equation. Solving this equation (see below) yields the global pressure $p$ from which the phase pressures $p_{a}$ and $p_{o}$ are easily recovered using the definition (6) for the capillary pressure and the definition (12) for the global pressure. In this formal derivation of the global pressure variable, we have only assumed the following physically realistic functional dependencies, $f_{a}=f_{a}(s, c, \Gamma)$, $f_{o}=f_{o}(s, c, \Gamma)$ and $p_{c}=p_{c}(s, \Gamma)$ and not specific functional forms. However, a similar procedure can be adopted to derive a more general form of global pressure for any multicomponent two-phase flow system.

Hence the new system of equations for the SP model in non-conservative form is given by

$$
\begin{aligned}
& -\boldsymbol{\nabla} \cdot(\mathbf{K}(\mathbf{x}) \lambda(s, c, \Gamma) \boldsymbol{\nabla} p)=q_{a}+q_{o}, \\
& \phi \frac{\partial s}{\partial t}+\frac{\partial f_{a}}{\partial s} \mathbf{v} \cdot \boldsymbol{\nabla} s+\boldsymbol{\nabla} \cdot\left(D \frac{\partial p_{c}}{\partial s} \boldsymbol{\nabla} s\right)=g_{s}-\frac{\partial f_{a}}{\partial c} \mathbf{v} \cdot \boldsymbol{\nabla} c-\frac{\partial f_{a}}{\partial \Gamma} \mathbf{v} \cdot \nabla \Gamma-\nabla \cdot\left(D \frac{\partial p_{c}}{\partial \Gamma} \boldsymbol{\nabla} \Gamma\right), \\
& \phi \frac{\partial c}{\partial t}+\left(\frac{f_{a}}{s} \mathbf{v}+\frac{D}{s} \frac{\partial p_{c}}{\partial s} \boldsymbol{\nabla} s+\frac{D}{s} \frac{\partial p_{c}}{\partial \Gamma} \boldsymbol{\nabla} \Gamma\right) \cdot \boldsymbol{\nabla} c=g_{c}, \\
& \phi \frac{\partial \Gamma}{\partial t}+\left(\frac{f_{a}}{s} \mathbf{v}+\frac{D}{s} \frac{\partial p_{c}}{\partial s} \boldsymbol{\nabla} s+\frac{D}{s} \frac{\partial p_{c}}{\partial \Gamma} \boldsymbol{\nabla} \Gamma\right) \cdot \boldsymbol{\nabla} \Gamma=g_{\Gamma},
\end{aligned}
$$

where $D(s, c, \Gamma)=\mathbf{K}(\mathbf{x}) \lambda_{o}(s, \Gamma) f_{a}(s, c, \Gamma)$ and

$$
g_{s}=\left\{\begin{array}{ll}
\left(1-f_{a}\right) Q & g_{c}= \begin{cases}\left(c^{i}-c\right) Q / s \\
0\end{cases}
\end{array} g_{\Gamma}=\left\{\begin{array}{ll}
\left(\Gamma^{i}-\Gamma\right) Q / s \\
0
\end{array} \text { at } \mathbf{x}= \begin{cases}\mathbf{x}^{i} & \text { (source) } \\
\Omega \backslash\left\{\mathbf{x}^{i}\right\} & \text { (elsewhere) }\end{cases}\right.\right.
$$


In this paper, potential degeneracies in the capillary term of eq. (17b) has been avoided in the numerical simulations. Please refer to section 3.2 for a more detailed discussion. In order to solve the system of eqs. (17a)-(17d), we need the viscosity relations, and the constitutive relations for the capillary pressure and the relative permeabilities. These relations are given below.

The aqueous phase viscosity is modeled by a linear function of polymer concentration.

$$
\mu_{a}=\mu_{w}(1+\gamma c)
$$

Here $\mu_{w}$ is the viscosity of pure water and the coefficient $\gamma$ characterizes the particular polymer. In the numerical simulations, we vary the viscosity ratio $M_{\mu}=\mu_{o} / \mu_{w}$ for waterflooding and $M_{\mu}=\mu_{o} / \mu_{a}$ for polymer flooding between the values of 1.25 and 10 .

Capillary pressure is strongly dependent on the surfactant concentration. It reduces steadily with increasing surfactant concentration up to the CMC (Critical Micelle Concentration) due to the reduction in interfacial tension (IFT). The relationship of IFT with surfactant concentration has been extensively studied experimentally and is known to be inversely proportional (Fig. 2). In order to model this complex dependence of capillary pressure on $s$ as well as on $\Gamma$, we adopt the approach (see [54]) that is often followed in SEAR (Surfactant Enhanced Aquifer Remediation) processes. It is given by the following empirical relation.

$$
\left(\frac{p_{b}}{p_{c}}\right)^{\omega_{1}}=\left(1-\frac{s-s_{r a}^{\sigma}}{1-s_{r a}^{\sigma}-s_{r o}^{\sigma}}\right) .
$$

In the above, $s_{r a}^{\sigma}$ and $s_{r o}^{\sigma}$ are the residual saturations for the aqueous and oleic phases respectively at interfacial tension $\sigma, \omega_{1}$ is a curve fitting parameter called the pore-size index and $p_{b}$ is called the entry pressure. The entry pressure changes with the interfacial tension of the system. This effect is captured using a Leverett function scaling procedure in which $p_{b}$ is replaced by $\sigma \omega_{2}(\phi / K)^{1 / 2}$ where $\omega_{2}$ is a proportionality constant and $K$ is the geometric mean of the horizontal permeabilities in the $x$ and $y$ directions. Thus $p_{b}$ implicitly captures the relationship between capillary pressure $p_{c}$ and surfactant concentration $\Gamma$ via the interfacial tension $\sigma=\sigma(\Gamma)$. Hence the capillary pressure is modeled as a function of porosity, permeability, the aqueous phase saturation and the surfactant concentration.

From surface chemistry we know that surfactants improve the mobilization of trapped oil by reducing the interfacial tension forces which in turn leads to the reduction of the capillary pressure between oil and water. But this also leads to a reduction of the residual saturations and hence an increase in the relative permeabilities of the two phases. In fact, for IFT values below $0.1 \mathrm{mN} / \mathrm{m}$ (milliNewton per meter) the relative permeabilities of both the phases increase substantially due to a decrease of the residual saturations of the two phases with an increase in IFT (captured through the capillary number, $N_{c}$ ). This effect on the relative permeabilities and the residual saturations can be captured through the relative permeability curves. The relative permeability curves are modeled following the approach of Amaefule and Handy [55] as

$$
\begin{aligned}
& k_{r a}^{\sigma}=\left(\frac{s-s_{r a}^{\sigma}}{1-s_{r a}^{\sigma}}\right)\left\{2.5 s_{r a}^{\sigma}\left[\left(\frac{s-s_{r a}^{\sigma}}{1-s_{r a}^{\sigma}}\right)^{2}-1\right]+1\right\}, \\
& k_{r o}^{\sigma}=\left(1-\frac{s-s_{r a}^{\sigma}}{1-s_{r a}^{\sigma}-s_{r o}^{\sigma}}\right)\left\{5 s_{r o}^{\sigma}\left[\left(1-\frac{s-s_{r a}^{\sigma}}{1-s_{r a}^{\sigma}-s_{r o}^{\sigma}}\right)-1\right]+1\right\} .
\end{aligned}
$$

Here $k_{r a}^{\sigma}$ and $k_{r o}^{\sigma}$ are the respective relative permeabilities of the aqueous and the oleic phases at an interfacial tension of $\sigma$.

To use the above models for the capillary pressure, eq. (19), and the relative permeability, eq. (20), we need to be able to determine the variation in the residual saturations of the phases with changes in the interfacial tension. For this purpose, we use the empirical expressions that relate the IFT and the residual saturations 
using the capillary number (see [55]) given as follows

$$
\begin{gathered}
s_{r o}^{\sigma}= \begin{cases}s_{r o}^{\sigma 0} & N_{c}<N_{c o} \\
s_{r o}^{\sigma 0}\left(\frac{N_{c o}}{N_{c}}\right)^{0.5213} & N_{c} \geq N_{c o}\end{cases} \\
s_{r a}^{\sigma}= \begin{cases}s_{r a}^{\sigma 0} & N_{c}<N_{c a o} \\
s_{r a}^{\sigma 0}\left(\frac{N_{c a o}}{N_{c}}\right)^{0.1534} & N_{c} \geq N_{c a o}\end{cases}
\end{gathered}
$$

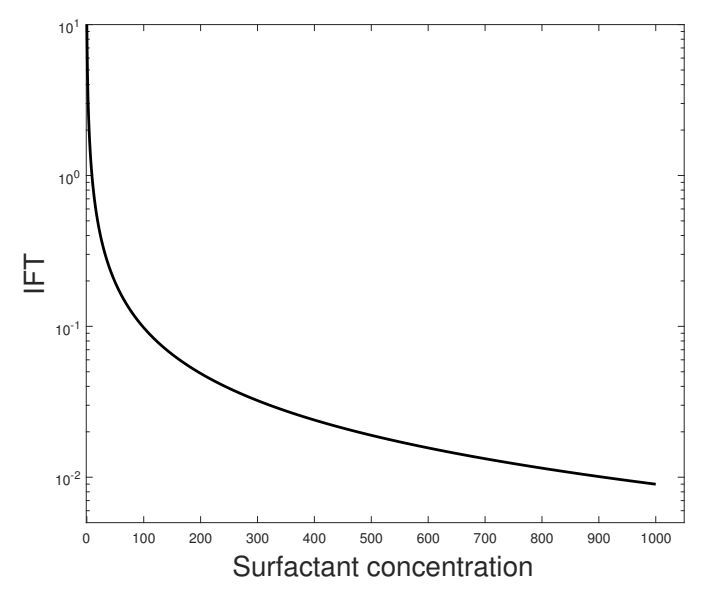

Figure 2: Interfacial tension of oil/brine as a function of surfactant concentration.

Here the exponents are obtained through curve fitting on experimental data. $N_{c}=\frac{\mu|\mathbf{v}|}{\sigma}$ is the capillary number calculated from the displacing fluid viscosity $\mu$, Darcy velocity $\mathbf{v}$ and interfacial tension, $\sigma$. The oleic and the aqueous phase capillary numbers at interfacial tension, $\sigma 0$, are $N_{c o}$ and $N_{c a o}$ respectively. Also, $s_{r a}^{\sigma 0}$ and $s_{r o}^{\sigma 0}$ are the residual saturations of the aqueous and oleic phases respectively at interfacial tension $\sigma 0$. Finally, to complete the model we use the following empirical relationship between the surfactant concentration and the interfacial tension that closely resembles the behavior observed in laboratory experiments (see $[56])$,

$$
\sigma=\frac{10.001}{\Gamma+1}-0.001
$$

The flow and transport equations given by eqs. (17a)-(17d) combined with the constitutive relations given by eqs. (18)-(22) form a complete mathematical model for the SP flooding.

A similar model for the polymer flooding is obtained by treating it as a simplified case of the above system. One of the key differences will be in the definition of the global pressure, $p^{\text {pol }}$, which is now given by

$$
\begin{aligned}
p^{\mathrm{pol}}=\frac{1}{2}\left(p_{o}+p_{a}\right) & +\frac{1}{2} \int_{s_{c}}^{s}\left(\hat{\lambda}_{o}(\zeta, c)-\hat{\lambda}_{a}(\zeta, c)\right) \frac{d p_{c}}{d \zeta}(\zeta) d \zeta \\
& -\frac{1}{2} \int\left(\int_{s_{c}}^{s} \frac{\partial}{\partial c}\left(\hat{\lambda}_{o}(\zeta, c)-\hat{\lambda}_{a}(\zeta, c)\right) \frac{d p_{c}}{d \zeta}(\zeta) d \zeta\right)\left(\frac{\partial c}{\partial x} d x+\frac{\partial c}{\partial y} d y\right)+C^{\mathrm{pol}}
\end{aligned}
$$

where $C^{\text {pol }}$ is again some reference pressure which replaces the integration constant. The corresponding constitutive relations for the relative permeabilities, $k_{r a}(s, c)$ and $k_{r o}(s)$, and the capillary pressure $p_{c}(s)$ are 
obtained from the Corey-type imbibition relations ([57] and [58]).

$$
\begin{aligned}
k_{r a} & =\left(\frac{s-s_{r a}^{\sigma 0}}{1-s_{r a}^{\sigma 0}}\right)^{3.5}, \\
k_{r o} & =\left(1-\frac{s-s_{r a}^{\sigma 0}}{1-s_{r a}^{\sigma 0}-s_{r o}^{\sigma 0}}\right)^{2}\left[1-\left(\frac{s-s_{r a}^{\sigma 0}}{1-s_{r a}^{\sigma 0}-s_{r o}^{\sigma 0}}\right)^{1.5}\right], \\
p_{c} & =p_{d}\left(\frac{s-s_{r a}^{\sigma 0}}{1-s_{r a}^{\sigma 0}}\right)^{-0.25},
\end{aligned}
$$

where $k_{r a}$ and $k_{r o}$ are the relative permeability functions of the aqueous phase and the oleic phase respectively when the interfacial tension between the two phases is given by $\sigma 0$. Also, $p_{d}$ is the displacement pressure or entry pressure, as defined before in eq. (19). The exponents used in eqs. (24a)-(24c) and the value of $p_{d}$ are usually determined by fitting curves to equilibrium experimental data.

Alternatively, the Van Genuchten model [59] and the subsequent generalization done by Parker et al. [60] are also available

$$
\begin{aligned}
& k_{r a}(s)=s_{e}^{1 / 2}\left(1-\left(1-s_{e}^{1 / m}\right)^{m}\right)^{2}, \\
& k_{r o}(s)=\left(1-s_{e}\right)^{1 / 2}\left(1-s_{e}^{1 / m}\right)^{2 m}, \\
& p_{c}(s)=\frac{1}{\alpha_{0}}\left(s_{e}^{-1 / m}-1\right)^{1-m},
\end{aligned}
$$

where $s_{e}=\left(s-s_{r a}^{\sigma 0}\right) /\left(1-s_{r a}^{\sigma 0}\right)$ is called the effective saturation. For the sake of simplicity, in eqs. (25a) $-(25 \mathrm{c})$ the residual wetting phase saturation, $s_{r a}^{\sigma 0}$ and the residual non-wetting phase saturation, $s_{r o}^{\sigma 0}$ may be taken to be zero with the parameters $m=2 / 3$ and $\alpha_{0}=0.125$ (see [61]). We have performed numerical simulations of polymer flooding with both of these models and the results have been found to be qualitatively similar.

\section{$3 \quad$ Numerical method}

The governing eqs. (17a)-(17d) form a coupled system of nonlinear partial differential equations. The total mobility $\lambda(s, c, \Gamma)$ appearing in the pressure eq. (17a) depends on the solution of the transport equations. Also, the transport eqs. (17b)-(17d) depend on the total velocity $\mathbf{v}$, thus introducing a coupling with the elliptic pressure equation. Additionally, since the phase mobilities (and so the fractional flow functions) are nonlinear functions of the water saturation and the species concentrations, the equations are nonlinear. For each time step, this coupled system of equations is solved using the following sequential procedure. First, the initial values of $s, c$ and $\Gamma$ are used as input for solving the elliptic eq. (17a) for the global pressure $p$ and the total velocity $\mathbf{v}$ using a non-traditional finite element formulation which is described in section 3.2. Next, $\mathbf{v}$ is used as an input in the transport eqs. (17b)-(17d) to advance the current values of triplet $(s, c, \Gamma)$ to the next time level by solving these equations using a combination of the MMOC and an implicit-time finite difference scheme. This sequential time-stepping process repeats until a suitable stopping criterion is reached. The numerical treatment of the nonlinearity has been discussed in section 3.3 on the finite difference numerical scheme.

Below, section 3.1 describes two different computational grids that are used for solving the system of equations. The transport equations are solved on a uniform Cartesian grid that is referred to as the FD (finite difference) grid. The pressure equation is solved using a non-traditional finite element formulation on a fixed mesh, composed of triangular elements, that is referred to as the FE (finite element) grid. Section 3.2 describes the finite element formulation in detail. The MMOC based finite difference method used for the transport equations is presented in section 3.3. This is followed by section 3.4 on numerically generating scalar absolute permeability fields to accurately model multiscale rock heterogeneity. Finally, the algorithm, the pseudocode, and the flowchart of the method are presented in section 3.5. 


\subsection{Computational grid}

The numerical simulations have been performed in two types of domains: quarter five-spot and rectilinear. The quarter five-spot domain $\Omega=[0,1]^{2}$ has an injection well at $(0,0)$, a production well at $(1,1)$ and no flow conditions on the rest of the boundary, as shown in Fig. $3(\mathrm{a})$. The rectilinear flow domain $\Omega=[0,1]^{2}$ has a line source at $x=0$ and a line sink at $x=1$. No flow conditions are specified on the remaining top and bottom horizontal boundaries, as shown in Fig. 3(b).

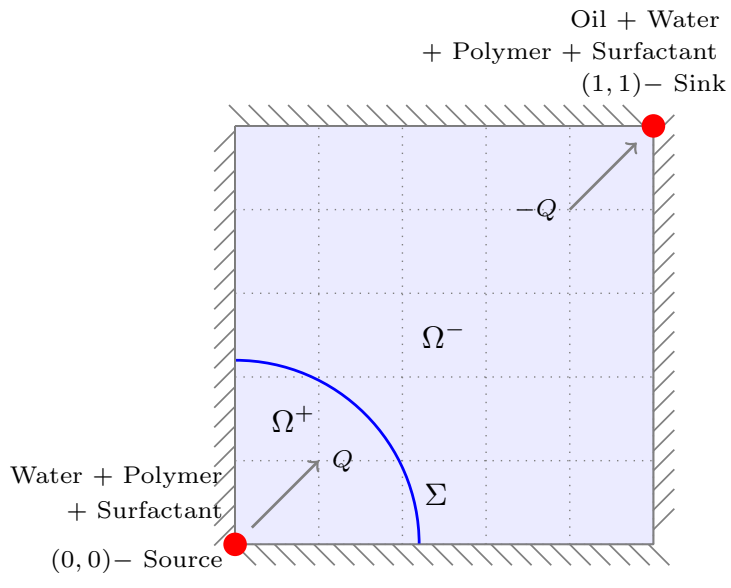

(a) Schematic of a quarter five-spot reservoir

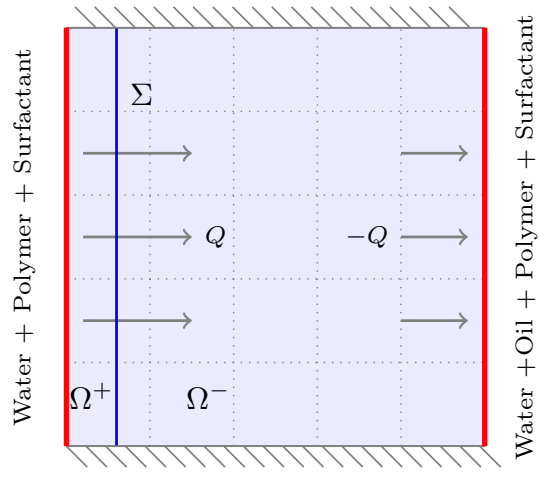

(b) Schematic of a rectilinear flow reservoir

Figure 3: Schematic of two different types of simulation domains where $Q$ is the injection/production rate

The FD grid used to solve the system of coupled transport eqs. (17b)- $(17 \mathrm{~d})$ is constructed by partitioning the domain $\Omega$ into uniform rectangular cells. Given positive integers $I, J \in \mathcal{Z}^{+}$, set $\Delta x=\left(x_{\max }-x_{\min }\right) / I=$ $1 / I$ and $\Delta y=\left(y_{\max }-y_{\min }\right) / J=1 / J$. We define a uniform Cartesian grid $\left(x_{i}, y_{j}\right)=(i \Delta x, j \Delta y)$ for $i=0, \ldots, I$ and $j=0, \ldots, J$. Each $\left(x_{i}, y_{j}\right)$ is called a grid point. For the case $i=0, I$ or $j=0, J$, a grid point is called a boundary point, otherwise it is called an interior point. The grid size is defined as $h=\max (\Delta x, \Delta y)>0$, see Fig. 4(a). In our numerical simulations we have assumed $\Delta x=\Delta y=h$.

$(1,1)-\operatorname{Sink}$

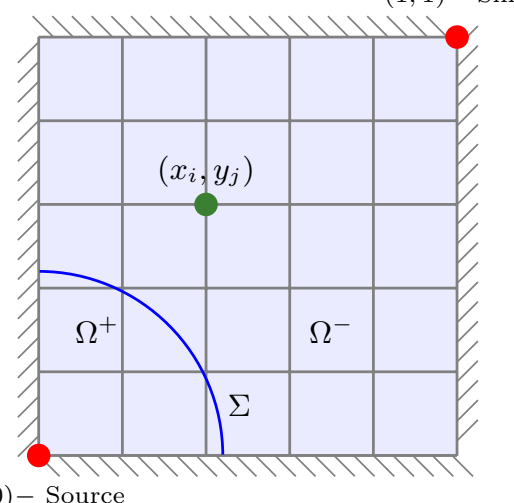

$(0,0)$ - Source

(a) Uniform FD grid for the transport equations
$(1,1)-\operatorname{Sink}$

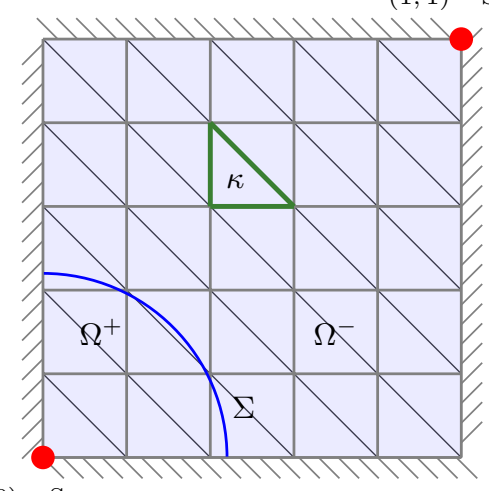

$(0,0)$ - Source

(b) Uniform FE mesh for the pressure equation

Figure 4: Discretization of the computational domain for (a) the Transport Equations and (b) the Pressure Equation. 
The FE grid used to solve the elliptic flow eq. (17a) for the global pressure, is constructed by introducing triangulations within the FD grid. This means every rectangular region $\left[x_{i}, x_{i+1}\right] \times\left[y_{j}, y_{j+1}\right]$ is cut into two pieces of right triangular regions: one is bounded by $x=x_{i}, y=y_{j}$ and $y=\frac{y_{j+1}-y_{j}}{x_{i}-x_{i+1}}\left(x-x_{i+1}\right)+y_{j}$, the other is bounded by $x=x_{i+1}, y=y_{j+1}$ and $y=\frac{y_{j+1}-y_{j}}{x_{i}-x_{i+1}}\left(x-x_{i+1}\right)+y_{j}$. Union of all those triangular regions gives a uniform triangulation, $L^{h}=\{\kappa \mid \kappa$ is a triangular element $\}$, see Fig. $4(\mathrm{~b})$. We can also choose the hypotenuse to be $y=\frac{y_{j+1}-y_{j}}{x_{i+1}-x_{i}}\left(x-x_{i}\right)+y_{j}$ and get a different uniform triangulation from the same Cartesian grid.

\subsection{Pressure equation}

The elliptic problem for the pressure is given by

$$
\begin{array}{rlrl}
-\boldsymbol{\nabla} \cdot(\mathbf{K}(\mathbf{x}) \lambda(s, c, \Gamma) \nabla p) & =\tilde{q}, & & \mathbf{x} \in \Omega \backslash \Sigma \\
(\mathbf{K}(\mathbf{x}) \lambda(s, c, \Gamma) \boldsymbol{\nabla} p) \cdot \hat{\mathbf{n}} & =0, & \mathbf{x} \in \partial \Omega
\end{array}
$$

where $\tilde{q}=q_{a}+q_{o}$ and $\Sigma$ denotes the union of the interfaces that separate $\Omega$ into several subdomains. These interfaces are a macroscopic averaged picture. Also, at any arbitrary time during the flooding process, there might be many such interfaces in the domain generated dynamically due to the flow and hence there can be several subdomains created inside $\Omega$. However, for simplicity of exposition, we have taken only two subdomains, $\Omega^{+}$and $\Omega^{-}$separated by an interface $\Omega$ which also happens to be the initial configuration of the domain for most of our injection policies in this paper as we will see later.

The above problem is solved using a non-traditional finite element formulation (see [62]) with a uniform triangulated non-body-fitted grid, which is second order accurate in the $L^{\infty}$ norm for matrix coefficient elliptic equations with discontinuities across interfaces. We prescribe the following kinematic condition at the interface $\Sigma$

$$
[\mathbf{K}(\mathbf{x}) \lambda(s, c, \Gamma) \boldsymbol{\nabla} p \cdot \hat{\mathbf{n}}]_{\Sigma}=0,
$$

where $\hat{\mathbf{n}}$ is the outward unit normal which points from $\Omega^{-}$to $\Omega^{+}$. We assume the boundary $\partial \Omega$ and the interface $\Sigma$ to be Lipschitz continuous. Hence a unit normal vector, $\hat{\mathbf{n}}$ can be defined a.e. on $\Sigma$. We generalize the weak formulation for the elliptic equation in the usual Sobolev spaces $H^{1}(\Omega)$ with $\psi \in H^{1}(\Omega)$ as

$$
\int_{\Omega^{+}} \mathbf{K} \lambda \boldsymbol{\nabla} p \boldsymbol{\nabla} \psi+\int_{\Omega^{-}} \mathbf{K} \lambda \boldsymbol{\nabla} p \boldsymbol{\nabla} \psi-\int_{\partial \Omega} \mathbf{K} \lambda \psi \boldsymbol{\nabla} p \cdot \hat{\mathbf{n}}=\int_{\Omega} \tilde{q} \psi .
$$

The rationale behind adopting this non-traditional finite element formulation is many-fold. The coefficient of the capillary dissipation term in the water saturation equation, given by $\mathbf{K} \lambda_{o} f_{a} \frac{\partial p_{c}}{\partial s}$, vanishes at extreme values of the water saturation $s=s_{r a}$ and $s=1-s_{r o}$ because $f_{a}\left(s=s_{r a}\right)=0$ and $\lambda_{o}\left(s=1-s_{r o}\right)=0$. This makes it a degenerate diffusion term and the corresponding transport equation becomes nonlinear hyperbolic in nature at the points of degeneracy. It is well known that nonlinear hyperbolic equations can spontaneously generate shocks or discontinuities in the solution. This discontinuity in $s$ gets transmitted to the coefficient $\mathbf{K} \lambda(s, c, \Gamma)$ of the elliptic global pressure equation via the total mobility term. The nontraditional finite element formulation has been adopted as it can efficiently handle such discontinuities in the elliptic coefficient by incorporating it in the basis functions and redefining the weak formulation. In the region where the coefficient has no discontinuity, the basis functions automatically reduce to the linear basis functions of a traditional finite element formulation. Also, this formulation uses a fixed finite element mesh, thus avoiding the expensive computation of generating a mesh every time step as would be necessary for a traditional finite element method that uses a body-fitted grid. The method ensures that the resulting linear system is positive definite and it achieves second order accuracy in the $L^{\infty}$ norm.

In the numerical results presented in this paper, the degeneracy in the capillary dissipation term has been avoided because of the restriction $0<s_{r a}<s<1-s_{r o}<1$. This is a realistic physical assumption which means that the initial water saturation in the reservoir (connate water saturation) is not as low 
as the residual water saturation and during the displacement process the water saturation does not get reduced to the residual water saturation in finite time. In other words, the water saturation is always strictly bounded below and above by the water residual saturation and the complement of the oil residual saturation respectively for that reservoir. However, the initial saturation profile $s(\mathbf{x}, 0)=s_{0}(\mathbf{x})$ has a discontinuity. The non-traditional formulation can treat this discontinuity efficiently and automatically switch to a more traditional finite element formulation when the dissipation introduced by the capillary pressure term smoothens out the the initial discontinuity in the water saturation. Additionally due to the nonlinearity of the system and the heterogeneous permeability, if discontinuities were to arise in the solution over time then the non-traditional finite element formulation is capable of handling them efficiently. The method is explained in detail below.

The elements, $\kappa$ of triangulation, $L^{h}$, introduced in section 3.1 are classified into regular cells and interface cells. We call $\kappa$ a regular cell if its vertices are in the same subdomain and an interface cell when its vertices belong to different subdomains. For an interface cell, $\kappa=\kappa^{+} \cup \kappa^{-}$where $\kappa^{+}$and $\kappa^{-}$are separated by a line segment $\Sigma_{k}^{h}$, obtained by joining the two points where the interface $\Sigma$ intersects the sides or the vertices of that interface cell. A set of grid functions, $H^{1, h}=\left\{\omega^{h} \mid \omega^{h}=\omega_{i, j} ; 0 \leq i \leq I, 0 \leq j \leq J\right\}$ are defined on the grid points of the mesh $L^{h}$. An extension operator $U^{h}: H^{1, h} \rightarrow H^{1}(\kappa)$ is constructed as follows. For any $\phi^{h} \in H^{1, h}, U^{h}\left(\phi^{h}\right)$ is a piecewise linear function and matches $\phi^{h}$ on the grid points. In a regular cell, it is a linear function that interpolates the values of $\phi^{h}$ at the grid points. In an interface cell, it consists of two pieces of linear functions, one each defined on $\kappa^{+}$and $\kappa^{-}$. The location of the discontinuity of the extended function $U^{h}\left(\phi^{h}\right)$ in an interface cell is on the line segment $\Sigma_{k}^{h}$. Hence an interface jump condition on the pressure, $p$, if there is one, can be imposed on the two end points of this line segment at $\{\partial \kappa\} \cap\left\{\Sigma_{k}^{h}\right\}$ while the interface jump condition, eq. (27), is imposed at the middle point of $\Sigma_{k}^{h}$. The construction of such extension operators and proof of their uniqueness can be found in the literature $[63,64,62]$.

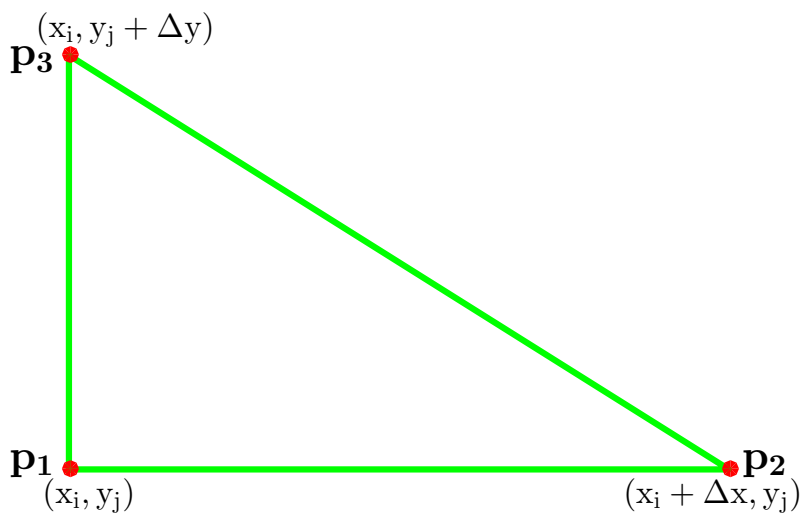

Figure 5: Regular cell

For every $\phi^{h} \in H^{1, h}$, we can define a unique extension $U^{h}\left(\phi^{h}\right)$ from knowing the position of the interface $\Sigma$. For a regular cell, as in Fig. 5 we construct $U^{h}\left(\phi^{h}\right)$ as

$$
U^{h}\left(\phi^{h}\right)=\phi\left(p_{1}\right)+\frac{\phi\left(p_{2}\right)-\phi\left(p_{1}\right)}{\Delta x}\left(x-x_{i}\right)+\frac{\phi\left(p_{3}\right)-\phi\left(p_{1}\right)}{\Delta y}\left(y-y_{j}\right)
$$

For an interface cell, $\kappa$ there are two cases. Case 1 is when the interface cuts through two legs of $\kappa$ (as in Fig. 6), and Case 2 is when the interface cuts through one leg and the hypotenuse of $\kappa$ (as in Fig. 7). As an example, we construct the extension function for Case 1 and the construction for Case 2 follows similarly. Then for Case 1,

$$
U^{h}\left(\phi^{h}\right)=\left\{\begin{array}{l}
\phi\left(p_{1}\right)+\phi_{x}^{+}\left(x-x_{i}\right)+\phi_{y}^{+}\left(y-y_{j}\right) \quad \text { for }(x, y) \in \kappa^{+}, \\
\phi\left(p_{2}\right)+\phi_{x}^{-}\left(x-x_{i}-\Delta x\right)+\phi_{y}^{-}\left(y-y_{j}\right) \quad \text { for }(x, y) \in \kappa^{-}
\end{array}\right.
$$




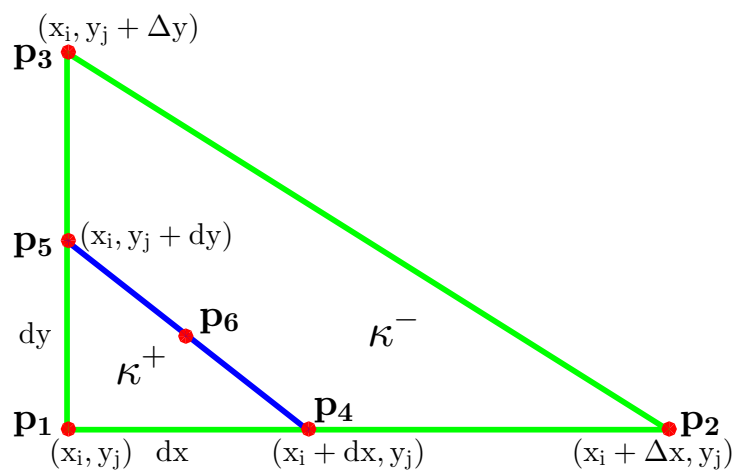

(a) Formulation for $\kappa^{+}$

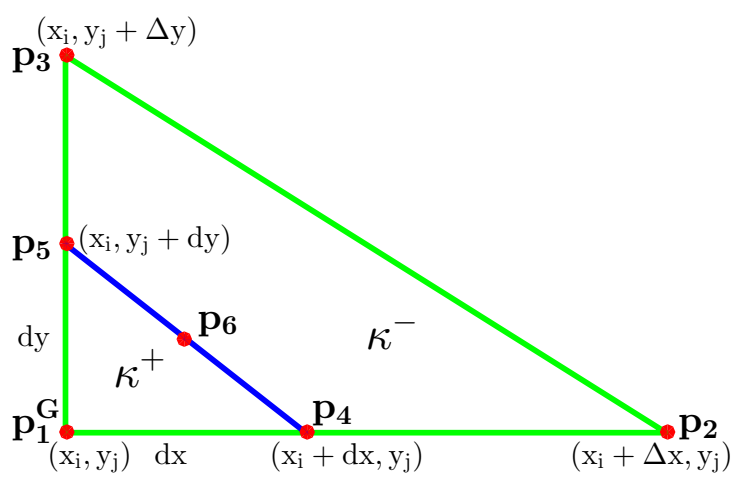

(b) Ghost point formulation for $\kappa^{-}$

Figure 6: Case 1 - the interface cutting through two legs

where for $(x, y) \in \kappa^{+}$(as in Fig. 6(a)),

$$
\phi_{x}^{+}=\frac{\phi\left(p_{4}\right)-\phi\left(p_{1}\right)}{d x} \quad \& \quad \phi_{y}^{+}=\frac{\phi\left(p_{5}\right)-\phi\left(p_{1}\right)}{d y},
$$

and for $(x, y) \in \kappa^{-}$(as in Fig. 6(b)),

$$
\phi_{x}^{-}=\frac{\phi\left(p_{2}\right)-\phi\left(p_{4}\right)}{\Delta x-d x} \quad \& \quad \phi_{y}^{-}=\frac{\phi\left(p_{3}\right)-\phi\left(p_{5}\right)}{\Delta y-d y} .
$$

Above it is assumed that the extensions of $p_{3} p_{5}$ and $p_{2} p_{4}$ intersect at a ghost point, $p_{1}^{G}$ and hence

$$
\frac{\phi\left(p_{1}^{G}\right)-\phi\left(p_{4}\right)}{d x}=\frac{\phi\left(p_{4}\right)-\phi\left(p_{2}\right)}{\Delta x-d x} \quad \& \quad \frac{\phi\left(p_{1}^{G}\right)-\phi\left(p_{5}\right)}{d y}=\frac{\phi\left(p_{1}^{G}\right)-\phi\left(p_{3}\right)}{\Delta y} .
$$

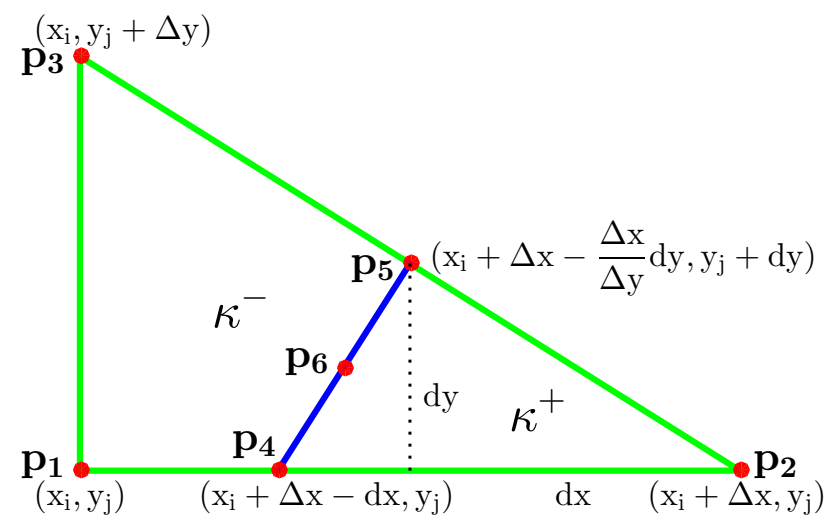

Figure 7: Case 2 - the interface cutting through one leg and the hypotenuse

We note that $\phi_{x}^{+}$and $\phi_{x}^{-}$are linear functions of $\phi\left(p_{1}\right), \phi\left(p_{2}\right), \phi\left(p_{3}\right)$ and $\phi\left(p_{4}\right)$ from eq. (31) and eq. (32). We rewrite eq. (33) as

$$
\begin{array}{r}
\phi\left(p_{5}\right)=\phi\left(p_{1}^{G}\right)-\frac{d y}{\Delta y}\left(\phi\left(p_{1}^{G}\right)-\phi\left(p_{3}\right)\right), \\
\phi\left(p_{1}^{G}\right)=\phi\left(p_{4}\right)+\frac{d x}{\Delta x-d x}\left(\phi\left(p_{4}\right)-\phi\left(p_{2}\right)\right) .
\end{array}
$$


Using eq. (34) and eq. (35) in eq. (31) and eq. (32), $\phi_{y}^{+}$and $\phi_{y}^{-}$respectively can be obtained as linear functions of $\phi\left(p_{1}\right), \phi\left(p_{2}\right), \phi\left(p_{3}\right)$ and $\phi\left(p_{4}\right)$. The kinematic condition, eq. (27), is imposed as given below,

$$
0=\mathbf{K}^{+} \lambda^{+} \boldsymbol{\nabla} \phi^{+} \cdot \hat{\mathbf{n}}-\mathbf{K}^{-} \lambda^{-} \boldsymbol{\nabla} \phi^{-} \cdot \hat{\mathbf{n}} .
$$

Given $\hat{\mathbf{n}}=\left(n_{1}, n_{2}\right)=\left(\frac{-d y}{\sqrt{d x^{2}+d y^{2}}}, \frac{-d x}{\sqrt{d x^{2}+d y^{2}}}\right)$ and the permeability $\mathbf{K}=\left(\begin{array}{cc}k & 0 \\ 0 & k\end{array}\right)$ for an isotropic porous medium, eq. (36) reduces to

$$
k^{+} \lambda^{+} \phi_{x}^{+} n_{1}+k^{+} \lambda^{+} \phi_{y}^{+} n_{2}=k^{-} \lambda^{-} \phi_{x}^{-} n_{1}+k^{-} \lambda^{-} \phi_{y}^{-} n_{2} .
$$

Using eq. (37), $\phi\left(p_{4}\right)$ is eliminated so that, $\phi_{x}^{+}, \phi_{y}^{+}, \phi_{x}^{-}$and $\phi_{y}^{-}$are obtained uniquely as linear combinations of $\phi\left(p_{1}\right), \phi\left(p_{2}\right)$ and $\phi\left(p_{3}\right)$ in such a way that the coefficients are finite and independent of $\phi^{h}$. Thus the extension operator $U^{h}$, given by eq. (30), for Case 1 of an interface cell becomes well defined as in the case of a regular cell given by eq. (29). Similarly, $U^{h}\left(\phi^{h}\right)$ can be defined for Case 2 of an interface cell (Fig. 7) with finite and well defined coefficients in the following way,

$$
U^{h}\left(\phi^{h}\right)=\left\{\begin{array}{lr}
\phi\left(p_{2}\right)+\phi_{x}^{+}\left(x-x_{i}-\Delta x\right)+\phi_{y}^{+}\left(y-y_{j}\right) & \text { for }(x, y) \in \kappa^{+}, \\
\phi\left(p_{1}\right)+\phi_{x}^{-}\left(x-x_{i}\right)+\frac{\phi\left(p_{3}\right)-\phi\left(p_{1}\right)}{\Delta y}\left(y-y_{j}\right) & \text { for }(x, y) \in \kappa^{-},
\end{array}\right.
$$

where

$$
\begin{aligned}
& \phi_{x}^{+}=\frac{\phi\left(p_{2}\right)-\phi\left(p_{4}\right)}{d x} \& \phi_{y}^{+}=\frac{\phi\left(p_{5}\right)-\phi\left(p_{2}\right)}{d y}+\frac{\phi\left(p_{2}\right)-\phi\left(p_{4}\right)}{d x} \frac{\Delta x}{\Delta y}, \\
& \phi_{x}^{-}=\frac{\phi\left(p_{4}\right)-\phi\left(p_{1}\right)}{\Delta x-d x} .
\end{aligned}
$$

Using the extension functions as defined above, the weak formulation eq. (28) reduces to finding a discrete function $\phi^{h} \in H^{1, h}$ such that it satisfies eq. (26b) on the boundary points and so that for all $\psi^{h} \in H^{1, h}$,

$$
\begin{array}{r}
\sum_{K \in L^{h}}\left(\int_{K^{+}} \mathbf{K} \lambda \nabla U^{h}\left(\phi^{h}\right) \nabla U^{h}\left(\psi^{h}\right)+\int_{K^{-}} \mathbf{K} \lambda \boldsymbol{\nabla} U^{h}\left(\phi^{h}\right) \boldsymbol{\nabla} U^{h}\left(\psi^{h}\right)-\int_{\partial K} \mathbf{K} \lambda U^{h}\left(\psi^{h}\right) \boldsymbol{\nabla} U^{h}\left(\phi^{h}\right) \cdot \hat{\mathbf{n}}\right) \\
=\sum_{K \in L^{h}}\left(\int_{K^{+}} \tilde{q} U^{h}\left(\psi^{h}\right)+\int_{K^{-}} \tilde{q} U^{h}\left(\psi^{h}\right)\right) .
\end{array}
$$

It can be shown that if $\mathbf{K}(\mathbf{x})$ is positive definite, then the matrix obtained for the linear system of the discretized weak form, eq. (41), is also positive definite and is therefore invertible.

\subsection{Transport equations}

As mentioned earlier, the transport eqs. (17b)-(17d), are solved using the MMOC. In convection dominated diffusion problems like these, for any reasonable fluid injection rate and capillary dissipation strength, the flow is essentially along the characteristics. The principal gain from using the MMOC procedure to solve these problems is in the time truncation, as the solution changes much less rapidly in the characteristic $\tau$ direction than in the $t$ direction. Larger time steps can be used in these methods, thus improving the efficiency without adversely affecting the accuracy. Additionally there is no stability limitation on the size of $\Delta t$ if an implicit time marching scheme is adopted as implicit matrix solvers are usually much less sensitive to numerical instability. Next, we present the procedure of MMOC. 
The transport term, $\phi s_{t}+\frac{\partial f_{a}}{\partial s} \mathbf{v} \cdot \boldsymbol{\nabla} s$ in eq. (17b) is replaced by a derivative along its characteristic direction in the following way

$$
\frac{\partial}{\partial \tau_{s}}=\frac{1}{\psi_{s}}\left(\phi \frac{\partial}{\partial t}+\frac{\partial f_{a}}{\partial s} \mathbf{v} \cdot \nabla\right),
$$

where $\tau_{s}$ is used to parametrize the characteristics. Here $\psi_{s}$ is a suitable normalization that simplifies the numerical discretization of the characteristic derivative and is defined by

$$
\psi_{s}=\left[\phi^{2}+\left(\frac{\partial f_{a}}{\partial s}\right)^{2}|\mathbf{v}|^{2}\right]^{1 / 2} .
$$

Then eq. (17b) is equivalently written in the form

$$
\psi_{s} \frac{\partial s}{\partial \tau_{s}}+\nabla \cdot\left(D \frac{\partial p_{c}}{\partial s} \boldsymbol{\nabla} s\right)=\tilde{g_{s}}-\frac{\partial f_{a}}{\partial c} \mathbf{v} \cdot \boldsymbol{\nabla} c-\frac{\partial f_{a}}{\partial \Gamma} \mathbf{v} \cdot \nabla \Gamma-\nabla \cdot\left(D \frac{\partial p_{c}}{\partial \Gamma} \nabla \Gamma\right),
$$

where $\tilde{g_{s}}=\left(1-f_{a}\right) Q \delta\left(\mathbf{x}-\mathbf{x}^{i}\right)$ is the point source term.

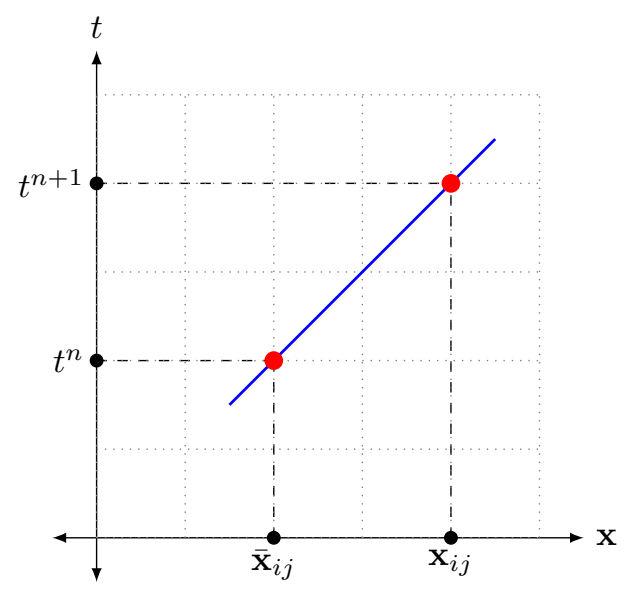

Figure 8: Discrete approximation of the characteristic curve from $\overline{\mathbf{x}}_{i j}$ to $\mathbf{x}_{i j}$ in $1 \mathrm{D}$

For numerical computation, we use the spatial grid described in section 3.1 and the time interval $[0, T]$ is uniformly divided into $N$ subintervals of length $\Delta t$, such that $t^{n}=n \Delta t$ and $T=N \Delta t$. Then we denote the grid values of the variables by $w_{i j}^{n}=w\left(\mathbf{x}_{i j}, t^{n}\right)$ where $\mathbf{x}_{i j}=\mathbf{x}(i h, j h)$. Consider that the solution is known at some time $t^{n}$ and the solution at a subsequent time $t^{n+1}$ needs to be computed. Then starting from any point $\left(\mathbf{x}_{i j}, t^{n+1}\right)$ we trace backward along the characteristics to a point $\left(\overline{\mathbf{x}}_{i j}, t^{n}\right)$ where the solution is already known.

Let the characteristics which originate from the point $\overline{\mathbf{x}}_{i j}$ at time $t^{n}$ reach the point $\mathbf{x}_{i j}$ at time $t^{n+1}$ (see Fig. 8). From the equation of the characteristic curves given by

$$
\frac{d \mathbf{x}}{d \tau_{s}}=\frac{1}{\phi} \frac{\partial f_{a}}{\partial s} \mathbf{v}
$$

we can use numerical discretization to obtain an approximate value of $\overline{\mathbf{x}}_{i j}$ in the following way

$$
\overline{\mathbf{x}}_{i j}=\mathbf{x}_{i j}-\frac{\partial f_{a}}{\partial s}\left(s_{i j}^{n}, c_{i j}^{n}, \Gamma_{i j}^{n}\right) \mathbf{v}_{i j}^{n} \Delta t / \phi .
$$


Using the above equation, the derivative in the characteristic direction, defined by eq. (42), is approximated by

$$
\psi_{s} \frac{\partial s}{\partial \tau_{s}} \approx \psi_{s} \frac{s\left(\mathbf{x}_{i j}, t^{n+1}\right)-s\left(\overline{\mathbf{x}}_{i j}, t^{n}\right)}{\left[\left|\mathbf{x}_{i j}-\overline{\mathbf{x}}_{i j}\right|^{2}+(\Delta t)^{2}\right]^{1 / 2}}=\phi \frac{s_{i j}^{n+1}-\bar{s}_{i j}^{n}}{\Delta t},
$$

where $s\left(\overline{\mathbf{x}}, t^{n}\right)$ is evaluated via bilinear interpolation of the approximate solution values at the nearby grid points, at time level $t^{n}=n \Delta t$. Note that in eq. (45), $\frac{\partial f_{a}}{\partial s}(s, c, \Gamma) \mathbf{v}$ is evaluated approximately as $\left(\frac{\partial f_{a}}{\partial s} \mathbf{v}\right)^{n}$ which is essentially a linearization step that freezes the nonlinear coefficient at the previous time step. In order to find a more accurate approximation of the characteristic curve passing through $\left(\mathbf{x}_{i j}, t^{n+1}\right)$, the following predictor-corrector formulation can also be adopted.

$$
\begin{aligned}
\overline{\mathbf{x}}_{i j} & =\mathbf{x}_{i j}-\frac{\partial f_{a}}{\partial s}\left(s\left(\mathbf{x}_{i j}-\frac{\partial f_{a}}{\partial s}\left(s_{i j}^{n}, c_{i j}^{n}, \Gamma_{i j}^{n}\right) \tilde{\mathbf{v}}_{i j}^{n} \Delta t / \phi\right), c_{i j}^{n}, \Gamma_{i j}^{n}\right) \tilde{\mathbf{v}}_{i j}^{n} \Delta t / \phi, \\
\tilde{\mathbf{v}}_{i j}^{n} & =2 \mathbf{v}_{i j}^{n}-\mathbf{v}_{i j}^{n-1} .
\end{aligned}
$$

It has been shown that no essential improvement in accuracy can be expected over this alternative evaluation of $s\left(\overline{\mathbf{x}}, t^{n}\right)$ even if the position $\overline{\mathbf{x}}_{i j}$ and the function $s\left(\overline{\mathbf{x}}_{i j}, t^{n}\right)$ are iterated to convergence (see [65]). In our simulations, both the formulations given by eq. (45) and eq. (47) have been used interchangeably depending on whether accuracy or speed of computation is desirable. Equation (46) leads to the following implicit-time finite difference formulation for eq. (44)

$\phi \frac{s_{i j}^{n+1}-\bar{s}_{i j}^{n}}{\Delta t}+\nabla_{h}\left(\bar{D} \nabla_{h} s\right)_{i j}^{n+1}=\left(\tilde{g}_{s}\right)_{i j}^{n}-\left(\frac{\partial f_{a}}{\partial c}\right)_{i j}^{n}\left(\mathbf{v}_{i j}^{n} \cdot \nabla_{h} c_{i j}^{n}\right)-\left(\frac{\partial f_{a}}{\partial \Gamma}\right)_{i j}^{n}\left(\mathbf{v}_{i j}^{n} \cdot \nabla_{h} \Gamma_{i j}^{n}\right)-\nabla_{h}\left(\bar{D}_{i j}^{n} \nabla_{h} \Gamma_{i j}^{n}\right)$,

where

$$
\begin{gathered}
\bar{s}_{i j}^{n}=s\left(\overline{\mathbf{x}}_{i j}, t^{n}\right) \quad \& \quad \bar{D}_{i j}^{n}=D\left(\bar{s}_{i j}^{n}, c_{i j}^{n}\right), \\
\nabla_{h}\left(\bar{D} \nabla_{h} s\right)_{i j}^{n+1}=\bar{D}_{i+1 / 2, j} \frac{s_{i+1, j}^{n+1}-s_{i, j}^{n+1}}{\Delta x^{2}}-\bar{D}_{i-1 / 2, j} \frac{s_{i, j}^{n+1}-s_{i-1, j}^{n+1}}{\Delta x^{2}} \\
+\bar{D}_{i, j+1 / 2} \frac{s_{i, j+1}^{n+1}-s_{i, j}^{n+1}}{\Delta y^{2}}-\bar{D}_{i, j-1 / 2} \frac{s_{i, j}^{n+1}-s_{i, j-1}^{n+1}}{\Delta y^{2}}, \\
\bar{D}_{i \pm 1 / 2, j}=\frac{D\left(\bar{s}_{i \pm 1, j}^{n}, c_{i \pm 1, j}^{n}\right)+D\left(\bar{s}_{i, j}^{n}, c_{i, j}^{n}\right)}{2} \& \quad \bar{D}_{i, j \pm 1 / 2}=\frac{D\left(\bar{s}_{i, j \pm 1}^{n}, c_{i, j \pm 1}^{n}\right)+D\left(\bar{s}_{i, j}^{n}, c_{i, j}^{n}\right)}{2} .
\end{gathered}
$$

In eqs. (49) and (50), the nonlinearity in the coefficient of the capillary term is approximated in a quasilinear manner by evaluating the coefficient at the previous time step. This is a standard approximation procedure that is usually adopted in many Eulerian-Lagrangian methods (see [66]).

A similar procedure is followed for discretizing the transport equations for polymer and surfactant, given by eqs. (17c) and (17d). Let us define the following

$$
\mathbf{b}=\frac{f}{s} \mathbf{v}+\frac{D}{s} \frac{\partial p_{c}}{\partial s} \nabla s+\frac{D}{s} \frac{\partial p_{c}}{\partial \Gamma} \nabla \Gamma, \quad \psi=\left(\phi^{2}+|\mathbf{b}|^{2}\right)^{1 / 2} .
$$

Also let the characteristic parameters, $\tau_{c}$ and $\tau_{\Gamma}$ respectively, be associated with the advection terms $\phi c_{t}+$ $\mathbf{b} \cdot \nabla c$ and $\phi \Gamma_{t}+\mathbf{b} \cdot \nabla \Gamma$. Even though we use different notations, the characteristic derivatives for both the parameters are defined by,

$$
\frac{\partial}{\partial \tau_{c}}=\frac{\partial}{\partial \tau_{\Gamma}}=\frac{\phi}{\psi} \frac{\partial}{\partial t}+\frac{1}{\psi} \mathbf{b} \cdot \nabla .
$$


Then using eqs. (52) and (53), the polymer and surfactant transport eqs. (17c) and (17d) are rewritten as

$$
\psi \frac{\partial c}{\partial \tau_{c}}+c \tilde{g}=\tilde{g_{c}}, \quad \psi \frac{\partial \Gamma}{\partial \tau_{\Gamma}}+\Gamma \tilde{g}=\tilde{g_{\Gamma}},
$$

where $\tilde{g_{c}}=\frac{c^{i} Q}{s} \delta\left(\mathbf{x}-\mathbf{x}^{i}\right), \tilde{g_{\Gamma}}=\frac{\Gamma^{i} Q}{s} \delta\left(\mathbf{x}-\mathbf{x}^{i}\right)$ and $\tilde{g}=\frac{Q}{s} \delta\left(\mathbf{x}-\mathbf{x}^{i}\right)$ are the source terms.

All of these lead to the following system of implicit-time finite difference formulations for the transport eqs. (17b)-(17d)

$$
\begin{aligned}
\phi \frac{s_{i j}^{n+1}-\bar{s}_{i j}^{n}}{\Delta t}+\nabla_{h}\left(\bar{D}_{i j}^{n} \nabla_{h} s_{i j}^{n+1}\right) & =\left(\tilde{g_{s}}\right)_{i j}^{n}-\left(\frac{\partial f_{a}}{\partial c}\right)_{i j}^{n}\left(\mathbf{v}_{i j}^{n} \cdot \nabla_{h} c_{i j}^{n}\right) \\
& -\left(\frac{\partial f_{a}}{\partial \Gamma}\right)_{i j}^{n}\left(\mathbf{v}_{i j}^{n} \cdot \nabla_{h} \Gamma_{i j}^{n}\right)-\nabla_{h}\left(\bar{D}_{i j}^{n} \nabla_{h} \Gamma_{i j}^{n}\right), \\
\phi \frac{c_{i j}^{n+1}-\bar{c}_{i j}^{n}}{\Delta t}+(\tilde{g})_{i j}^{n} c_{i j}^{n+1} & =\left(\tilde{g_{c}}\right)_{i j}^{n}, \\
\phi \frac{\Gamma_{i j}^{n+1}-\bar{\Gamma}_{i j}^{n}}{\Delta t}+(\tilde{g})_{i j}^{n} \Gamma_{i j}^{n+1} & =\left(\tilde{g_{\Gamma}}\right)_{i j}^{n} .
\end{aligned}
$$

\subsection{Heterogeneity}

Numerical simulations have been performed (see section 4) using several types of permeability fields to study the effect of heterogeneity and also to test the capability of the numerical method to produce accurate solutions. These include homogeneous fields, piecewise discontinuous fields and also sections of the well known SPE10 permeability field data which model the Upper Ness type and the Tarbert type rock formations. In addition to that, we also use a scalar log-normal absolute permeability field, $\mathbf{K}(\mathbf{x})$ as a model for multiscale rock heterogeneity. First we generate a Gaussian random field, $\xi(\mathbf{x})$ which we assume to be stationary (translational invariance of covariance function), isotropic (rotational invariance of covariance function) and fractal (self-similar). Hence, the distribution is characterized by a zero mean and a covariance function of the form

$$
\operatorname{cov}\left(\xi\left(\mathbf{x}_{1}\right), \xi\left(\mathbf{x}_{\mathbf{2}}\right)\right)=\chi\left|\mathbf{x}_{1}-\mathbf{x}_{\mathbf{2}}\right|^{-\beta}, 0<\beta<\infty,
$$

where $\chi$ is an appropriate proportionality constant and the scaling exponent $\beta$, called the Hurst coefficient, controls the nature of heterogeneity. With an increase in $\beta$ the covariance function decays faster and hence heterogeneities in the shorter length scales become pronounced. The LABTRAN-GEO methods, [67] are computationally efficient methods to generate Gaussian fields with a covariance function given by the power law, eq. (58). From this multiscale Gaussian field, we construct an absolute permeability field and a porosity field as

$$
\begin{aligned}
\mathbf{K}(\mathbf{x}) & =k_{0} \exp (\varpi \xi(\mathbf{x})), \\
\phi(\mathbf{x}) & =\phi_{0}+\bar{\varphi} \xi(\mathbf{x}), \bar{\varphi}>0,
\end{aligned}
$$

where $k_{0}$ is a chosen threshold, $0<\varpi \leq 1$ is a chosen scaling exponent, $\phi_{0}$ is the cutoff value and $\bar{\varphi}$ is a normalizing factor that is chosen to ensure that the porosity lies in the following range, $0.05 \leq \phi(\mathbf{x}) \leq 0.35$. The mean $\langle\mathbf{K}\rangle$ and variance $\sigma_{k}^{2}$ of the log-normal field $\mathbf{K}$ are set by the coefficients $k_{0}$ and $\varpi$. With change in $\varpi$ we get different values for the Coefficient of Variation $C V$ for permeability, $\left(C V_{k}\right)$, and porosity, $\left(C V_{\phi}\right)$. For the permeability field $\mathbf{K}$, this is defined by $C V_{k}=\sigma_{k} /\langle\mathbf{K}\rangle$. This dimensionless number $C V_{k}$ can be thought of as a measure of the heterogeneity of the field. Similar formulations have been adopted in $[68,69,70]$.

In Fig. 9(a) we present an example of such a stationary, isotropic and multiscale Gaussian field calculated with zero mean, $\beta=0.5$ and $\chi=1 /\left(1-2^{-\beta}\right)$. In Fig. $9(\mathrm{~b})$ we present a lognormal permeability field generated from this Gaussian field with $k_{0}=3.4253$ and $\varpi=1$. In our studies here, uniform porosity has been used. 


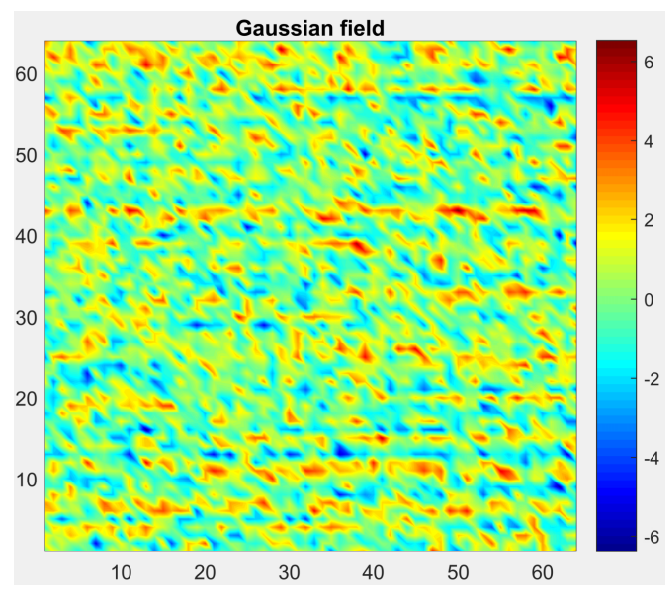

(a) Gaussian field

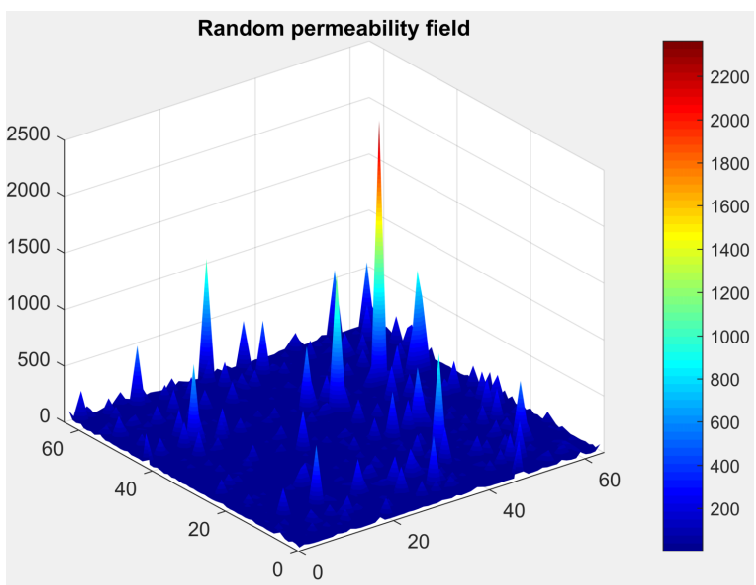

(b) Log-normal permeability

Figure 9: Stationary, isotropic, fractal Gaussian field, $\xi(\mathbf{x})$ [shown in (a)] used for generating a log-normal permeability, $\mathbf{K}(\mathbf{x})$ [shown in (b)] using eq. (59). Both fields are of size $64 \times 64$. Higher values are plotted in red and lower values in blue.

\subsection{Algorithm}

Here we outline the algorithm for the SP flood simulation. The algorithm for the polymer flood is essentially a special case of the same. The step-by-step algorithm is given as follows:

1. Define the Cartesian grid in the domain using equal, uniform grid sizes in both the axes. Generate the finite element mesh.

2. Generate a heterogeneity field on this grid.

3. Choose an initial interface separating the injected fluid from the resident fluid.

4. Set the model parameters: $\mu_{o}, \mu_{w}, s_{r o}^{\sigma 0}, s_{r a}^{\sigma 0}$.

5. Initialize the state variables $s, c$ and $\Gamma$ as

$$
s(\mathbf{x}, 0)=\left\{\begin{array}{ll}
1-s_{r o}^{\sigma 0} & x \in \Omega^{+} \\
s_{0}^{\sigma 0} & x \in \Omega^{-}
\end{array}, \quad c(\mathbf{x}, 0)=\left\{\begin{array}{ll}
c_{0} & x \in \Omega^{+} \\
0 & x \in \Omega^{-}
\end{array}, \quad \Gamma(\mathbf{x}, 0)=\left\{\begin{array}{ll}
\Gamma_{0} & x \in \Omega^{+} \\
0 & x \in \Omega^{-}
\end{array} .\right.\right.\right.
$$

6. Calculate $\sigma\left(s^{n}, \Gamma^{n}\right), \mu_{a}\left(c^{n}\right), s_{r a}\left(s^{n}, \Gamma^{n}\right), s_{r o}\left(s^{n}, \Gamma^{n}\right), \lambda_{a}\left(s^{n}, c^{n}, \Gamma^{n}\right), \lambda_{o}\left(s^{n}, \Gamma^{n}\right), \lambda\left(s^{n}, c^{n}, \Gamma^{n}\right)$ using $s^{n}$, $c^{n}, \Gamma^{n}$ which are values of $s, c$ and $\Gamma$ respectively at the $n^{\text {th }}$ time level.

7. Solve the global pressure equation to get $p^{n}$ and subsequently compute $\mathbf{v}^{n}$.

8. Use $\mathbf{v}^{n}, s^{n}, c^{n}, \Gamma^{n}$ and the quantities calculated in Step 6 , to solve for $s^{n+1}, c^{n+1}$ and $\Gamma^{n+1}$, thus completing a full time step.

9. If breakthrough is achieved: then stop; else update $n=n+1$ and repeat from Step 6 .

In order to speed up computation, sometimes a few iterations of Steps 6 and 8 are done before updating the pressure in Step 7. The pseudocode (see Algorithm 1) and flow-chart (see Fig. 10) for the procedure are given here. 

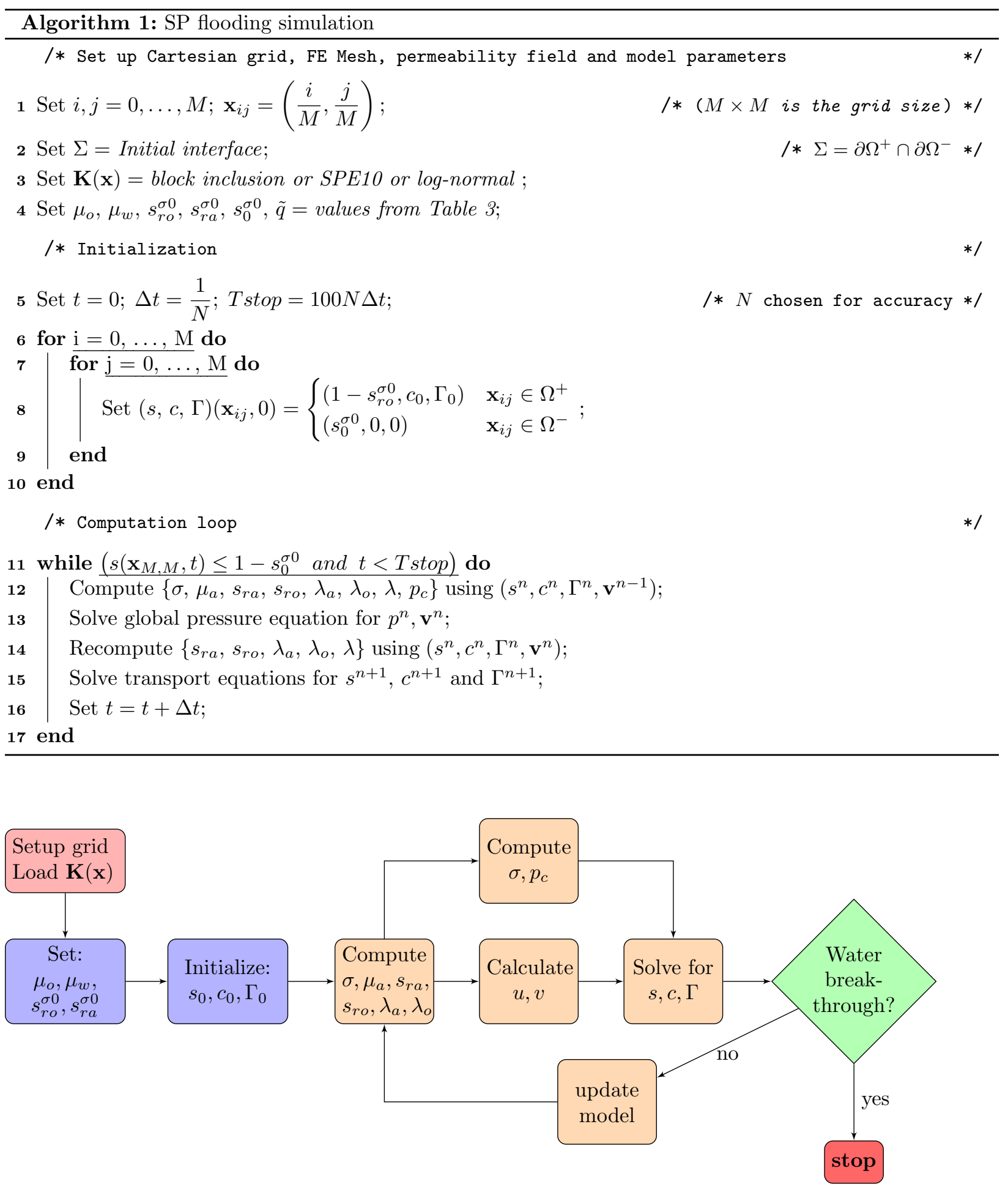

Figure 10: Flow-chart for SP flooding simulation 


\section{Results and discussion}

In section 4.1 we validate the method as well as illustrate the efficiency and accuracy of the method against an exact solution. In section 4.2 , waterflooding simulation results are presented to qualitatively validate the method against some known results in the literature and also with physically expected flow behavior. For all these waterflooding simulations the viscosity ratio, $M_{\mu}=\mu_{o} / \mu_{w}$ is kept fixed at $M_{\mu}=10$. In section 4.3 and section 4.4, we present simulation studies of polymer flood and surfactant-polymer flood respectively. These simulations are designed to explore and identify the favorable aspects of various complex chemical enhanced flooding strategies and have been carried out by varying either the viscosity ratio, $M_{\mu}$ or the mobility ratio, $M_{\lambda}=\lambda_{a}\left(s_{r o}^{\sigma}, c_{0}, \Gamma_{0}\right) / \lambda_{o}\left(s_{0}^{\sigma 0}, \Gamma_{0}\right)$ of the flooding schemes. This distinction between the viscosity ratio and the mobility ratio has been made here because the polymer in the aqueous phase exclusively affects the viscosity of the displacing phase while the surfactant in SP flood also affects the relative permeabilities. Hence in the presence of surfactant, it makes sense to compare the effective change in the mobility of the phases, while polymer floods can be studied by simply comparing the change in viscosities. The simulations in sections 4.2-4.4 have been performed on a variety of homogeneous and heterogeneous spatial permeability field data that have been either generated numerically or imported from the SPE10 benchmark problem and all other input parameters are listed in Table 3. We use two different sets of values for injection rates $Q$ and time step sizes $\Delta t$ while running simulations in a quarter five-spot geometry and in a rectilinear geometry. As reported in Table $3, Q=200$ and $\Delta t=1 / 50$ for a quarter five-spot geometry and $Q=1 / 50$ and $\Delta t=1 / 100$ for a rectilinear geometry.

The simulations have been carried out in MATLAB 2015b on a desktop computer with an Intel(R) Core(TM)2 Quad (Q9650) CPU with a clock speed of 3.00GHz, 4 GB of RAM, a GeForce GT 430/PCIe/SSE2 graphics card and running Linux Mint 13 Maya. A typical quarter five-spot flooding simulation on a $30 \times 30$ heterogeneous permeability field (SPE10) has a runtime of about 48 minutes until water breakthrough.

\subsection{Exact solution}

We consider the waterflooding problem in $\Omega=[0,1]^{2}$ and we impose the following analytical solutions

$$
\begin{aligned}
& p(x, y, t)=\left(C+\frac{x^{3}+y^{3}}{3}-\frac{x^{2}+y^{2}}{2}\right) e^{\pi t / 2} \\
& s(x, y, t)=0.3+0.7 \sin ^{2}\left(\frac{\pi t}{2}\right)\left(\frac{\sin ^{2}(2 \pi x)+\cos ^{2}(2 \pi y)}{2}\right),
\end{aligned}
$$

with $\mathbf{K}(\mathbf{x})=I, \phi(\mathbf{x})=1, \mu_{w}=\mu_{o}=1$ and $C$ is an arbitrary constant. We take $p_{c}(s)=\frac{1}{s-0.2}, k_{r w}=s_{e}^{2}$, and $k_{r o}=s_{e}\left(1-s_{e}\right)$ where $s_{e}$ is the normalized saturation defined by $s_{e}=\frac{s-0.2}{1-0.4}$. Using the solutions for pressure and saturation given by eq. (61), the source terms and the initial data are calculated for the system which in turn are used to compute the numerical solution. The numerical errors are defined as follows

$$
\begin{aligned}
e_{s, \max } & =\max _{i j}\left|s\left(\mathbf{x}_{i j}\right)-w_{i j}\right| \approx\|s-w\|_{L^{\infty}}, \\
e_{s, 2} & =\sqrt{\sum_{i j}\left|s\left(\mathbf{x}_{i j}\right)-w_{i j}\right|^{2} \Delta x \Delta y} \approx\|s-w\|_{L^{2}} .
\end{aligned}
$$

Here, $w_{i j}$ is the numerical solution $w$ evaluated at the grid point $\left(x_{i}, y_{j}\right)=\mathbf{x}_{i j}$ whereas $s\left(\mathbf{x}_{i j}\right)$ is the exact solution for saturation. The errors for the pressure and the velocity are computed in a similar fashion. The order of accuracy is computed using the formula $\log _{2}\left(e_{\alpha}(h) / e_{\alpha}(h / 2)\right),(\alpha=2, \infty)$.

The exact solution, given above, has been carefully chosen to ensure that the coefficient matrices of the resulting linear algebraic systems are well conditioned. The simulations have been carried out with different values of the constant $C$ but the results presented below are for $C=0$. The errors between the exact and the numerical solutions for the saturation, pressure and velocity in the $L^{2}$ norm and the $L^{\infty}$ norm and the 
respective orders of accuracy are presented in Table 1. These have been computed at the final time $T=0.5$ with a time-step of 0.005 . We observe the following approximate order of accuracy in space.

$$
\|p-r\|_{L^{2}}=O\left(h^{2}\right) \quad \& \quad\|\mathbf{v}-\boldsymbol{z}\|_{L^{2}}=O\left(h^{2}\right) .
$$

Table 1 shows that for a given fixed time-step, the errors decrease with refinement of the spatial mesh. However, for larger time steps (larger than 0.05) we find (data not shown here) that the time discretization errors dominate the spatial discretization errors. This leads to an increase in the $L^{2}$ and $L^{\infty}$ error norms and a reduction in the order of accuracy. For example, the poor order of accuracy in the $L^{2}$ norm of the error in velocity seen in the last row of Table 1 has been found to improve significantly when a smaller time step is used for the entire simulation. The order of accuracy in the $L^{\infty}$ norm of the error in saturation, presented in the upper part of the last column of Table 1, can also be seen to reduce significantly with reduction in spatial grid size. This is because the numerical solution attains a high accuracy, to the order of $10^{-5}$, even at the largest spatial grid size used $(h=1 / 8)$. The error between the actual solution and the numerical solution does not improve much further and causes the loss in the order of accuracy.

Table 1: Error and order for saturation, pressure and velocity using the analytic solution at the final time $T=0.5$ with $\Delta t=0.005$.

\begin{tabular}{|c|c|c|c|c|c|}
\hline & $h$ & $\|s-w\|_{L^{2}}$ & Order & $\|s-w\|_{L^{\infty}}$ & Order \\
\hline \multirow{6}{*}{ 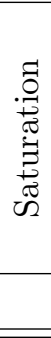 } & $1 / 8$ & $3.69 \mathrm{e}-5$ & - & $4.97 \mathrm{e}-5$ & - \\
\hline & $1 / 16$ & $1.36 \mathrm{e}-5$ & 1.442 & $5.38 \mathrm{e}-5$ & 0.891 \\
\hline & $1 / 32$ & $6.27 \mathrm{e}-6$ & 1.116 & $4.33 \mathrm{e}-5$ & 0.313 \\
\hline & $1 / 64$ & $3.07 \mathrm{e}-6$ & 1.027 & $4.08 \mathrm{e}-5$ & 0.087 \\
\hline & $1 / 128$ & $1.53 \mathrm{e}-6$ & 1.006 & $4.02 \mathrm{e}-5$ & 0.021 \\
\hline & $h$ & $\|p-r\|_{L^{2}}$ & Order & $\|p-r\|_{L^{\infty}}$ & Order \\
\hline \multirow{6}{*}{$\begin{array}{l}0 \\
0 \\
0 \\
0 \\
0 \\
0 \\
0 \\
D_{1}\end{array}$} & $1 / 8$ & $3.35 \mathrm{e}-3$ & - & $1.20 \mathrm{e}-2$ & - \\
\hline & $1 / 16$ & $1.04 \mathrm{e}-3$ & 1.688 & $3.92 \mathrm{e}-3$ & 1.611 \\
\hline & $1 / 32$ & $3.16 \mathrm{e}-4$ & 1.720 & $1.23 \mathrm{e}-3$ & 1.668 \\
\hline & $1 / 64$ & $9.36 \mathrm{e}-5$ & 1.754 & $3.73 \mathrm{e}-4$ & 1.725 \\
\hline & $1 / 128$ & $2.72 \mathrm{e}-5$ & 1.784 & $1.10 \mathrm{e}-4$ & 1.768 \\
\hline & $h$ & $\|\mathbf{v}-\mathbf{z}\|_{L^{2}}$ & Order & $\|\mathbf{v}-\mathbf{z}\|_{L^{\infty}}$ & Order \\
\hline \multirow{5}{*}{$\begin{array}{l}\stackrel{\overrightarrow{0}}{.0} \\
0 \\
\frac{0}{0} \\
p\end{array}$} & $1 / 8$ & $1.59 \mathrm{e}-3$ & - & $3.27 \mathrm{e}-3$ & - \\
\hline & $1 / 16$ & $4.13 \mathrm{e}-4$ & 1.943 & $1.51 \mathrm{e}-3$ & 1.118 \\
\hline & $1 / 32$ & $1.07 \mathrm{e}-4$ & 1.950 & $7.17 \mathrm{e}-4$ & 1.069 \\
\hline & $1 / 64$ & $2.82 \mathrm{e}-5$ & 1.923 & $3.50 \mathrm{e}-4$ & 1.036 \\
\hline & $1 / 128$ & $9.21 \mathrm{e}-6$ & 1.614 & $1.75 \mathrm{e}-4$ & 1.018 \\
\hline
\end{tabular}

The non-symmetric algebraic system obtained by assembling the stiffness matrix of the finite element weak formulation, given by eq. (41), and the symmetric but sparse algebraic system obtained by discretizing the transport equation, given by eq. (55), have been solved by a BICGstab iterative method with tolerance values in the range $10^{-6}-10^{-9}$ and without any preconditioners. Apart from achieving high accuracy, there is one more important significance of choosing such ultra-low values of tolerance, specifically for solving the transport equations. It has been observed that the BICGstab solver converges to a solution with a relative 


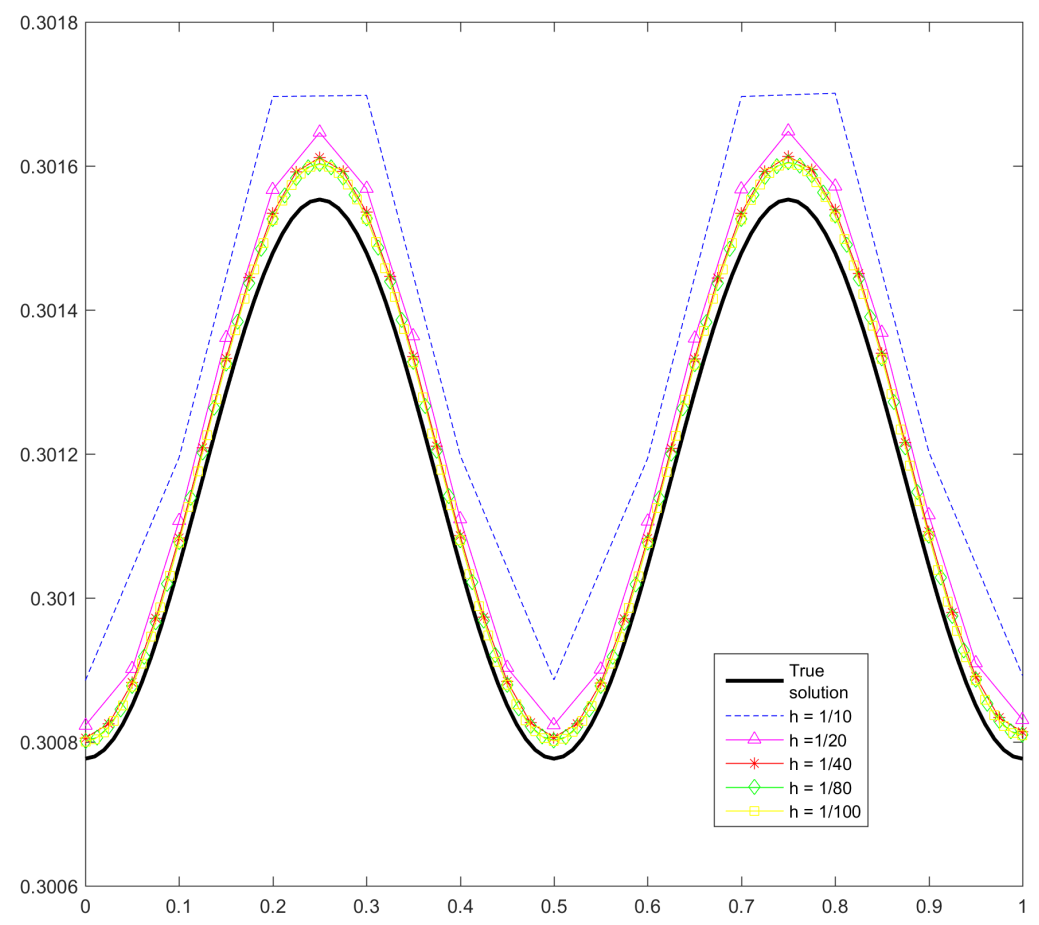

Figure 11: Comparison between the saturation profiles of the exact solution and the numerical solutions at various spatial resolutions. The profiles are plotted along the horizontal center line $(y=0.5)$ of the domain, $\Omega=[0,1]^{2}$ at $t=0.1$.

residual of about $10^{-6}$ within the first two iterations for even moderately fine spatial resolution of $h=1 / 32$. Hence, it becomes necessary to choose much lower levels of tolerance in order to compute the order of accuracy with respect to spatial discretization for the water saturation. However, it is worth pointing out that there is a delicate balance involved in such a choice. If the tolerance is reduced even further from $10^{-9}$, then the BICGstab iteration process may stagnate and fail to achieve the desired relative residuals. Although these observations were made in the context of the exact solution of waterflooding, these are also valid in the context of the transport equations for the polymer and the surfactant, given by eqs. (56) and (57).

In Fig. 11 we plot the water saturation profiles along the center line, $y=0.5$ at an intermediate time $t=0.1$. The true solution is plotted with the solid black line while the numerical solution obtained on a sequence of successively refined grid sizes, $h=1 / 10,1 / 20,1 / 40,1 / 80,1 / 100$ are plotted in different colors and line markers. This figure shows that the numerical solution monotonically improves with reduction in spatial grid size and converges toward the exact solution.

Table 2 shows the numerical errors and the order of accuracy for the saturation equation with respect to time for three choices of fixed spatial grid sizes, namely $h=1 / 16,1 / 32$ and $1 / 64$. The errors are computed at the final time $T=1$. The results confirm that at least a first-order convergence rate in time can be obtained using this method. This compares favorably with results obtained by others (see [71]) on similar types of problems. Additionally, we expect that with higher order time-stepping methods, the method will be able to preserve the accuracy and the expected second or third order convergence rates. It is also important to note that with a fixed time step, the accuracy of the method increases with decreasing spatial grid size which can also serve as a quick validation of the results presented in Table 1. Moreover, Table 2 shows a higher than expected convergence rate with respect to time. This is because the reduction in the error norm associated with a time step size refinement increases as the spatial grid size increases.

From Table 1 and Table 2 we can also infer that the method suffers from very minimal numerical diffusion. The $L^{2}$ error in the water saturation values lies in the range of $10^{-5}-10^{-7}$ when spatial grid sizes lie in 
Table 2: Error and rates for saturation with time step refinement, computed at $T=1$ using the analytical solution.

\begin{tabular}{cccccc}
\hline$h$ & $\Delta t$ & $\|s-w\|_{L^{2}}$ & Rate & $\|s-w\|_{L^{\infty}}$ & Rate \\
\hline \hline & $1 / 20$ & $1.57 \mathrm{e}-4$ & - & $1.00 \mathrm{e}-3$ & - \\
$\frac{1}{16}$ & $1 / 40$ & $3.58 \mathrm{e}-5$ & 1.670 & $3.36 \mathrm{e}-4$ & 1.588 \\
& $1 / 80$ & $8.49 \mathrm{e}-6$ & 1.625 & $1.10 \mathrm{e}-4$ & 1.610 \\
& $1 / 320$ & $5.51 \mathrm{e}-7$ & 1.464 & $1.41 \mathrm{e}-5$ & 1.462 \\
\hline \hline & $1 / 20$ & $6.82 \mathrm{e}-5$ & - & $4.20 \mathrm{e}-4$ & - \\
& $1 / 40$ & $1.99 \mathrm{e}-5$ & 1.297 & $1.76 \mathrm{e}-4$ & 1.253 \\
$\frac{1}{32}$ & $1 / 80$ & $5.75 \mathrm{e}-6$ & 1.319 & $7.14 \mathrm{e}-5$ & 1.302 \\
& $1 / 160$ & $1.69 \mathrm{e}-6$ & 1.260 & $2.99 \mathrm{e}-5$ & 1.253 \\
& $1 / 320$ & $4.73 \mathrm{e}-7$ & 1.299 & $1.22 \mathrm{e}-5$ & 1.296 \\
\hline \hline & $1 / 20$ & $4.91 \mathrm{e}-5$ & - & $3.12 \mathrm{e}-4$ & - \\
& $1 / 40$ & $1.62 \mathrm{e}-5$ & 1.077 & $1.37 \mathrm{e}-4$ & 1.184 \\
$\frac{1}{64}$ & $1 / 80$ & $5.08 \mathrm{e}-6$ & 1.168 & $6.22 \mathrm{e}-5$ & 1.144 \\
& $1 / 160$ & $1.56 \mathrm{e}-6$ & 1.165 & $2.78 \mathrm{e}-5$ & 1.162 \\
& $1 / 320$ & $4.50 \mathrm{e}-7$ & 1.247 & $1.17 \mathrm{e}-5$ & 1.244 \\
\hline \hline
\end{tabular}

the range of $10^{-2}-10^{-3}$. This indicates that the amount of numerical diffusion is negligible and does not dominate over the capillary pressure induced physical diffusion.

\subsection{Waterflood simulations}

\subsubsection{Waterflooding in a quarter five-spot geometry with one block inclusion in permeability}

Here we consider a special case of a porous medium that has a square block inclusion with lower permeability. We set the permeability of the shaded block (Fig. 12(a)) to be 1000 times smaller than in the rest of the domain. We simulate the displacement of the resident oil with a waterflood using a $30 \times 30$ grid on a quarter five-spot domain, $\Omega$. The water saturation contours inside the domain are compared at four different time levels during the displacement process (Fig. 13). We observe that the numerical method captures the region of discontinuity of the permeability very well and the results are in excellent qualitative agreement with similar simulation studies found in the literature (see [71]). It has been shown [72, 73] that the equations for immiscible two phase displacement and the equations for miscible displacement are mathematically equivalent under certain conditions. Chavent [72] showed that the global pressure formulation for immiscible displacement is identical in form to the miscible displacement equations. Yortsos [73] used a different nonphysical pressure variable to show the equivalence between two-phase immiscible displacement and singlephase miscible displacement in the presence of equilibrium adsorption. This analogy allows us to compare the evolution of saturation contours in our simulation to the evolution of concentration contours in an equivalent miscible flooding simulation [71]. 
Table 3: Simulation input data

\begin{tabular}{||lcc||}
\hline Model parameter & Symbol & Value \\
\hline \hline Spatial grid size & $h \times k$ & variable \\
Porosity & $\phi$ & 1 \\
Permeability & $\mathbf{K}$ & variable \\
Initial resident water saturation & $s_{0}^{\sigma 0}$ & 0.21 \\
Polymer injection concentration & $c_{0}$ & variable \\
Surfactant injection concentration & $\Gamma_{0}$ & variable \\
Oil viscosity & $\mu_{o}$ & 12.6 \\
Pure water viscosity & $\mu_{w}$ & 1.26 \\
Residual aqueous phase saturation & $s_{r a}^{\sigma 0}$ & 0.1 \\
Residual oleic phase saturation & $s_{r o}^{\sigma 0}$ & 0.2 \\
Critical capillary number of aqueous phase & $N_{c a o}$ & $10^{-5}$ \\
Critical capillary number of oleic phase & $N_{c o}$ & $10^{-5}$ \\
Parameters of capillary pressure relation [eq. (19)] & $\omega_{1}, \omega_{2}$ & $0.1,0.4$ \\
Injection rate & $Q$ & 200,50 \\
Time step size & $\Delta t$ & $1 / 50,1 / 100$ \\
\hline
\end{tabular}

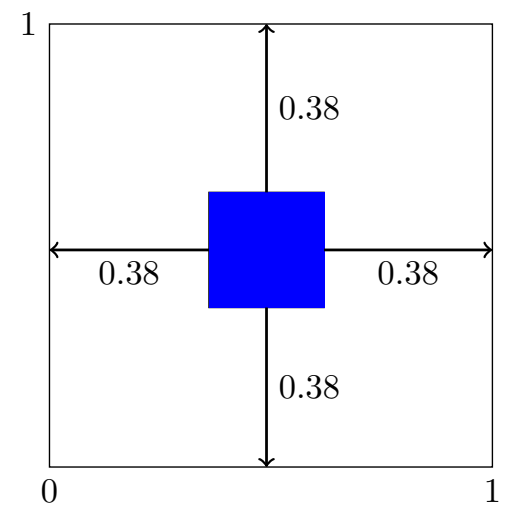

(a) Piecewise continuous permeability field with one block inclusion

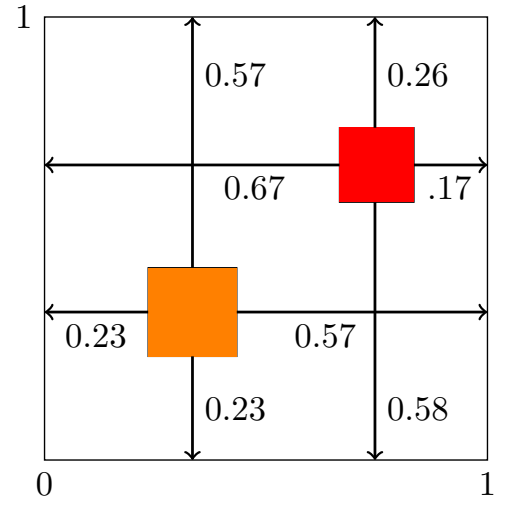

(b) Piecewise continuous permeability field with two block inclusions

Figure 12: Piecewise continuous permeability fields generated for qualitative validation studies

\subsubsection{Waterflooding in a quarter five-spot geometry with two block inclusions in permeability}

Next, we consider a permeability field with two rectangular block inclusions inside the domain. As before, the permeability of the shaded blocks (Fig. 12(b)) is set to be 1000 times smaller than in the rest of the domain. A waterflood is simulated on a $30 \times 30$ grid and the water saturation contours inside the domain at different points of time during the flooding process are compared (Fig. 14). We observe that the numerical method is successful in qualitatively capturing the discontinuity in the permeability data very well and the saturation contours appear to be in good qualitative agreement with what is expected based on physics. 
$\mathbf{t}=\mathbf{2 0 0}$

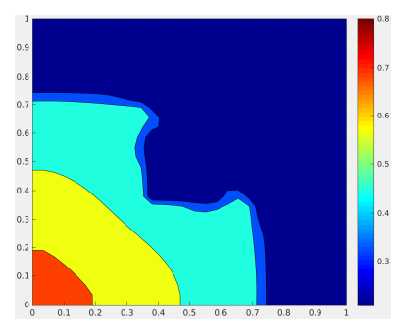

$\mathbf{t}=\mathbf{4 0 0}$

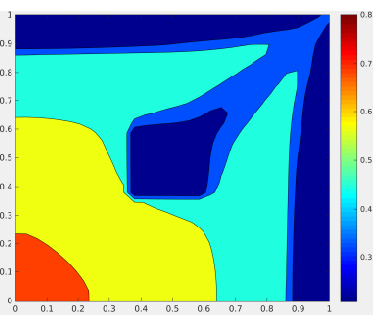

$\mathbf{t}=\mathbf{7 0 0}$

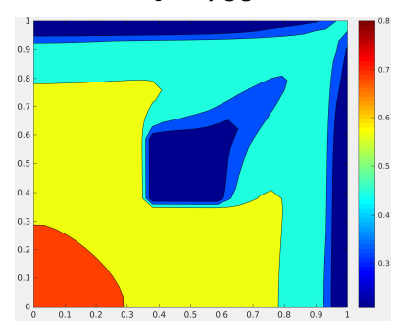

$\mathbf{t}=\mathbf{1 0 0 0}$

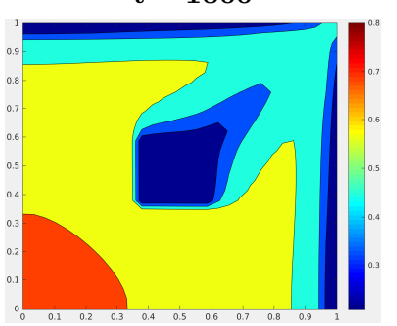

Figure 13: Saturation contours during a waterflood in a quarter five-spot reservoir with a spatial resolution of $30 \times 30$. The permeability field is piecewise continuous with a block inclusion at the center, as shown in Fig. 12(a). The contours are plotted at four different time levels, $t=200,400,700,1000$.

$\mathbf{t}=\mathbf{2 0 0}$

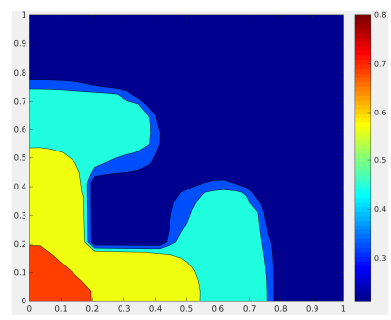

$\mathrm{t}=400$

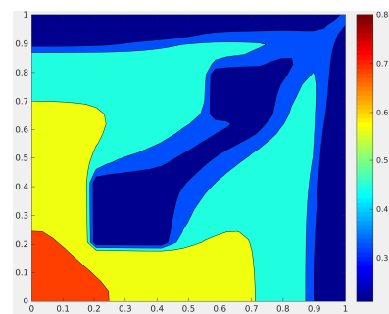

$\mathbf{t}=\mathbf{8 0 0}$

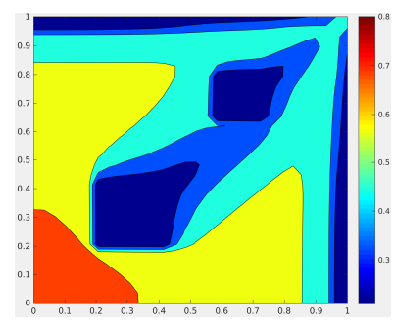

$\mathbf{t}=\mathbf{1 2 0 0}$

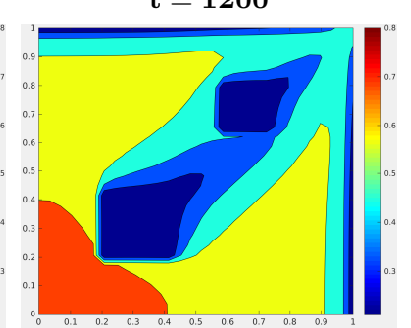

Figure 14: Saturation contours during a waterflood in a quarter five-spot reservoir with a spatial resolution of $30 \times 30$. The permeability field is piecewise continuous with two block inclusions, as shown in Fig. 12(b). The contours are plotted at four different time levels, $t=200,400,800,1200$.

\subsubsection{Waterflooding in a rectilinear geometry with a heterogeneous permeability field}

In this simulation we consider the rectilinear flow reservoir design, as shown in Fig. 3(b), in which the left boundary is treated as an injection well (line source) and the right boundary is treated as a production well (line sink). We consider a heterogeneous porous medium and choose the permeability function $\mathbf{K}(\mathbf{x})$ as given in [74]. The permeability function is given by

$$
\mathbf{K}(\mathbf{x})=50\left[0.5\left(1-10^{-7}\right)(\sin (6 \pi \cos x) \cos (4 \pi \sin (3 y))-1)+1\right],
$$

and it is shown in the leftmost plot of Fig. 15 on a $60 \times 60$ spatial grid. As shown in Fig. 15, the more mobile fluid (water) moves along the pathways formed by inter-connected zones of higher permeability. These heterogeneity driven finger formations are in agreement with the results obtained in [74] and provide further validation of our method. It is clear from the saturation contours that the regions of higher water saturation (shown in red) are trailing behind the rapidly advancing fingers. This causes premature water breakthrough (production well starts to produce water), much before a significant amount of the residual oil has been pushed out. One of the important uses of adding polymer to the displacing phase is to inhibit the formation and growth of these fingers at an early stage by stabilizing the initial oil-water interface. This effect has been studied in more detail in one of the following simulation studies (see Fig. 29).

\subsubsection{Waterflooding in a rectilinear geometry with a channelized permeability field}

We again use a rectilinear flow domain for this study. We consider a heterogeneous porous medium and choose $\mathbf{K}(\mathbf{x})$ as in [75] in the shape of a region of high permeability that meanders from the left to the right. 
Permeability

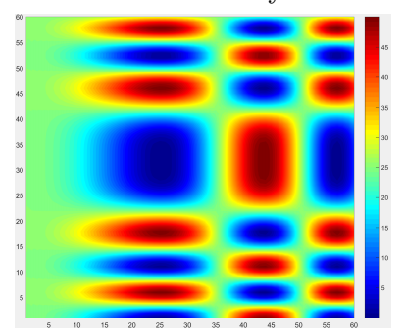

$\mathbf{t}=60$

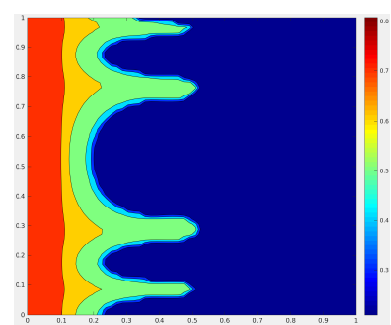

$\mathbf{t}=\mathbf{1 0 0}$

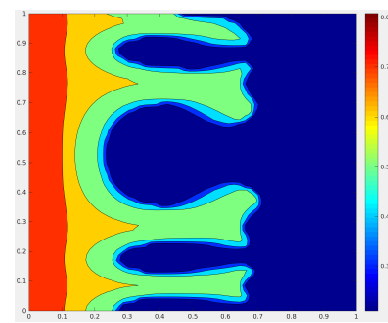

$\mathbf{t}=\mathbf{1 6 0}$

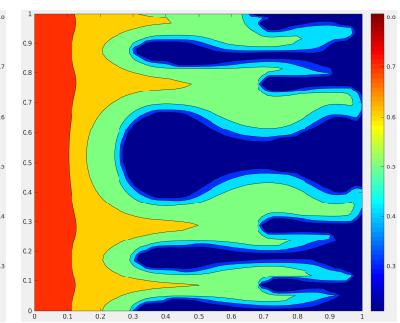

Figure 15: Saturation contours during a waterflood in a rectilinear geometry with $60 \times 60$ spatial resolution at three different time levels, $t=60,100,160$. The heterogeneous permeability field from [74] (plotted in the leftmost figure) has been used. In the permeability plot, the red regions represent higher permeability while the blue regions represent lower permeability.

The permeability function is given by

$$
\mathbf{K}(\mathbf{x})=\max \left[\exp \left(-\left(\frac{y-0.5-0.1 \sin (10 x)}{0.1}\right)^{2}\right), 0.01\right],
$$

and it is shown in the leftmost plot of Fig. 16 on a $60 \times 60$ spatial grid. The water saturation contours plotted at three different time levels are in good agreement with those in [75] and provide additional validation of the method. Moreover, this simulation study demonstrates the ability of the model and the method to accurately capture flow behavior in highly channelized reservoirs or in rocks with natural faults and cracks.
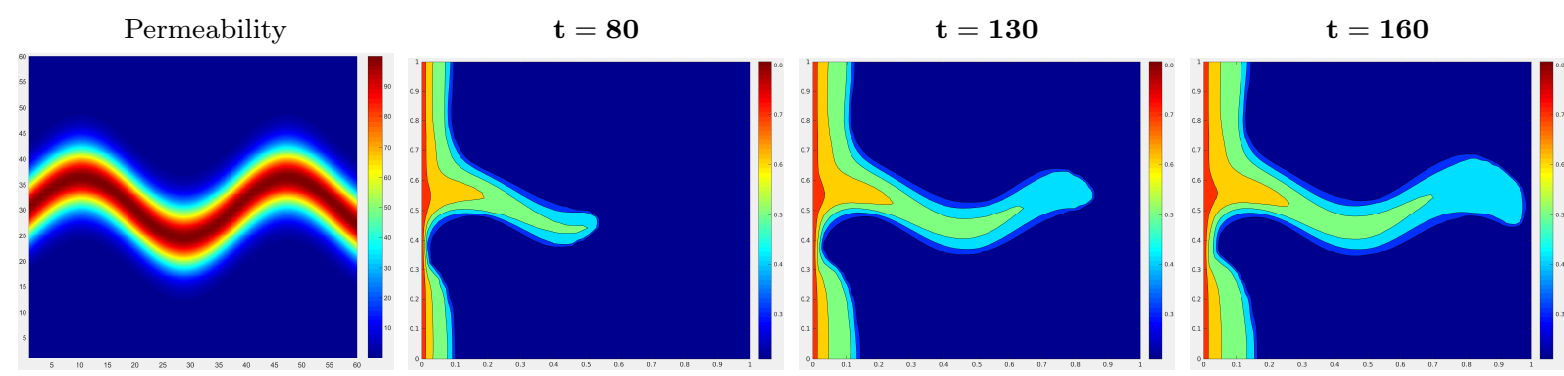

Figure 16: Saturation contours during a waterflood in a rectilinear geometry with $60 \times 60$ spatial resolution at three different time levels, $t=80,130,160$. The permeability field from [75] (plotted in the leftmost figure), which represents a single crack porous medium, has been used. In the permeability plot, the red regions represent higher permeability while the blue regions represent lower permeability.

\subsubsection{Waterflooding in a quarter five-spot geometry with the Upper-Ness (SPE10) perme- ability field}

In this simulation we use a $30 \times 30$ block of permeability field data from the Upper-Ness region of the SPE10 benchmark problem dataset (see [76]). The original dataset is described on a regular Cartesian grid with $60 \times 220 \times 85(1,122,000)$ cells. The model consists of two types of formations: a shallow-marine Tarbert formation in the top 35 layers with relatively smooth permeability, and a fluvial Upper-Ness permeability in the bottom 50 layers. Both formations (Fig. 17) are characterized by large permeability variations $(8$ to 12 orders of magnitude), but are different qualitatively. The Upper-Ness formation has more rapid variability 
while the Tarbert formation has a higher contrast in the permeability values. In this work, we present results obtained using both types of permeability field data.

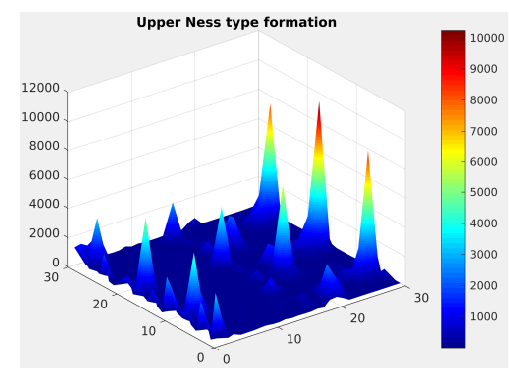

(a) Upper-Ness type permeability

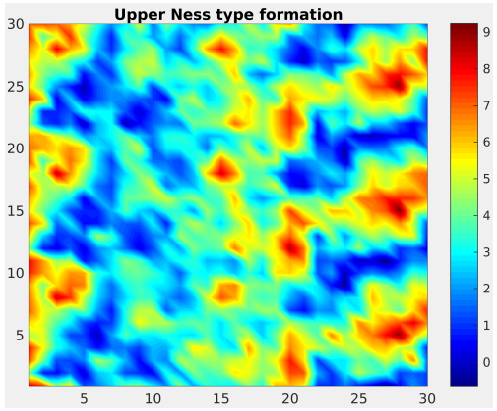

(c) Logarithmic permeability plot

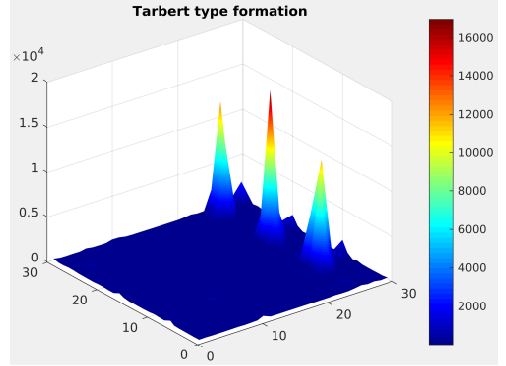

(b) Tarbert type permeability

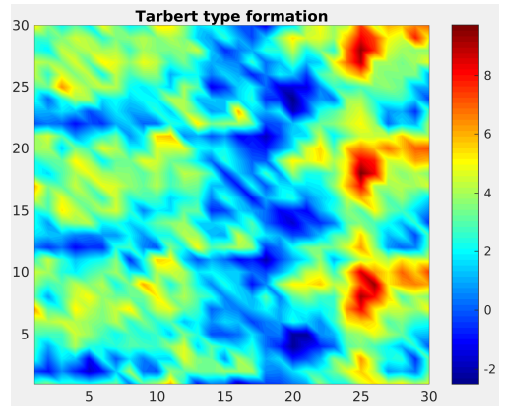

(d) Logarithmic permeability plot

Figure 17: Permeability plots from the SPE10 benchmark dataset on a $30 \times 30$ grid.
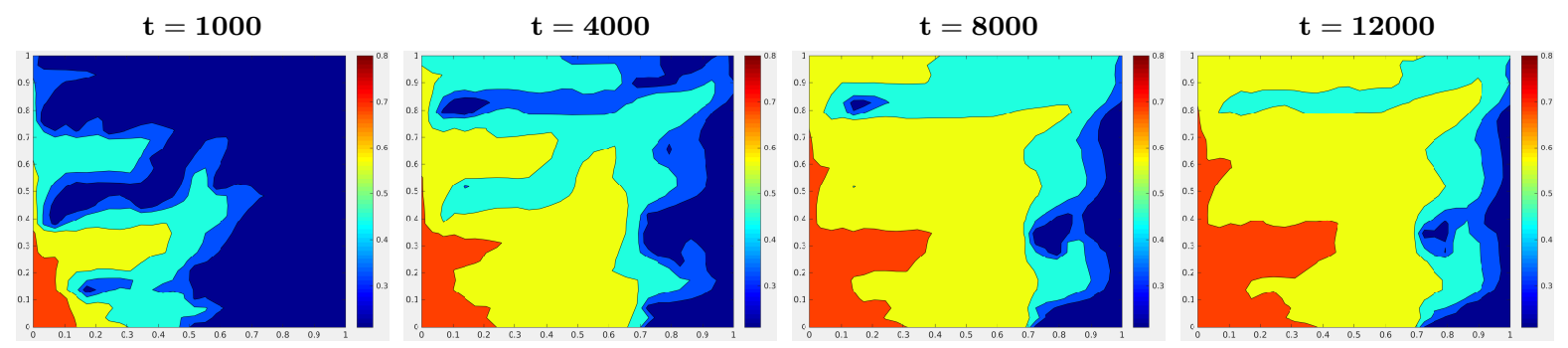

Figure 18: Saturation contours during a waterflood in a quarter five-spot reservoir with $30 \times 30$ spatial resolution. The permeability field is of Upper-Ness formation type (see Fig. 17(a) and Fig. 17(c)). The contours are plotted at four different time levels, $t=1000,4000,8000,12000$.

The simulation results are shown in Fig. 18. As expected, the highly heterogeneous rock matrix leads to development of undesirable finger formations while the high viscosity ratio $\left(M_{\mu}=\mu_{o} / \mu_{w}=10\right)$ between the displaced and displacing phase leads to the growth of these viscous fingers. We observe that the displacing phase progresses towards the production well (located at $(x, y)=(1,1)$ ) by sweeping through the regions of higher permeability values. The method captures the expected features of fluid flow through the regions of high and low permeability very well and the fronts are well resolved. This provides qualitative validation of the model and the numerical method as it is able to capture the expected physical phenomenon like viscous finger formations in fully developed flows. 


\subsubsection{Study of grid orientation effects}

Many of these waterflooding simulations involve adverse viscosity ratio $\left(M_{\mu}=\mu_{o} / \mu_{w}\right)$ displacement as the displacing aqueous phase usually flows more easily through the porous medium than the displaced oil phase. For these flows, numerical methods can suffer from grid orientation effects depending on whether the flow is parallel or diagonal to the computational grid. This phenomenon was first reported in [77]. It was observed there that quarter five spot simulations have two different principal axes of symmetry and accordingly the simulation grid can be aligned in two different ways. At an adverse viscosity ratio, the simulations were found to converge to different solutions on these two different grid alignments. There is a rich literature dedicated towards understanding this phenomenon and developing approaches to alleviate these grid orientation effects. We refer to [78] for a comprehensive summary.

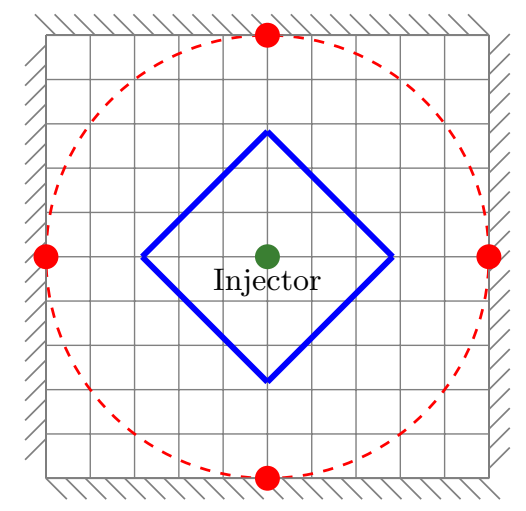

(a) Parallel flow

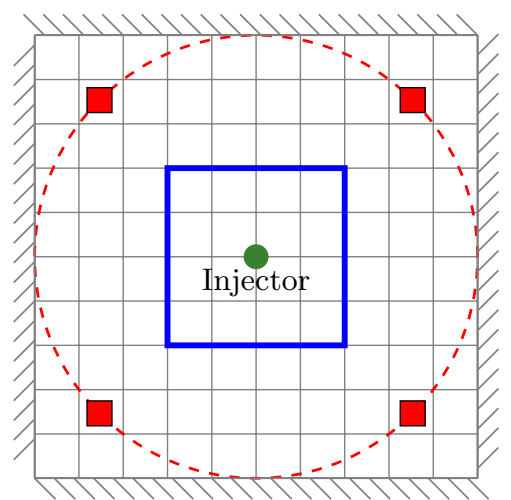

(b) Diagonal flow

Figure 19: Simulation domain designs for study of grid orientation effects. The flow direction is parallel to the computational grid in part (a) and diagonal to the computational grid in part (b).

In the next set of simulations we investigate if our method suffers from significant grid orientation effects. For this purpose we consider two special five-spot flow geometries, as shown in Fig. 19. We consider a square domain with an injection well or source at the center and four production wells or sinks placed in two different configurations. In Fig. 19(a) the positions of the producers ensure that the effective flow is along the computational grid, while in Fig. 19(b) the producers are placed in such a way that the effective flow is diagonal to the computational grid. In order to make these two configurations equivalent, we choose the boundary of a square as the initial position of the interface separating the displacing phase from the displaced phase. In both the configurations, each vertex of this square is located on the line connecting the injector with one of the producers. The producers are also placed in such a way that they are at the same distance from the source in both the cases. Effectively, these are two equivalent configurations for a five-spot flow. The only difference is in the alignment of the computational grid.

In Fig. 20 we compare the parallel grid solutions obtained using the geometry in Fig. 19(a) with the diagonal grid solutions obtained using the geometry in Fig. 19(b). A homogeneous permeability field is considered. The source strength is kept fixed at $Q=10$ with a spatial resolution of $40 \times 40$ and a time step size of $\Delta t=1 / 80$. The simulations were done at a viscosity ratio of $M_{\mu}=20$ in order to trigger any grid alignment effects induced by the adverse viscosity ratio. The water saturation contours at two different times during the simulation on both the grids are presented in Fig. 20. The parallel flood contours are rotated counterclockwise by an angle of $45^{\circ}$ to facilitate the comparison with the diagonal flood contours. It can be clearly seen that there are very minor differences in the features of the contours but they are qualitatively very similar. Some of the minor differences can be attributed to a graphical issue that comes up while rotating the image counterclockwise by $45^{\circ}$ using the Image Processing toolbox of MATLAB. Rotating the 


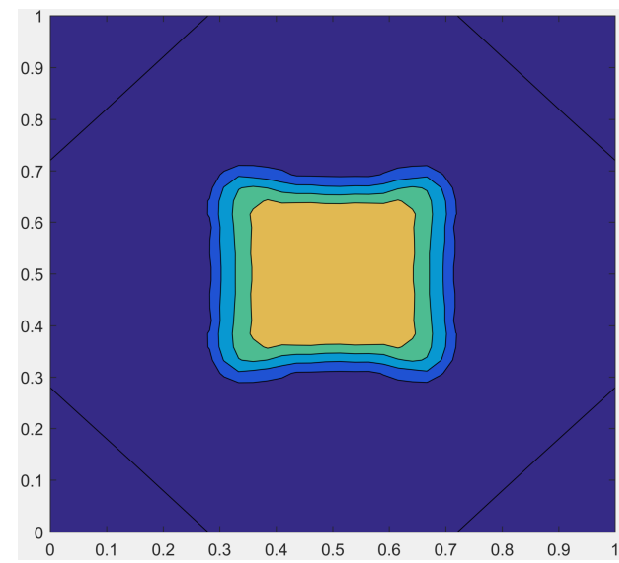

(a) $t=200$, parallel flood

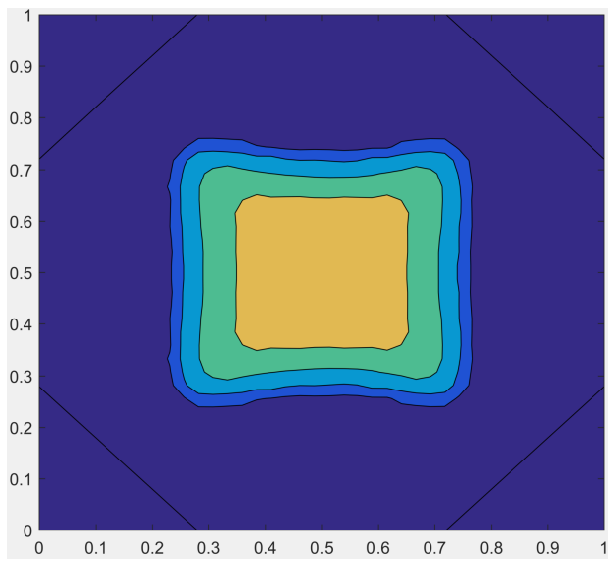

(c) $t=400$, parallel flood

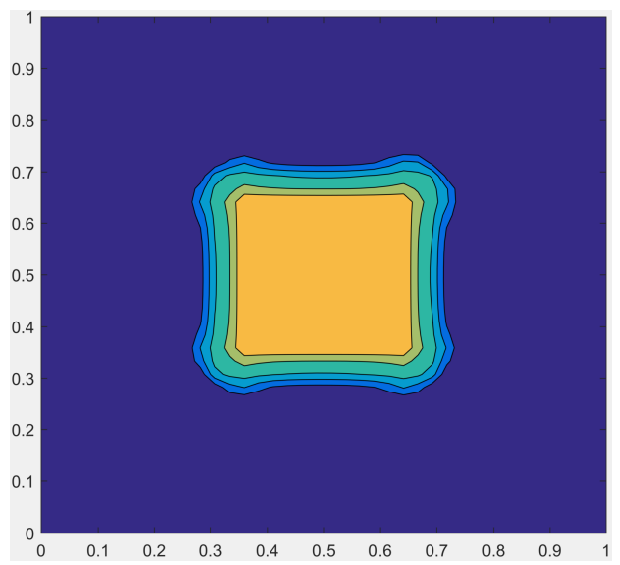

(b) $t=200$, diagonal flood

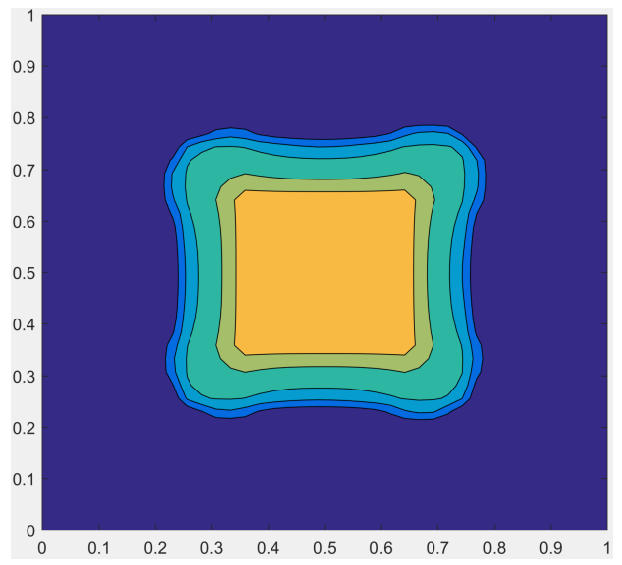

(d) $t=400$, diagonal flood

Figure 20: Comparison of water saturation contours for parallel and diagonal flow simulations in a fivespot geometry with a spatial resolution of $40 \times 40$ to study the grid orientation effects. The left column corresponds to the parallel flow and the right column corresponds to the diagonal flow simulations at two different time levels, $t=200$ and $t=400$. Some of the minor differences are explained in the discussion in section 4.2 .6 .

matrix of water saturation values in $\Omega$ by $n \pi / 2$ where $n$ is a non-integral value produces a new matrix of water saturation values. In this process, some data points are lost while some new data points are introduced which need to be evaluated by a linear or spline interpolation process. This adjustment causes some of the minor changes observed in the parallel flood figures. For instance, four fine black lines lying diagonally across the four corners of the domain are introduced in the contour map. Also, the rotated data matrix is plotted using 4 contour lines as opposed to the 5 contour lines used in the diagonal flood figures (which are not rotated). In spite of these graphical changes, the development of the saturation contours can be seen to follow a very similar trend in both the grid configurations. This leads us to conclude that our method suffers from negligible grid orientation effects and hence can be used reliably as a predictive numerical model. 


\subsection{Polymer flood simulations}

The displacement processes involved in tertiary oil recovery methods like polymer flooding and SP flooding are usually unstable to begin with. Hence one of the fundamental fluid dynamical mechanisms that is employed to improve oil recovery is to delay and slow down the development of these instabilities that lead to development of these nonlinear fingers since there is always a finite reservoir area that needs to be swept. The growth of these initial disturbances can be controlled by various means including the use of optimal values of the viscosity ratio $[79,80,81]$. The following set of simulations has been performed to compare the flow characteristics of a waterflood with that of a polymer flood.

\subsubsection{Polymer flooding in a quarter five-spot domain with the Tarbert permeability field}

In this simulation, we use the Tarbert permeability field data, shown in Fig. 17(b) and Fig. 17(d) on a $30 \times 30$ grid. We compare the flow patterns of two different waterflood scenarios with that of two different polymer floods. As shown in Fig. 21, the change in viscosity ratio from $M_{\mu}=\mu_{o} / \mu_{w}=10$ and $M_{\mu}=5$ in the waterfloods to $M_{\mu}=\mu_{o} / \mu_{a}=1.82$ and $M_{\mu}=1.25$ in the two polymer floods causes a marked change in the sweeping pattern of the process. We see that in the case of waterfloods, the water phase advances through the heterogeneous formation much faster compared to the polymer floods. This is due to the fact that pure water being less viscous is more mobile. On the other hand, the polymer floods are able to achieve a much more efficient sweep of the domain. Figure 21 shows that the higher water saturation contours (plotted in yellow and red colors) have advanced further in the polymer floods than in the waterfloods, at the same time point. Although this makes polymer flooding a slower recovery process compared to waterflooding, the net oil recovery can be substantially more in polymer flood. This claim is supported by the last row of plots in Fig. 21 where the saturation contours have been plotted at the time of water breakthrough in each flooding process. The polymer flood results clearly show that a larger area has been swept and also the higher water saturation levels (yellow contour) have penetrated deeper into the domain compared to the waterflood results. Between the first two columns, both showing waterfloods, the difference in mobility ratios has a significant effect in controlling the growth of the most advanced saturation contour. This is evident in the fact that the waterflood with a mobility ratio of $M_{\mu}=5$ takes longer time $(t=2418)$ for water breakthrough than the time taken $(t=1673)$ by the waterflood with a higher mobility ratio of $M_{\mu}=10$. However, there is little difference in the water saturation contours between the two polymer floods (see Columns 3 and 4 in Fig. 21) because the mobility ratios $\left(M_{\mu}=1.82\right.$ and $\left.M_{\mu}=1.25\right)$ are very close.

\subsubsection{Grid refinement study with polymer flooding}

In the next simulation of polymer flooding in a quarter five-spot geometry, we refine the grid over three steps $-15 \times 15,30 \times 30$ and $60 \times 60$. The domain has two rectangular inclusions in the permeability field such that the permeability of the shaded blocks (see Fig. 12(b)) is 1000 times smaller than that in the rest of the domain. The viscosity ratio is kept fixed at $M_{\mu}=1.67$. The water saturation contours for the polymer flooding process with three different grid sizes are given in Fig. 22. We observe that even over the wide range of grid sizes the evolution of the fronts near the region of discontinuous permeability is captured accurately. The details in the high gradient region of the saturation contours become more prominent with the reduction in mesh size due to the additional computational points added to the domain. The qualitative agreement between the saturation contours at the same time levels but with different grid sizes provide further validation of the numerical convergence of the method.

\subsection{Surfactant-polymer flood simulations}

In the following simulations we investigate the effect of using surfactant as a chemical flooding agent. This is done by comparing the effectiveness of surfactant and SP floods as compared to polymer flood and waterflood on the basis of the change in mobility ratio, $M_{\lambda}$ values. The goals of these simulations are: (a) to demonstrate the robustness of the method in performing simulations of various different types of chemical 

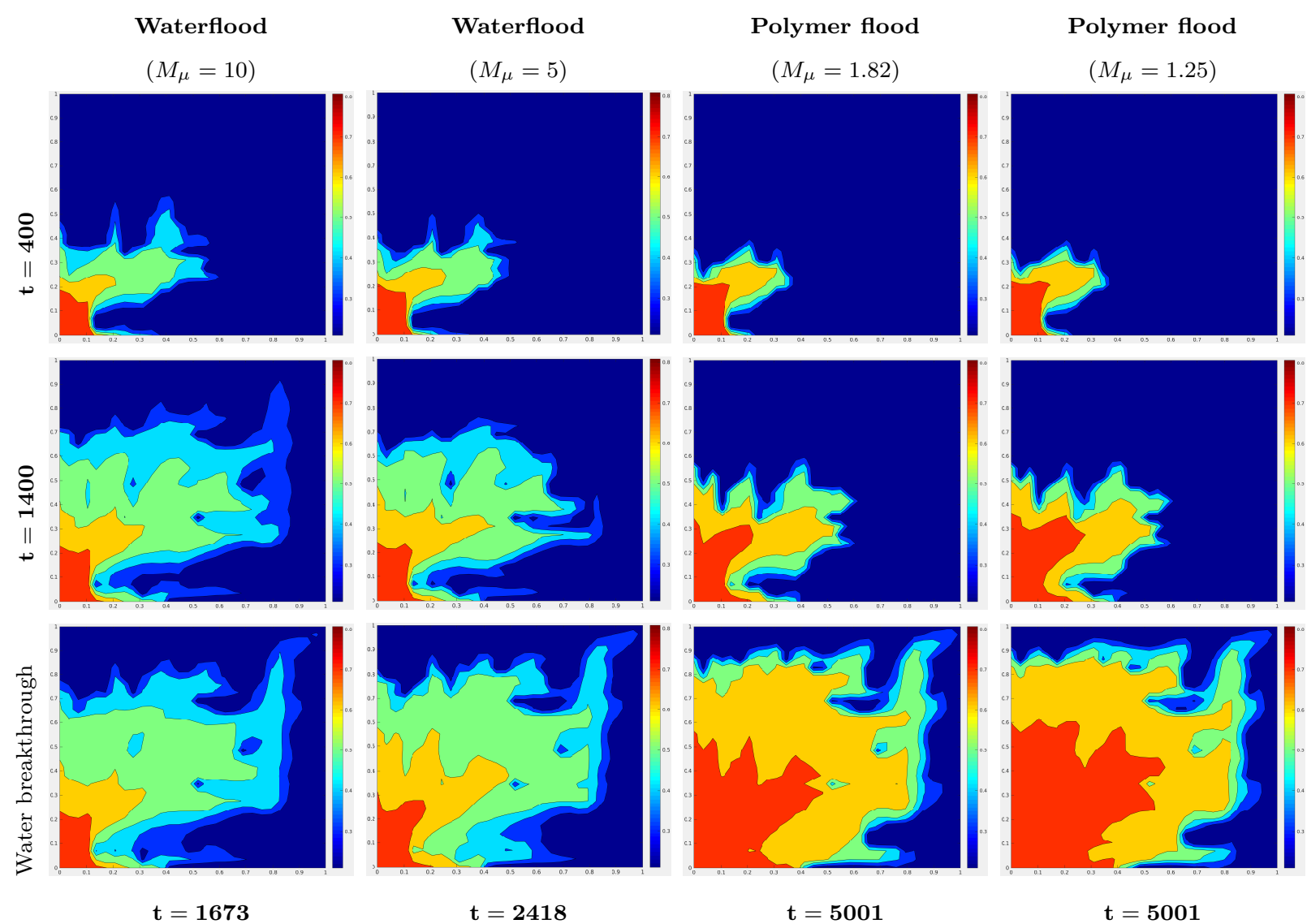

Figure 21: Saturation contours comparing waterfloods with polymer floods in a quarter five-spot reservoir with spatial resolution of $30 \times 30$. The simulations were carried out on the Tarbert formation type permeability field (see Fig. 17(b)). The first two columns correspond to waterfloods with viscosity ratios of $M_{\mu}=10$ and $M_{\mu}=5$ while the third and the fourth columns correspond to polymer floods with viscosity ratios given by $M_{\mu}=1.82$ and $M_{\mu}=1.25$ respectively. The contours are plotted at three different time levels, $t=400,1400$ and water breakthrough. The $t$ values below the last row of plots indicate the time levels at which water breakthrough occurs in each flood.

flooding processes and (b) to compare the effect of these different flooding schemes on the oil recovery, the sweeping efficiency, and on controlling the growth of fingers.

\subsubsection{Surfactant-polymer flooding in a quarter five-spot geometry}

The first two sets of simulations for surfactant-polymer flooding are performed in a quarter five-spot reservoir fitted with a $32 \times 32$ grid. A multiscale, stationary, isotropic Gaussian permeability field (Fig. 23(a)) generated using the method outlined in Section 3.4 has been used to construct a scalar, log-normal permeability field (Fig. 23(b)) with $k_{0}=3.44253, \varpi=1.0\left(C V_{k}=0.5\right)$.

The first set of simulations, presented in Fig. 24, provides a comparison of water saturation surface plots between four different types of flooding schemes at three different time levels. The first column represents waterflooding $\left(M_{\lambda}=2.89\right)$, the second represents surfactant flooding $\left(M_{\lambda}=3.12\right)$, the third and fourth columns represent two different schemes of SP flooding $\left(M_{\lambda}=3.06\right.$ and $\left.M_{\lambda}=1.96\right)$ which differ only in the amount of polymer present in the displacing phase. In Fig. 25, we compare the surfactant concentration 


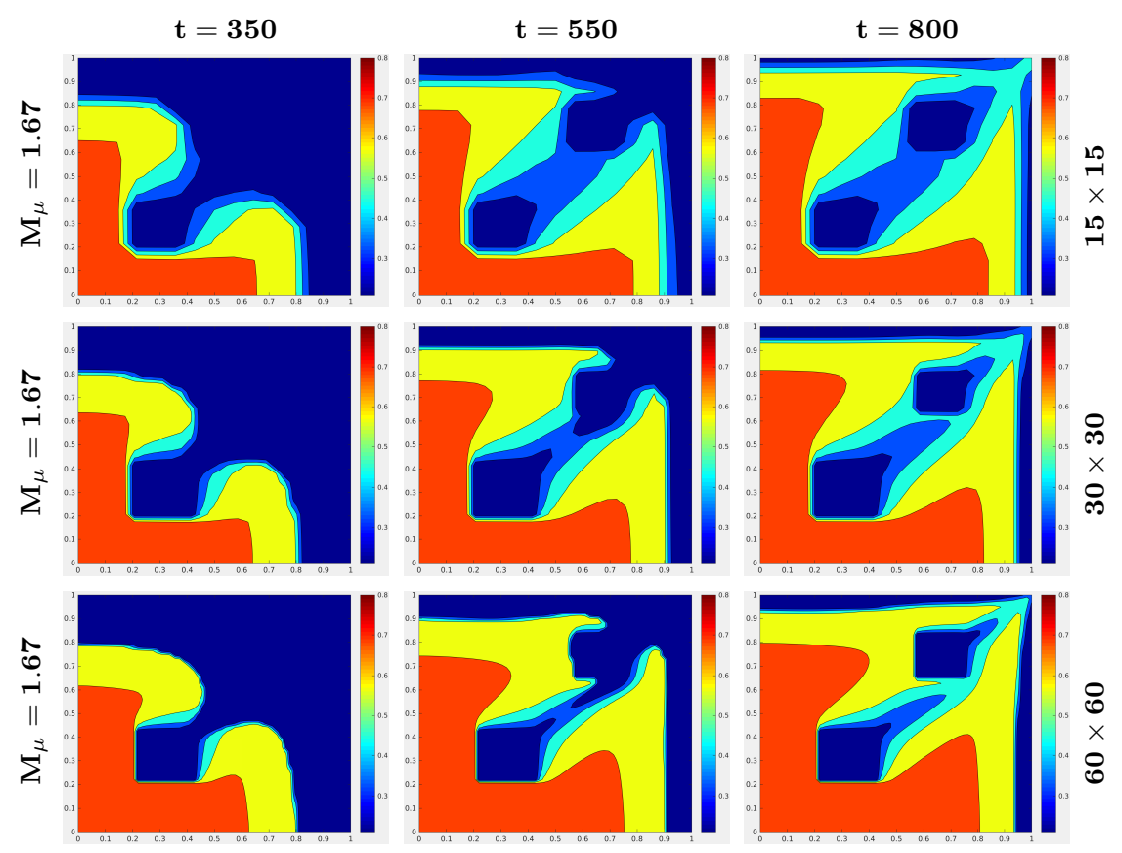

Figure 22: Water saturation contours during a polymer flood (viscosity ratio, $M_{\mu}=1.67$ ) in a quarter fivespot geometry with a piecewise continuous permeability field containing two block inclusions (see Fig. 12(b)). The contours have been plotted at three different time levels, $t=350,550,800$. The simulations were performed on three different spatial resolutions : Row $1-15 \times 15$, Row $2-30 \times 30$ and Row $3-60 \times 60$.

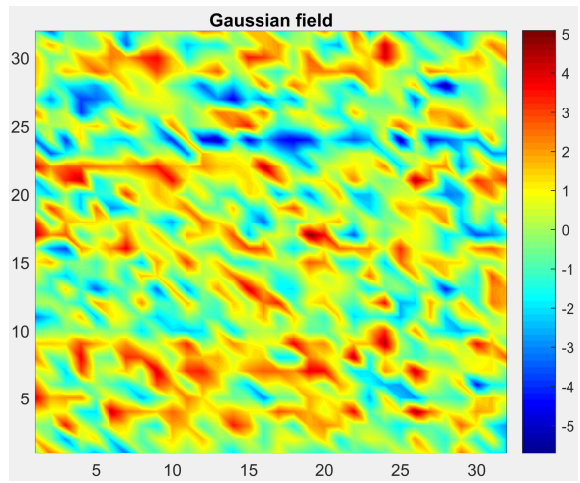

(a) Gaussian field

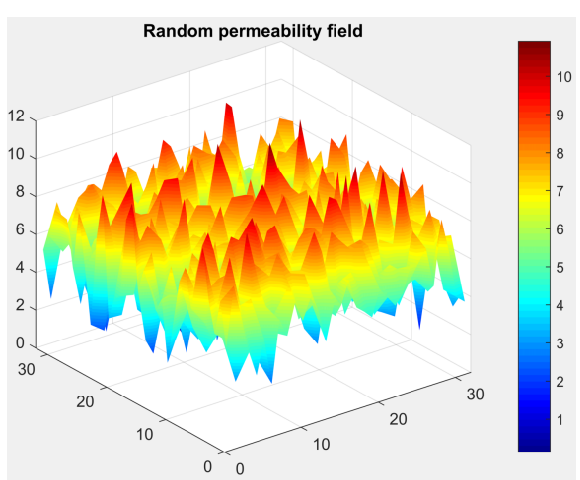

(b) Logarithmic plot of the permeability field

Figure 23: Multi-scale, stationary, isotropic Gaussian distribution [shown in (a)] which is used to generate a scalar log-normal permeability field [shown in (b)] of spatial resolution $32 \times 32$.

surface plots and the polymer concentration surface plots for these floods. Specifically, the first and the second columns show the surfactant concentration plots during the surfactant flood and the second SP flood $\left(M_{\lambda}=1.96\right)$ while the third and fourth columns present the polymer concentration surface plots between the two different SP floods.

The difference in the surface plots between the waterflood and the surfactant flood simulations is explained by the combined effect of the surfactant on the interfacial tension and the relative permeability of the aqueous 


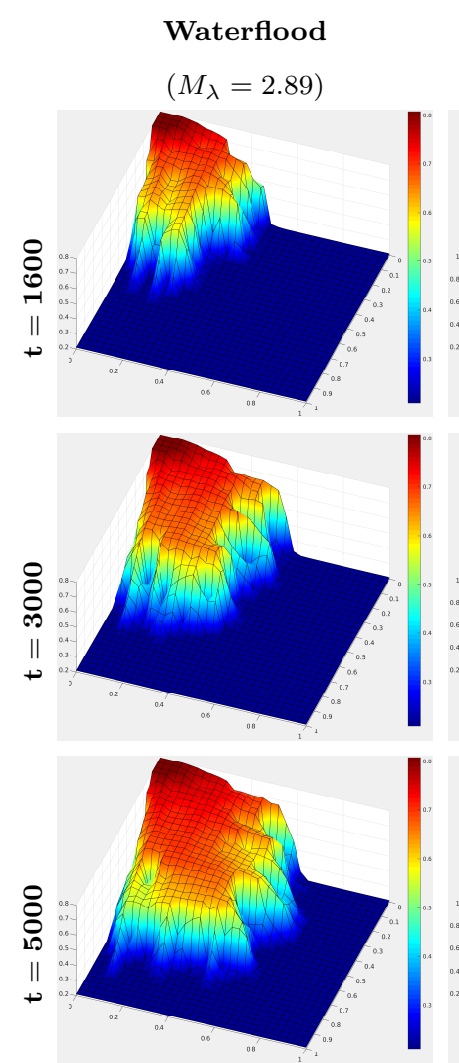

Surfactant flood

$\left(M_{\lambda}=3.12, \Gamma_{0}=0.05\right)$
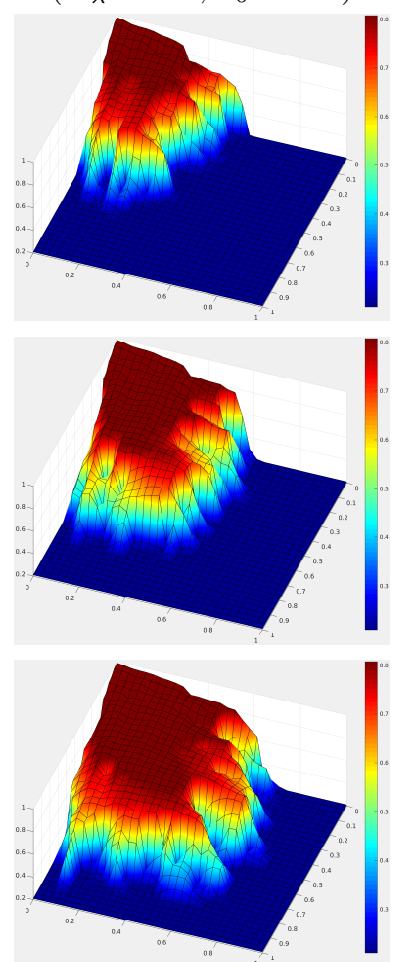

SP flood

$\left(M_{\lambda}=3.06, \Gamma_{0}=0.05\right)$
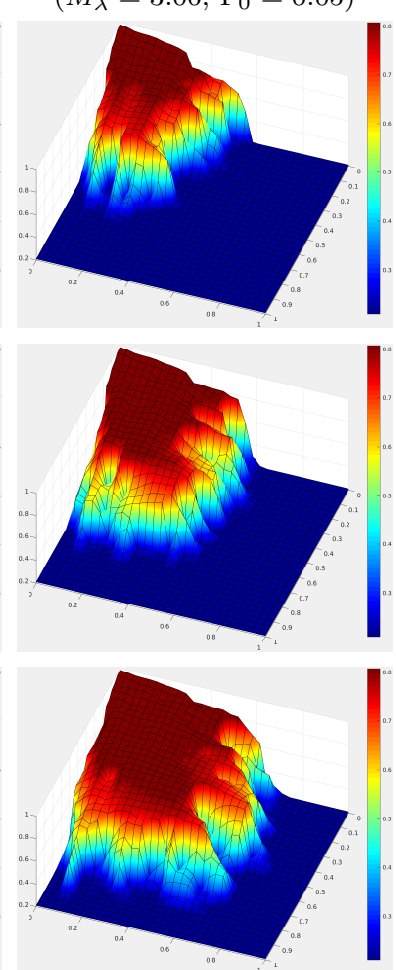

SP flood

$\left(M_{\lambda}=1.96, \Gamma_{0}=0.05\right)$
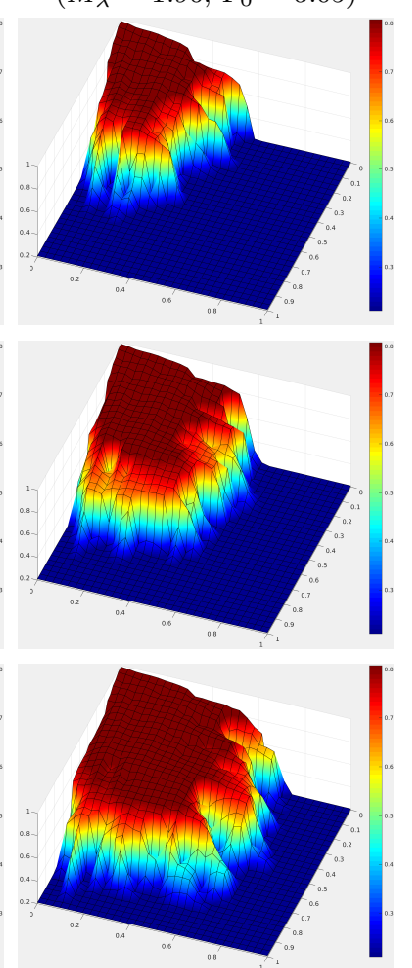

Figure 24: Comparison of water saturation surface plots in a quarter five-spot reservoir with spatial resolution of $32 \times 32$ during four different floods - waterflood, surfactant flood and two SP floods. The mobility ratios are $M_{\lambda}=2.89,3.12,3.06$ and 1.96 , respectively for the four floods. $\Gamma_{0}=0$ for the waterflood and $\Gamma_{0}=0.05$ for the other three floods. The permeability field used is given in Fig. 23. The surface plots are shown at three different time levels, $t=1600,3000,5000$.

phase. This allows more resident oil to be swept out as seen in the first two columns. We see that the regions with higher water saturation values (i.e. regions where most of the oil has been pushed out), indicated in the darker shades of red, have advanced further into the domain for the surfactant flood than for the waterflood at comparable times. There is not much difference between the saturation surface plots of the surfactant flood (2nd column) and the first SP flood (3rd column), in spite of the fact that polymer is added to the system in the SP flooding. This is because, the addition of polymer does not cause an appreciable change in the mobility ratio $\left(M_{\lambda}=3.12\right.$ and $M_{\lambda}=3.06$ respectively) between these two flooding schemes. Hence these two flooding schemes produce very similar results and this serves as an additional validation of the robustness of the model. The difference in the saturation surface plots between the two SP floods is primarily observable in the features near the advancing fronts (i.e. near the edges of the surface plots). The higher water saturation values can be seen to have spread wider in the second SP flood (fourth column) than in any of the other floods. This means that the gradient of the water saturation is the largest in the second SP flood as the frontal region is spread over the thinnest area among all the four floods. Even in Fig. 25, the surfactant and polymer concentration surface plots of the second SP flood, as seen in the second and fourth columns respectively, have a higher gradient near the frontal region as compared to the surfactant flood or the first SP flood. This is expected as the combination of polymer and surfactant used in this particular flood gives rise to the lowest mobility ratio $\left(M_{\lambda}=1.96\right)$. As a result, the progress of the finger formations 

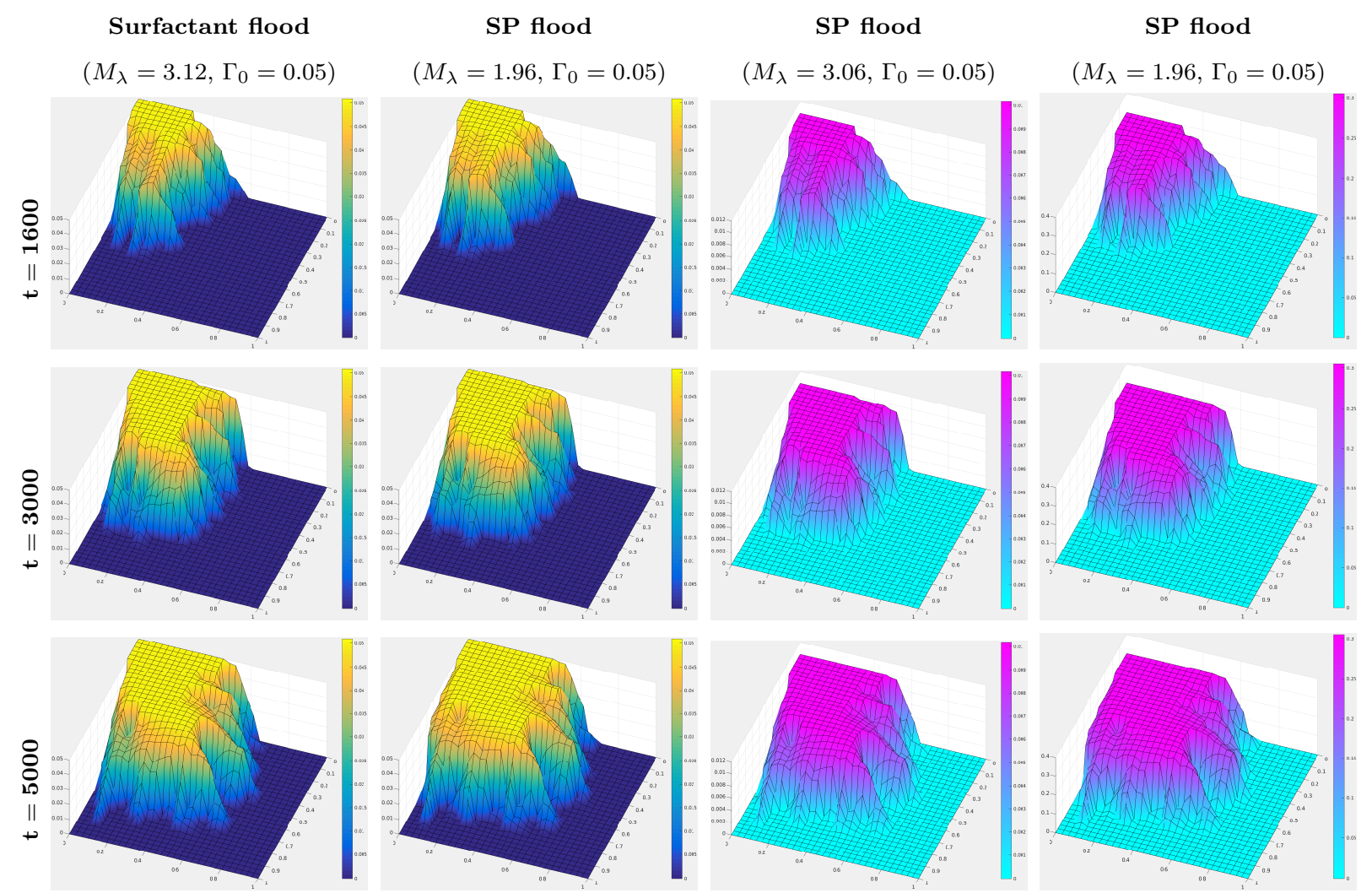

Figure 25: Comparison of surfactant (Columns $1 \& 2$ ) and polymer (Columns 3 \& 4) concentration surface plots in a quarter five-spot reservoir with spatial resolution of $32 \times 32$ during three different floods. The mobility ratio $M_{\lambda}=3.12,1.96$ for the first two columns while $M_{\lambda}=3.06,1.96$ for the third and fourth columns, respectively. $\Gamma_{0}=0.05$ is fixed for all the simulations. The permeability field given in Fig. 23 has been used. The surface plots are shown at three different time levels, $t=1600,3000,5000$.

is arrested to a higher extent and also a higher percentage of the resident oil is mobilized.

Some of the effects, discussed above, seem to be marginally observable in the surface plots, but the differences in the oil recovery efficiency and the change in the water cut at the production well with time, as presented in Fig. 26, strongly support these claims. The oil recovery efficiency, $E_{R}$ (in \%) is defined as the fraction of resident oil that is recovered in a flooding process. Figure 26(a) shows the recovery efficiency of four types of chemical flooding schemes with different mobility ratios: (a) waterflood $\left(M_{\lambda}=14.43\right)$, (b) surfactant flood $\left(M_{\lambda}=14.77\right)$, (c) SP flood $\left(M_{\lambda}=2.35\right)$ and (d) SP flood $\left(M_{\lambda}=2.98\right)$. The large drop in mobility ratios between the first two $\left(M_{\lambda}=14.43\right.$ and 14.77$)$ and the last two $\left(M_{\lambda}=2.35\right.$ and 2.98$)$ floods is used to enhance the difference in their efficiencies. Fig. 26(b) shows the time dependence of the water cut or the aqueous phase saturation $\left(s_{a}\right)$ at the production well for these four floods. As expected, in Fig. 26(a) we see that the oil recovery efficiency increases with the decrease in the mobility ratio of the scheme. The curves coincide initially and start to diverge as soon as water breakthrough is achieved in each flood. The respective breakthrough times are directly influenced by the mobility ratio of each flood, thus causing the flooding scheme with a higher $M_{\lambda}$ to breakthrough earlier. In Fig. 26(b), the water cut curves follow the same trend. The water cut is constant and the same for each flood until breakthrough happens. The surfactant flood, with the highest mobility ratio, achieves breakthrough earlier than all the other floods, and also produces the highest fraction of water for almost the entire time. This can be attributed to the dual effect of the surfactant. It increases the relative permeabilities and reduces the capillary pressure barrier, 
thus facilitating the fluid flow. It can also be seen that the SP flood with the lower mobility ratio $\left(M_{\lambda}=2.35\right)$ always has a lower water cut in comparison to the SP flood with a higher mobility ratio $\left(M_{\lambda}=2.98\right)$, which supports the trend in their relative oil recovery efficiencies as well.

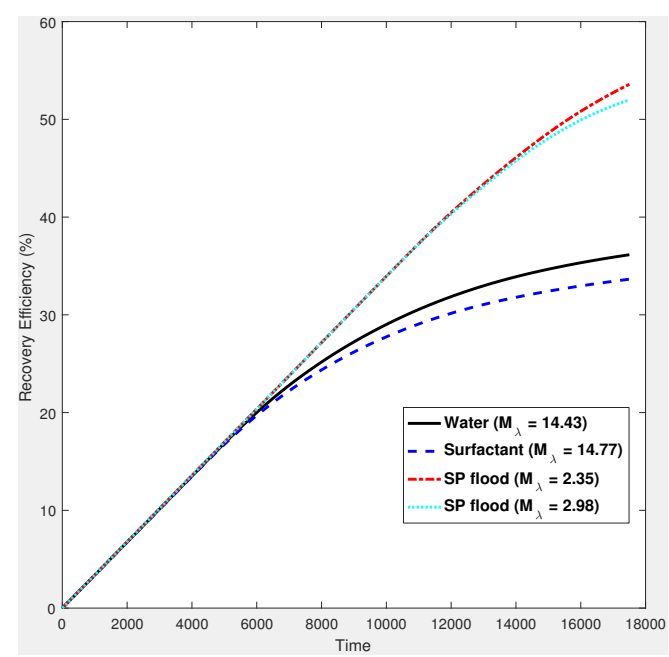

(a) Recovery efficiency versus time

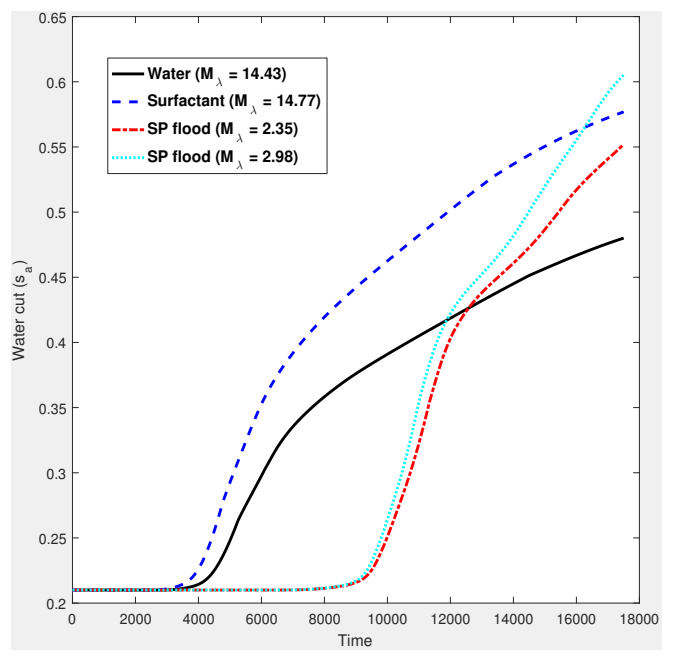

(b) Water cut versus time

Figure 26: Effect of the different flooding schemes on oil recovery efficiency and water cut in a quarter five-spot geometry with a heterogeneous permeability field given by Fig. 23 .

In Fig. 27, the second set of simulations is presented which compares water saturation surface plots between four different types of flooding schemes at three different time levels. The first column represents waterflooding $\left(M_{\lambda}=2.89, \Gamma_{0}=0\right)$, the second column represents surfactant flooding $\left(M_{\lambda}=3.04, \Gamma_{0}=0.1\right)$, the third and fourth columns represent two different configurations of SP flooding $\left(M_{\lambda}=1.91, \Gamma_{0}=0.01\right.$ and $M_{\lambda}=1.92, \Gamma_{0}=0.1$ ). In Fig. 24 we study the effect of polymer by changing the mobility ratios while keeping $\Gamma_{0}$ fixed. Here, we study the effect of surfactant by varying $\Gamma_{0}$ between the two SP floods. In Fig. 28 we compare the surfactant concentration surface plots for three floods. Specifically, the first column represents a surfactant flood $\left(M_{\lambda}=3.04, \Gamma_{0}=0.01\right)$ while the second and the third columns show the surfactant concentration plots during the two SP floods.

As observed in Fig. 27, the surfactant flood and both the SP floods perform better than the waterflood in recovering oil from the reservoir. This is reflected in the saturation surface plots of all the three floods where the region of higher saturation values (shown in the darker shade of red) have advanced further than that in the waterflood at comparable time levels. Even at water breakthrough, this is clearly visible. The other striking observation is that the surfactant flood and the two SP floods take somewhat comparable times while the waterflood takes significantly longer for water breakthrough. The surfactant flood is expected to achieve a quicker breakthrough as it has a higher mobility ratio than the waterflood, but the two SP floods achieve breakthrough earlier in spite of having lower mobility ratios than waterflood. This is because the surfactant mobilizes a larger percentage of the resident oil (by lowering capillary pressure and increasing relative permeabilities) and hence in Fig. 27, the interaction of the heterogeneity field used and the flood configuration allows the water saturation surfaces with higher values $\left(s_{a} \geq 0.5\right)$ to breakthrough earlier than in the waterflood. This kind of flow behavior is possible partly because the difference in mobility ratios between the floods in Fig. 27 is not very high.

The addition of polymer arrests the progress of the displacing phase along the heterogeneity induced "fingers" and this is visible near the advancing fronts (i.e. along the edges of the surface plots) if we compare the surfactant flood figures with any of the two SP flood figures. It is also confirmed by comparing the surfactant concentration surface plots, given in Fig. 28, especially at $t=6000$ and at water breakthrough. On 
the other hand, addition of surfactant while keeping the amount of polymer fixed does not have a significant impact, as seen in the last two columns of Fig. 27. The saturation surface plots are almost indistinguishable and these two floods also take very similar times to reach water breakthrough. Similarly, the second and the third columns of Fig. 28 show that the surfactant concentration surface plots for these two SP floods are almost the same indicating that they depend weakly on the concentration of the surfactant for this particular simulation. Overall, in the last two sets of simulations we observe that, even in the presence of both polymer and surfactant, the method is able to produce qualitatively accurate water saturation, polymer concentration and surfactant concentration plots through the heterogeneous permeability field while capturing the intricate details of the advancing fronts.

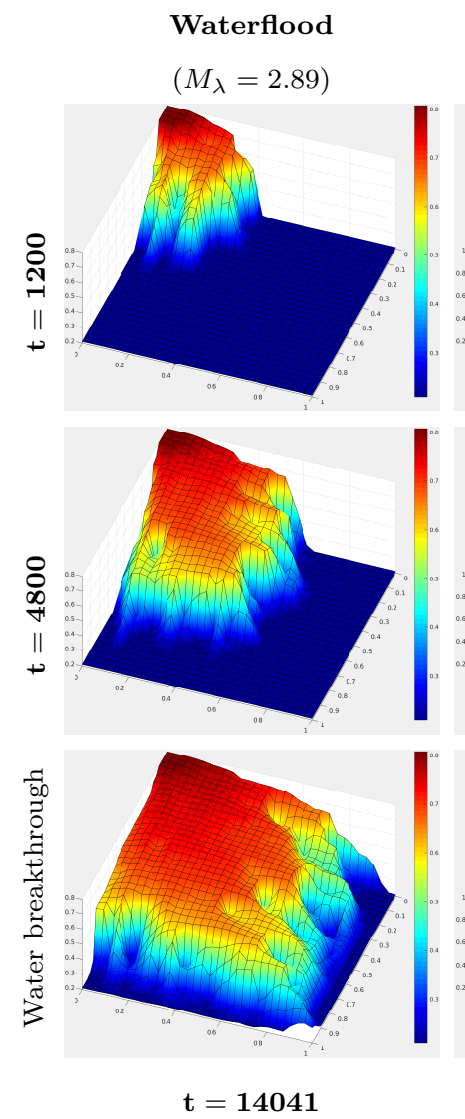

\section{Surfactant flood}

$\left(M_{\lambda}=3.04, \Gamma_{0}=0.1\right)$
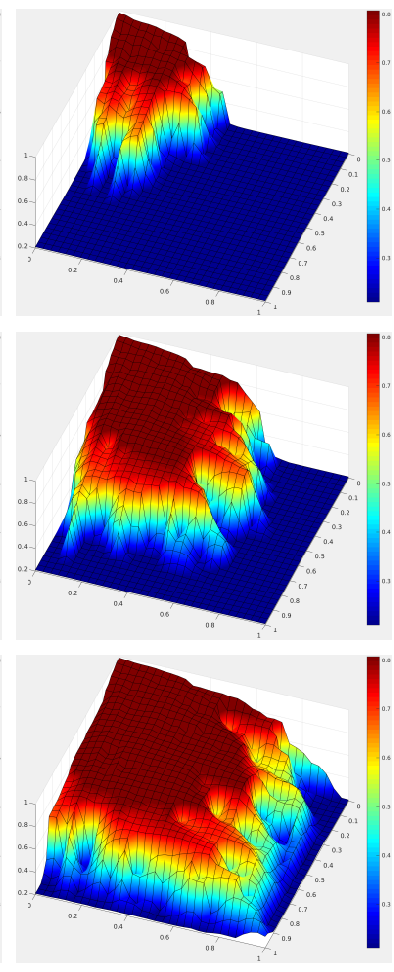

$\mathbf{t}=\mathbf{1 0 7 9 5}$
SP flood

$\left(M_{\lambda}=1.91, \Gamma_{0}=0.01\right)$
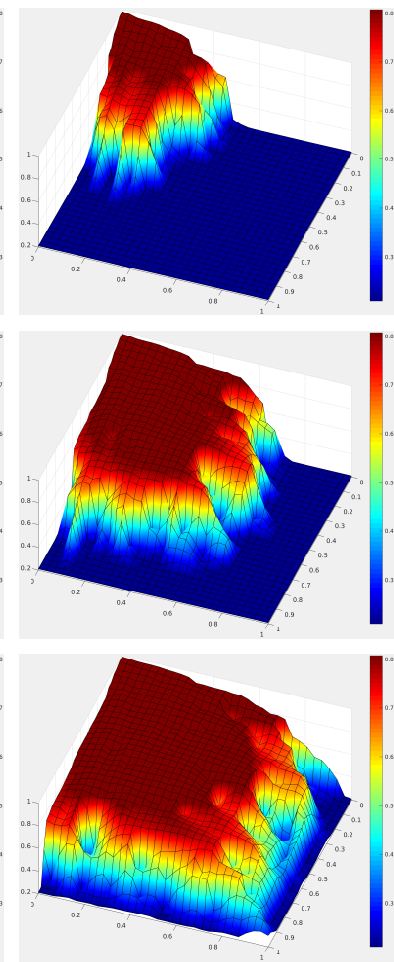

$\mathbf{t}=\mathbf{1 0 2 4 4}$
SP flood
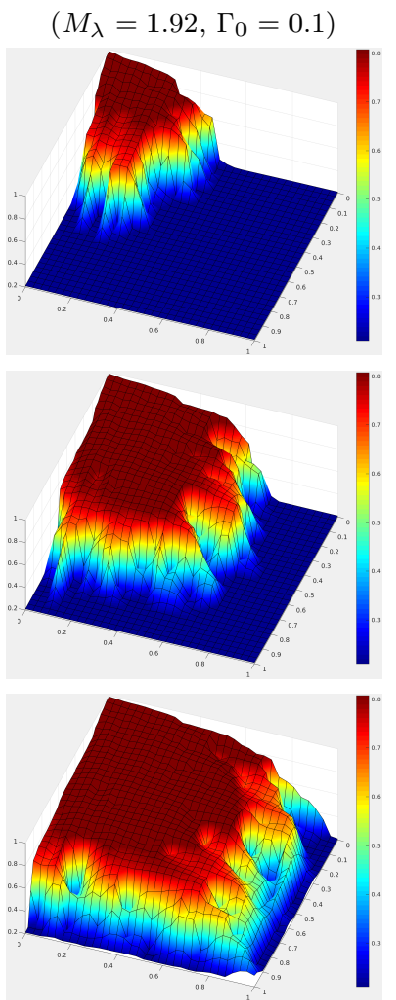

$\mathbf{t}=\mathbf{1 0 2 7 5}$

Figure 27: Comparison of water saturation surface plots in a quarter five-spot reservoir with spatial resolution of $32 \times 32$ during four different floods - waterflood, surfactant flood and two SP floods. The mobility ratio for the four floods were $M_{\lambda}=2.89,3.04,1.91,1.92 . \Gamma_{0}=0.1$ for the surfactant flood (second column) and the second SP flood (fourth column) and $\Gamma_{0}=0.01$ for the first SP flood (third column). The permeability field used is given in Fig. 23. The surface plots are shown at three different time levels including the plots at water breakthrough for each flood in the last row. The respective times at which water breakthrough happens for each flood is given below the last row of plots.

\subsubsection{Surfactant-polymer flooding in a rectilinear geometry}

In the final simulation, we compare four different types of floods in a rectilinear geometry with a spatial resolution of $60 \times 60$. These are waterflood $\left(M_{\lambda}=14.43, \Gamma_{0}=0\right)$, polymer flood $\left(M_{\lambda}=2.60, \Gamma_{0}=\right.$ 

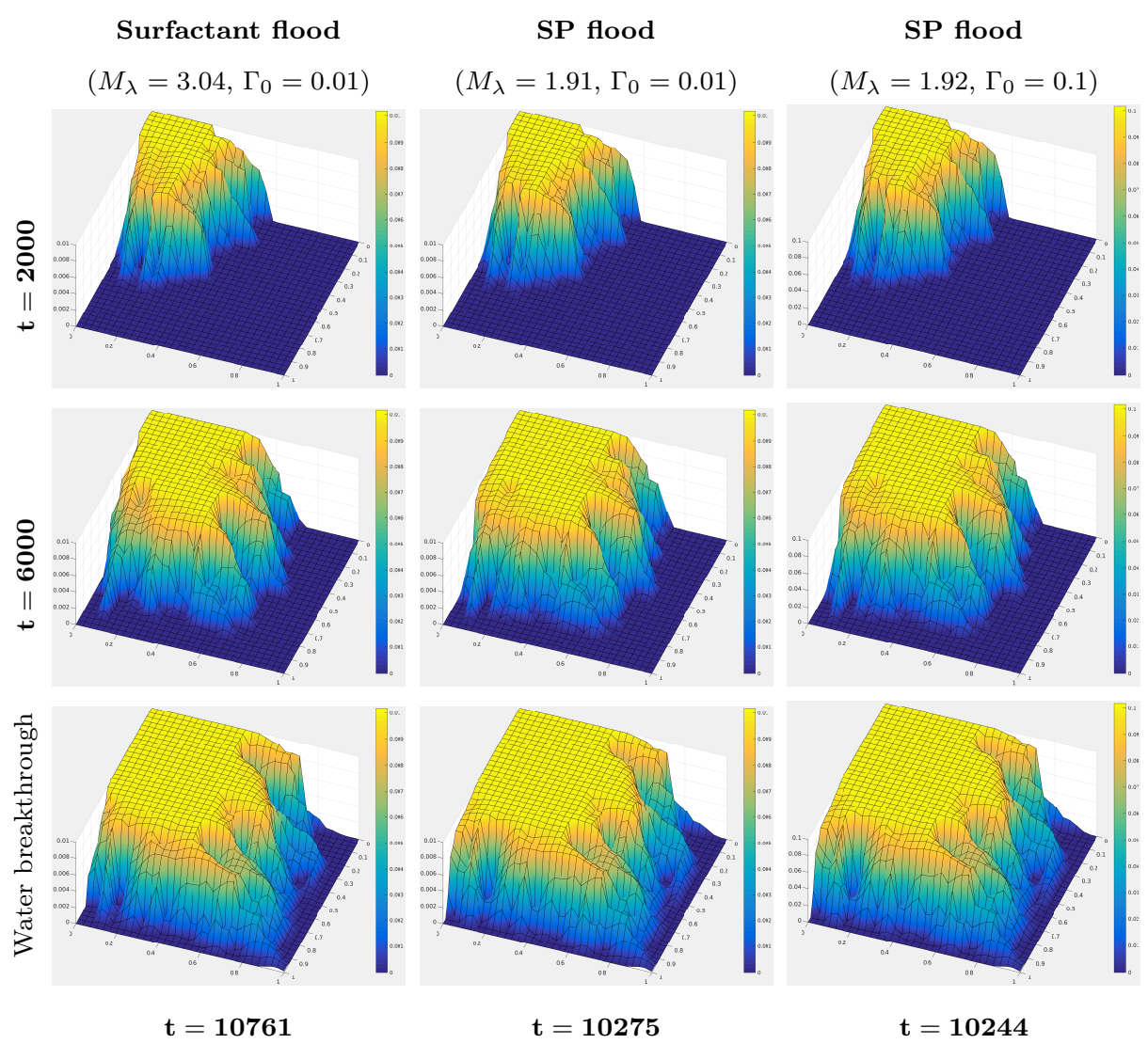

Figure 28: Comparison of surfactant concentration surface plots in a quarter five-spot reservoir with spatial resolution of $32 \times 32$ during three different floods. The mobility ratio was $M_{\lambda}=3.04$ for the surfactant flood (Column 1) and $M_{\lambda}=1.91,1.92$ for the two SP floods (Columns 2 and 3). $\Gamma_{0}=0.01$ for the first two columns and $\Gamma_{0}=0.1$ for the third column. The permeability field given in Fig. 23 has been used. The surface plots are shown at three different time levels including the plots at water breakthrough for each flood in the last row. The respective times at which water breakthrough happens for each flood is given below the last row of plots.

$0)$, surfactant flood $\left(M_{\lambda}=15.27, \Gamma_{0}=0.05\right)$ and SP flood $\left(M_{\lambda}=3.06, \Gamma_{0}=0.05\right)$. The heterogeneous permeability field given by eq. (63) has been used. Fig. 29 shows the water saturation contours at three different time levels. Fig. 30(a) shows the oil recovery efficiency with time for all the four floods and Fig. 30(b) shows the water recovery efficiency versus time.

The first important point to notice is the difference in time required to achieve water breakthrough for each of these floods. These time levels are provided below each of the water breakthrough contours in Fig. 29. The addition of polymer increases the viscosity of the displacing aqueous phase making it slower. For the purpose of demonstration, a very large drop in the mobility ratio from $M_{\lambda}=14.43,15.27$ (waterflood and the surfactant flood) to $M_{\lambda}=2.60,3.06$ (polymer and the SP flood) has been used to capture this effect. Hence the polymer flood takes the longest time to achieve water breakthrough as the polymer laden displacing phase becomes highly viscous. The addition of surfactant has the twin effect of lowering the capillary pressure barrier and increasing the relative permeability for the displacing phase and hence this makes it easier for the displacing phase to penetrate into the reservoir. In the SP flood, the dual effect of polymer and surfactant compete with each other and hence this flood achieves water breakthrough earlier than the polymer flood. However, for the particular setup chosen in this experiment, the surfactant in the system cannot completely 
overcome the slowdown caused by the polymer and hence pure waterflooding achieves water breakthrough earlier than both the polymer and the SP flooding. As expected intuitively, the surfactant flooding is the fastest. This is because the aqueous solution of surfactant is more mobile compared to pure water.

The effect of polymer in stabilizing the oil-water interface and thus reducing the growth of fingers can be seen as well in Fig. 29. From the first two rows in Fig. 29, it is evident that the growth of fingers slows down the most in the polymer flood, followed by the SP flood, the waterflood and the surfactant flood, at the same time level. This in turn ensures that by the time water breaks through a polymer flood achieves an improved sweep compared to the waterflood. As seen in the first two columns of the last row of Fig. 29, at water breakthrough the contours with higher levels of water saturation (shown in yellow and red) are trailing in the case of the waterflood to those of the polymer flood. In Fig. 30(a), the oil recovery curves for the waterflood and the polymer flood also support this observation. The oil recovery efficiency of the polymer flood is more than that of the waterflood and hence the water recovery efficiency of the polymer flood is lower as well (in Fig. 30). The water breakthrough times of all the floods can be clearly identified from the points in Fig. 30 where the curves abruptly depart from their steady coincidental trajectory. These times are also in agreement with the data obtained from the water saturation contour plots given in Fig. 29.

The effect of surfactant can be observed in the surfactant flood and the SP flood simulations shown in the last two columns of Fig. 29. The surfactant flood seems to enhance the finger formation, as can be seen by comparing the first and the third columns of Fig. 29 where the fingers advance faster in surfactant flood. The surfactant flood also has a lower oil recovery efficiency than the waterflood (see Fig. 30(a)). This result is not surprising if we compare the mobility ratios for each of the two floods. The surfactant flood has a higher mobility ratio than the waterflood and hence has a lower oil recovery efficiency. This is also supported by the water recovery curves of the waterflood and the surfactant flood in Fig. 30(b). The SP flood on the other hand combines the twin effect of the polymer and the surfactant and hence not only are the growth of fingers arrested, but also an improved sweep of the reservoir is achieved in comparison to the waterflood and the surfactant flood. Also, in Fig. 30(a), it can be seen that oil recovery curve for the SP flood steadily surpasses that of the surfactant flood, but trails that of the polymer flood. While this might look counterintuitive, a comparison of the mobility ratios of the polymer flood $\left(M_{\lambda}=2.60\right)$ and the SP flood $\left(M_{\lambda}=3.06\right)$ explains this phenomenon. From the standpoint of physics, this can be explained as well. The polymer flood has a lower mobility ratio and hence is able to achieve a better areal sweep of the reservoir. This means that the finger formations slowly become thicker and the resident oil in a larger area is swept through. Also, with the passage of time after water breakthrough, the region where the finger tips intersect with the line sink becomes larger compared to the SP flood, allowing for more discharge of oil. On the other hand, the surfactant in the SP flood tries to facilitate the movement of the more mobile displacing phase through the pathways formed by the finger formations. Hence, even though a larger percentage of oil is made available for recovery (in the SP flood), this is recovered only at the narrow tips where the fingers intersect with the line sink (see Fig. 29). The oil in the regions not yet swept by the displacing phase is not recovered. The better oil recovery efficiency of the polymer flood over the SP flood can be attributed to a combination of all these effects. The higher water recovery efficiency of the SP flood given in Fig. 30(b), compared to the polymer flood, supports these observations. Overall, it appears from our simulations that a careful study of the mobility ratios and the reservoir properties are critical for designing an efficient flooding scheme that can maximize recovery.

\section{Conclusions}

We have proposed a mathematical model of immiscible two-phase, multi-component, incompressible flow in porous media that arises in the context of chemical EOR processes, in particular polymer flooding and surfactant-polymer flooding. The mathematical model is a coupled nonlinear system of an elliptic equation for pressure and transport equations for water saturation, polymer concentration and surfactant concentration. This model takes into account the capillary pressure. A new global pressure formulation has been developed which renders the form of the elliptic equation with a discontinuous coefficient to be the same as the one 
without the capillary pressure included in the model, except for the fact that now we solve the elliptic equation for the global pressure. In this global pressure formulation, the system of flow and transport equations bears some similarity with that of a miscible displacement process which is known to be much simpler to solve numerically. A new hybrid numerical method based on an extended finite element method for the discontinuous coefficient elliptic global pressure equation and a modified method of characteristics in combination with a time-implicit finite difference method for the transport equations has been developed and implemented. Numerical simulations of several water, polymer and SP floods through heterogeneous porous media have been performed. Numerical results show the convergence and robustness of the numerical method both qualitatively and quantitatively.

The numerical method has been validated with an exact two dimensional solution for waterflooding. The corresponding error norms and order of accuracy calculations have been presented to demonstrate the accuracy of the method. Several other numerical simulations have been carried out on both a quarter five-spot geometry as well as a rectilinear geometry with four different types of heterogeneous permeability fields piecewise discontinuous with rectangular inclusions, the Upper-Ness formation type and the Tarbert formation type from the SPE10 dataset and a scalar, log-normal permeability field generated using a stationary,
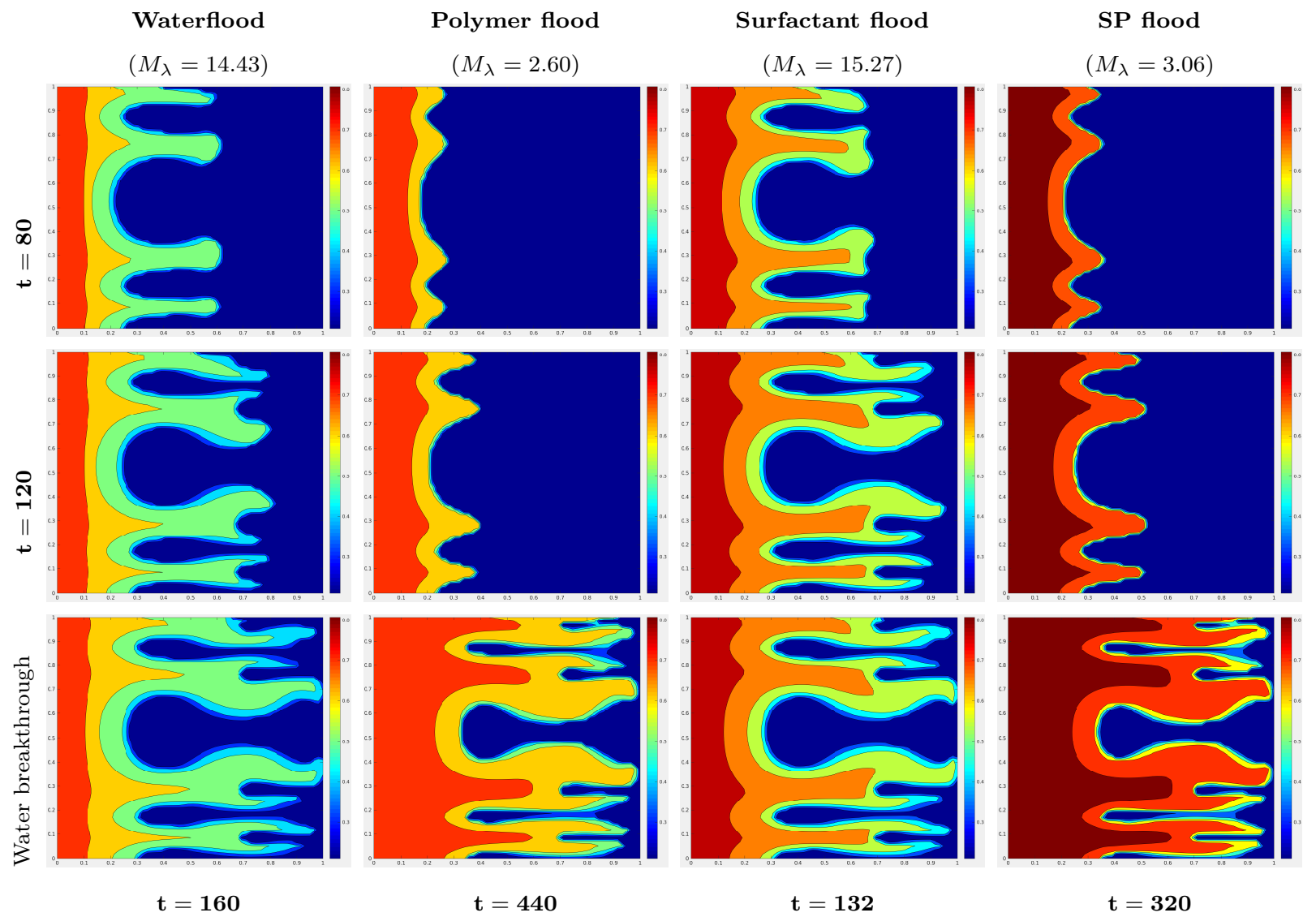

Figure 29: Comparison of saturation contours during four different types of chemical floods in a rectilinear geometry with $60 \times 60$ spatial resolution at three different time levels. The heterogeneous permeability field from [74] (plotted leftmost in Fig. 15) has been used. The saturations contours have been presented at $t=80$ (first row), $t=120$ (second row) and at water breakthrough in each of the different floods, with the corresponding time levels mentioned below each figure. 


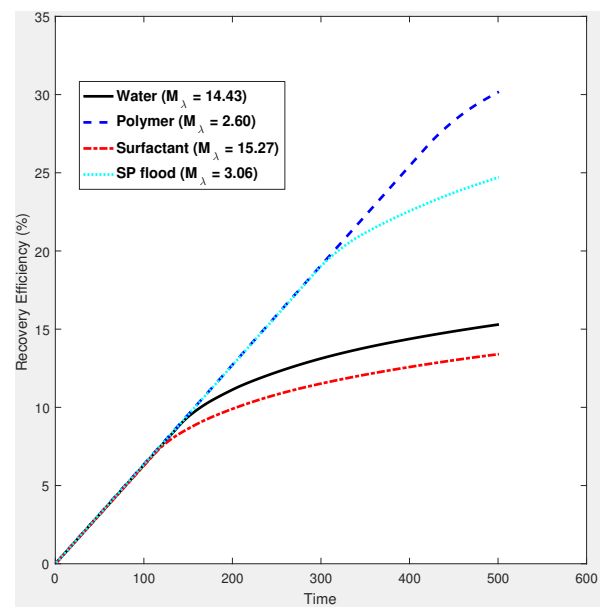

(a) Oil recovery versus time

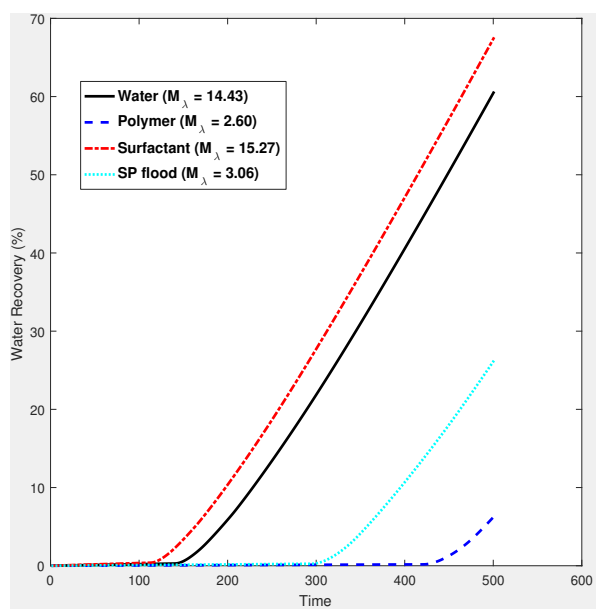

(b) Water cut versus time

Figure 30: Effect of the different flooding systems on oil recovery and water cut in a rectilinear geometry with a heterogeneous permeability field from [74] (plotted leftmost in Fig. 15).

isotropic, fractal Gaussian distribution. Two types of piecewise discontinuous permeability fields with one or more square inclusions have been used in the simulations. The method has been validated qualitatively by comparing these numerical results with those available in the literature. Simulations of a five-spot flood have been performed on a parallel and a diagonal grid to demonstrate that the method does not suffer from any serious grid orientation effects. The method is able to produce results which show that under similar reservoir conditions with heterogeneous Upper-Ness type permeability field data from the SPE10 benchmark problem, polymer flood improves the sweeping efficiency and inhibits growth of fingering instability in comparison to secondary recovery (waterflood). Numerical convergence of the method under grid refinement has been shown by performing polymer flood simulations in heterogeneous porous media using three different mesh sizes. The robustness of the method has been further demonstrated by simulating surfactant-polymer floods with various different compositions of the surfactant-polymer solution. The method is able to capture, fairly well, all the intricate details of the flow including the heterogeneity and viscosity driven finger formations. Using the saturation plots, the oil recovery curves and the water cut curves we are able to compare the relative effectiveness of different types of chemical flooding schemes in both types of flow geometry.

\section{Acknowledgment}

The work presented in this paper has been supported in part by the NPRP grant 08-777-1-141 through Qatar National Research Fund and by the U.S. National Science Foundation grant DMS-1522782. It is a pleasure to thank Liqun Wang, an ex-postdoc of P.D. for his participation at very early stages of the work on parts of the elliptic solver during 2011. The authors are also grateful to the reviewers for their helpful feedback and constructive suggestions that have helped us to improve this work significantly. Some of the numerical simulations were performed using high performance research computing resources provided by Texas A\&M University (http://hprc.tamu.edu). 


\section{References}

[1] P. Daripa, J. Glimm, B. Lindquist, M. Maesumi, O. McBryan. On the simulation of heterogeneous petroleum reservoirs. In: Numerical Simulation in Oil Recovery, IMA Vol. Math. Appl. 11, Springer, New York, NY, 1988, pp. 89-103.

[2] O. Karazincir, S. Thach, W. Wei, G. Prukop, T. Malik, V. Dwarakanath. Scale formation prevention during ASP flooding. In: SPE International Symposium on Oilfield Chemistry. Society of Petroleum Engineers, 2011.

[3] E. P. Katsanis, P. H. Krumrine, J. S. Falcone Jr. Chemistry of precipitation and scale formation in geological systems. In: SPE Oilfield and Geothermal Chemistry Symposium. Society of Petroleum Engineers, 1983.

[4] A. M. Alsofi, J. S. Liu, M. Han. Numerical simulation of surfactant-polymer coreflooding experiments for carbonates. J. Petrol. Sci. Eng., 111 (2013) 184-196.

[5] C. Gao, J. Shi, F. Zhao. Successful polymer flooding and surfactant-polymer flooding projects at Shengli oilfield from 1992 to 2012. J. Petrol. Explor. Prod. Technol., 4(1) (2014) 1-8.

[6] Z. Wu, X. Yue, T. Cheng, J. Yu, H. Yang. Effect of viscosity and interfacial tension of surfactantpolymer flooding on oil recovery in high-temperature and high-salinity reservoirs. J. Petrol. Explor. Prod. Technol., 4(1) (2014) 9-16.

[7] Q. Yu, H. Jiang, C. Zhao. Study of interfacial tension between oil and surfactant polymer flooding. Petrol. Sci. Technol., 28(18) (2010) 1846-1854.

[8] Y. Zhu, Y. Zhang, J. Niu, W. Liu, Q. Hou. The research progress in the alkali-free surfactant-polymer combination flooding technique. Petrol. Explor. Dev., 39(3) (2012) 371-376.

[9] R. Helmig. Multiphase Flow and Transport Processes in the Subsurface, fourth ed., Springer-Verlag Berlin Heidelberg, 1997.

[10] G. Chavent, J. Jaffré. Mathematical Models and Finite Elements for Reservoir Simulation, Volume 17, first ed., Studies in Mathematics and its applications, Amsterdam, North Holland, 1986.

[11] Z. Chen, R. E. Ewing. From single-phase to compositional flow: Applicability of mixed finite elements. Transport Porous Med., 27 (1997) 225-242.

[12] B. Amaziane, M. Jurak. A new formulation of immiscible compressible two-phase flow in porous media. CR Mec., 336(7) (2008) 600-605.

[13] B. Amaziane, M. Jurak, A. Z. Keko. Modeling and numerical simulations of immiscible compressible two-phase flow in porous media by the concept of global pressure. Transport Porous Med., 84(1) (2010) $133-152$.

[14] B. Amaziane, M. Jurak, A. Z. Keko. An existence result for a coupled system modeling a fully equivalent global pressure formulation for immiscible compressible two-phase flow in porous media. J. Differ. Equations, 250(3) (2011) 1685-1718.

[15] B. Amaziane, M. Jurak, A. Z. Keko. Numerical simulations of water-gas flow in heterogeneous porous media with discontinuous capillary pressures by the concept of global pressure. J. Comput. Appl. Math., 236(17) (2012) 4227-4244.

[16] B. Amaziane, M. Jurak, A. Z. Keko. Modeling compositional compressible two-phase flow in porous media by the concept of the global pressure. Computat. Geosci., 18(3-4) (2014) 297-309. 
[17] G. Chavent. A fully equivalent global pressure formulation for three-phases compressible flows. Appl. Anal., 88(10-11) (2009) 1527-1541.

[18] R. di Chiara Roupert, G. Chavent, G. Schäfer. Three-phase compressible flow in porous media: Total differential compatible interpolation of relative permeabilities. J. Comput. Phys., 229(12) (2010) 4762 4780 .

[19] P. Daripa, J. Glimm, J. Grove, B. Lindquist, O. McBryan. Reservoir simulation by the method of front tracking. In: Proc. of the IFE/SSI seminar on Reservoir Description and Simulation with Emphasis on EOR, 1986, pp. 1-18.

[20] P. Daripa, J. Glimm, B. Lindquist, O. McBryan. Polymer floods: A case study of nonlinear wave analysis and of instability control in tertiary oil recovery. SIAM J. Appl. Math., 48 (1988) 353-373.

[21] M. R. Islam, A. Chakma. Mathematical modelling of enhanced oil recovery by alkali solutions in the presence of cosurfactant and polymer. J. Petrol. Sci. Eng., 5(2) (1991) 105-126.

[22] Y. Shiyi, Y. Puhua, D. Zhongqiu, S. Kuiyou. Numerical simulation of alkali/surfactant/polymer flooding. In: International Meeting on Petroleum Engineering, 1995, pp. 139-152.

[23] L. J. Durlofsky. A triangle based mixed finite element-finite volume technique for modeling two phase flow through porous media. J. Comput. Phys., 105(2) (1993) 252-266.

[24] R. W. Healy, T. F. Russell. A finite-volume Eulerian-Lagrangian localized adjoint method for solution of the advection-dispersion equation. Water Resour. Res., 29(7) (1993) 2399-2413.

[25] Z. Chen, G. Huan, H. Wang. Computer simulation of compositional flow using unstructured control volume finite element methods. Computing, 78(1) (2006) 31-53.

[26] S. A. Sadrnejad, H. Ghasemzadeh, S. A. Ghoreishian Amiri, G. H. Montazeri. A control volume based finite element method for simulating incompressible two-phase flow in heterogeneous porous media and its application to reservoir engineering. Petrol. Sc., 9(4) (2012) 485-497.

[27] W. H. Reed, T. R. Hill. Triangular mesh methods for the neutron transport equation. Technical report, Los Alamos Scientific Lab., N.Mex. (USA), 1973.

[28] B. Rivière, M. F. Wheeler. Discontinuous Galerkin methods for flow and transport problems in porous media. Commun. Numer. Meth. Eng., 18(1) (2002) 63-68.

[29] P. Bastian. Higher Order Discontinuous Galerkin Methods for Flow and Transport in Porous Media, In: E. Bänsch (Ed.), Challenges in Scientific Computing - CISC 2002, Lecture Notes in Computational Science and Engineering, vol. 35, Springer Berlin Heidelberg, 2003, pp. 1-22.

[30] Y. Epshteyn, B. Rivière. Fully implicit discontinuous finite element methods for two-phase flow. Appl. Numer. Math., 57(4) (2007) 383-401.

[31] D. Nayagum, G. Schäfer, R. Mosé. Modelling two-phase incompressible flow in porous media using mixed hybrid and discontinuous finite elements. Computat. Geosci., 8(1) (2004) 49-73.

[32] H. Hoteit, A. Firoozabadi. Numerical modeling of two-phase flow in heterogeneous permeable media with different capillarity pressures. Adv. Water Resour., 31(1) (2008) 56-73.

[33] H. Hoteit, A. Firoozabadi. Multicomponent fluid flow by discontinuous Galerkin and mixed methods in unfractured and fractured media. Water Resour. Res., 41(11) (2005).

[34] J. Erhel, J.-R. de Dreuzy, B. Poirriez. Flow simulation in three-dimensional discrete fracture networks. SIAM J. Sci. Comput., 31(4) (2009) 2688-2705. 
[35] R. Ahmed, M. G. Edwards, S. Lamine, B. A. H. Huisman, M. Pal. Control-volume distributed multipoint flux approximation coupled with a lower-dimensional fracture model. J. Comput. Phys., 284 (2015) 462-489.

[36] Y. Efendiev, V. Ginting, T. Hou, R. Ewing. Accurate multiscale finite element methods for two phase flow simulations. J. Comput. Phys., 220 (2006) 155-174.

[37] E. Weinan, B. Engquist, X. Li, W. Ren. The heterogeneous multiscale method: A review. Commun. Comput. Phys., 2(3) (2007) 367-450.

[38] H. Zhou, S. H. Lee, H. A. Tchelepi. Multiscale finite volume formulation for the saturation equations. Soc. Petrol. Eng. J., 17 (2012) 198-211.

[39] Z. Chen. Characteristic-nonconforming finite-element methods for advection-dominated diffusion problems. Comput. Math. Appl., 48 (2004) 1087-1100.

[40] H. Wang, R. E. Ewing, T. F. Russell. Eulerian-Lagrangian localized adjoint methods for convectiondiffusion equations and their convergence analysis. IMA J. Numer. Anal., 15(3) (1995) 405-459.

[41] T. F. Russell, M. A. Celia. An overview of research on Eulerian-Lagrangian localized adjoint methods (ELLAM). Adv. Water Resour., 25(8) (2002) 1215-1231.

[42] J. Douglas Jr., F. Furtado, F. Pereira. On the numerical simulation of waterflooding of heterogeneous petroleum reservoirs. Comput. Geosc., 1 (1997) 155-190.

[43] H. Wang, R. E. Ewing, G. Qin, S. L. Lyons, M. Al-Lawatia, S. Man. A family of Eulerian-Lagrangian Localized Adjoint Methods for multi-dimensional advection-reaction equations. J. Comput. Phys., 152(1) (1999) 120-163.

[44] J. Douglas Jr., D. Frías, N. Henderson, F. Pereira. Simulation of single-phase multicomponent flow problems in gas reservoirs by Eulerian-Lagrangian techniques. Transport Porous Med., 50(3) (2003) 307-342.

[45] J. Douglas Jr., T. F. Russell. Numerical methods for convection-dominated diffusion problems based on combining the method of characteristics with finite element or finite difference procedures. SIAM J. Numer. Anal., 19(5) (1982) 871-885.

[46] H. K. Dahle, M. S. Espedal, R. E. Ewing, O. Sævereid. Characteristic adaptive subdomain methods for reservoir flow problems. Numer. Meth. Part. D. E., 6(4) (1990) 279-309.

[47] J. Douglas Jr., C. -S. Huang, F. Pereira. The modified method of characteristics with adjusted advection. Numer. Math., 83(3) (1999) 353-369.

[48] M. A. Celia, T. F. Russell, I. Herrera, R. E. Ewing. An Eulerian-Lagrangian localized adjoint method for the advection-diffusion equation. Adv. Water Resour., 13(4) (1990) 187-206.

[49] T. Arbogast, M. F. Wheeler. A characteristics-mixed finite element method for advection-dominated transport problems. SIAM J. Numer. Anal., 32(2) (1995) 404-424.

[50] J. Douglas Jr., F. Pereira, L. -M. Yeh. A locally conservative Eulerian-Lagrangian numerical method and its application to nonlinear transport in porous media. Computat. Geosci., 4(1) (2000) 1-40.

[51] F. Gao, Y. Yuan. The characteristic finite volume element method for the nonlinear convectiondominated diffusion problem. Comput. Math. Appl., 56(1) (2008) 71-81.

[52] K. Wang, H. Wang, M. Al-Lawatia. An Eulerian-Lagrangian discontinuous Galerkin method for transient advection-diffusion equations. Numer. Meth. Part. D. E., 23(6) (2007) 1343-1367. 
[53] M. S. Mahmood. Solution of nonlinear convection-diffusion problems by a conservative Galerkincharacteristics method. Numer. Math., 112(4) (2009) 601-636.

[54] C. L. Brown, G. A. Pope. Simulation of surfactant-enhanced aquifer remediation. Water Resour. Res., 30(11) (1994) 2959-2977.

[55] J. O. Amaefule, L. L. Handy. Effect of interfacial tensions on relative oil/water permeabilities of consolidated porous media. Soc. Petrol. Eng. J., 22(3) (1982) 371-381.

[56] Q. Liu, M. Dong, S. Ma, Y. Tu. Surfactant enhanced alkaline flooding for western Canadian heavy oil recovery. Colloid. Surface. A, 293(13) (2007) 63-71.

[57] R. H. Brooks, A. T. Corey. Properties of porous media affecting fluid flow. J. Irrig. Drain. Div., 92,(IR2) (1966) 61-90.

[58] A. T. Corey. Mechanics of Immiscible Fluids in Porous Media, second ed., Water Resources Publications, Littleton, Colorado, 1986.

[59] M. Th. van Genuchten. A closed form equation for predicting the hydraulic conductivity of unsaturated soils. Soil Sci. Soc. Am. J., 44 (1980) 892-898.

[60] J. C. Parker, R. J. Lenhard, T. Kuppusamy. A parametric model for constitutive properties governing multiphase flow in porous media. Water Resour. Res., 23(4) (1987) 618-624.

[61] B. Ghanbarian-Alavijeh, A. Liaghat, G. -H. Huang, M. Th. van Genuchten. Estimation of the van Genuchten Soil Water Retention Properties from Soil Textural Data. Pedosphere, 20(4) (2010) 456465.

[62] S. Hou, W. Wang, L. Wang. Numerical method for solving matrix coefficient elliptic equation with sharp-edged interfaces. J. Comput. Phys., 229 (2010) 7162-7179.

[63] Z. Li, T. Lin, X. Wu. New Cartesian grid methods for interface problems using the finite element formulation. Numer. Math., 96 (2003) 61-98.

[64] X. -D. Liu, R. P. Fedkiw, M. Kang. A boundary condition capturing method for Poisson's equation on irregular domains. J. Comput. Phys., 160 (2000) 151-178.

[65] J. Douglas Jr. Finite difference methods for two-phase incompressible flow in porous media. SIAM J. Numer. Anal., 20(4) (1983) 681-696.

[66] R. E. Ewing, H. Wang. A summary of numerical methods for time-dependent advection-dominated partial differential equations. J. Computat. Appl. Math., 128(1-2) (2001) 423-445.

[67] M. R. Borges, F. Pereira, H. P. Amaral Souto. Efficient generation of multi-scale random fields: A hierarchical approach. Int. J. .Numer. Method. Biomed. Eng., 26 (2010) 176-189.

[68] J. Glimm, W. B. Lindquist, F. Pereira, Q. Zhang. A theory of macrodispersion for the scale-up problem. Transport Porous Med., 13(1) (1993) 97-122.

[69] F. Furtado, F. Pereira. Crossover from nonlinearity controlled to heterogeneity controlled mixing in two-phase porous media flows. Computat. Geosci., 7(2) (2003) 115-135.

[70] E. Abreu. Numerical modelling of three-phase immiscible flow in heterogeneous porous media with gravitational effects. Math. Comput. Simulat., 97 (2014) 234-259.

[71] J. Li, B. Rivière. Numerical solutions of the incompressible miscible displacement equations in heterogeneous media. Comput. Method..Appl. M., 292 (2014) 107-121. 
[72] G. Chavent. A new formulation of diphasic incompressible flows in porous media. In: P. Germain, B. Nayroles (Eds.), Applications of Methods of Functional Analysis to Problems in Mechanics: Joint Symposium IUTAM/IMU held in Marseille, September 1-6, 1975, Springer Berlin Heidelberg, 1976, pp. 258-270.

[73] Y. C. Yortsos. The relationship between immiscible and miscible displacement in porous media. AIChE J., 33(11) (1987) 1912-1915.

[74] J. A. Ferreira, G. Pena, G. Romanazzi. Anomalous diffusion in porous media. Appl. Math. Model., 40 (2015) 1850-1862.

[75] C.-C. Chueh, N. Djilali, W. Bangerth. An h-adaptive operator splitting method for two-phase flow in 3D heterogeneous porous media. SIAM J. Sci. Comput., 35(1) (2013) 149-175.

[76] M. A. Christie, M. J. Blunt. Tenth SPE Comparative Solution Project: A comparison of upscaling techniques. SPE Reserv. Eval. Eng., 4(4) (2001) 308-317.

[77] M. R. Todd, P. M. O’Dell, G. J. Hirasaki. Methods for increased accuracy in numerical reservoir simulators. Soc. Petrol. Eng. J., 12(6) (1972) 515-530.

[78] J. Kozdon, M. Gerritsen, M. Christie. Grid orientation revisited: Near-well, early-time effects and solution coupling methods. Transport Porous Med., 73(3) (2008) 255-277.

[79] P. Daripa, G. Pasa. An optimal viscosity profile in enhanced oil recovery by polymer flooding. Int. J. Eng. Sci., 42(19-20) (2004) 2029-2039.

[80] P. Daripa, X. Ding. A numerical study of instability control for the design of an optimal policy of enhanced oil recovery by tertiary displacement processes. Transport Porous Med., 93(3) (2012) 675703.

[81] P. Daripa. Fluid dynamical and modeling issues of chemical flooding for enhanced oil recovery. In: ASME 2013 32nd International Conference on Ocean, Offshore and Arctic Engineering, Nantes, France, 2013, pp. 1-8. 